Convergência da videoeletroencefalografia prolongada e da ressonância magnética de encéfalo na determinação de zonas epileptogênicas extrahipocampais
presumidas.

Ribeirão Preto 



\title{
Convergência da videoeletroencefalografia prolongada e da ressonância magnética de encéfalo na determinação de zonas epileptogênicas extrahipocampais presumidas.
}

\author{
Tese apresentada ao Programa de Pós- \\ Graduação em Neurologia da Faculdade de \\ Medicina de Ribeirão Preto da Universidade \\ de São Paulo para obtenção do título de Dou- \\ tor em Ciências (Neurologia).
}

\author{
Universidade de São Paulo \\ Faculdade de Medicina de Ribeirão Preto \\ Departamento de Neurociências e Ciências do Comportamento
}

Orientador: Américo Ceiki Sakamoto

Ribeirão Preto

2016 
Autorizo a reprodução e divulgação total ou parcial deste trabalho, por qualquer meio convencional ou eletrônico, para fins de estudo e pesquisa, desde que citada a fonte.

\section{BRUNO ZANOTELLI MONNERAT}

Convergência da videoeletroencefalografia prolongada e da ressonância magnética de encéfalo na determinação de zonas epileptogênicas extrahipocampais presumidas./ BRUNO ZANOTELLI MONNERAT. - Ribeirão Preto, 2016-

136 p. : 8 il. (algumas color.) ; $30 \mathrm{~cm}$.

Orientador: Américo Ceiki Sakamoto

Tese (Doutorado) - Universidade de São Paulo

Faculdade de Medicina de Ribeirão Preto

Departamento de Neurociências e Ciências do Comportamento, 2016.

1. Epilepsia. 2. Displasia cortical. 3. Videoeletroencefalografia. 4. Ressonância magnética. 5. Cirurgia de epilepsia. 6. Acurácia I. Américo C Sakamoto. II. Universidade de São Paulo. III. Faculdade de Medicina de Ribeirão Preto. IV. Convergência da videoeletroencefalografia prolongada e da ressonância magnética de encéfalo na determinação de zonas epileptogênicas extrahipocampais presumidas 


\title{
Convergência da videoeletroencefalografia prolongada e da ressonância magnética de encéfalo na determinação de zonas epileptogênicas extrahipocampais presumidas.
}

Tese apresentada ao Programa de PósGraduação em Neurologia da Faculdade de Medicina de Ribeirão Preto da Universidade de São Paulo para obtenção do título de Doutor em Ciências (Neurologia).

Trabalho em análise. Ribeirão Preto, 20/07/2016:

\author{
Américo Ceiki Sakamoto \\ Orientador, FMRP-USP \\ Prof. Dr. Fernando Cendes \\ UNICAMP \\ Dr. Luís Otávio Sales Ferreira Caboclo \\ HIAE \\ Prof Dr. Antonio Carlos dos Santos \\ FMRP-USP \\ Dr. Tonicarlo Rodrigues Velasco \\ HCRP \\ Ribeirão Preto \\ 2016
}



Aos pacientes com epilepsia, pela sua cooperação e resiliência; aos funcionários do Centro de Cirurgia de Epilepsia (CIREP), por sua generosidade e trabalho extraordinário ao longo dos anos; e a todos aqueles que, como eu, continuam seus estudos em epilepsia. Não desanimemos jamais de compreender e melhorar as condições das pessoas que sofrem dessa doença. 



\section{Agradecimentos}

Ao Prof. Dr. Américo C Sakamoto, pela oportunidade e confiança;

Ao Dr. Tonicarlo R Velasco, pela colaboração e crítica;

Aos Drs. Ana Paula P Martins, Ana Hamad, Veriano Alexandre Jr, Frederico Nakano e Prof. João Leite, pelo apoio;

Às Sras. Adriana Nogueira, Elídia Magnani e Silvana Lo Turco, por deixarem as coisas mais fáceis.

Aos meus pais Ignácio e Luiza, e à minha irmã Paula, por tudo.

Leilane, outro beijo. 



\section{Resumo}

MONNERAT, B. Z. Convergência da videoeletroencefalografia prolongada e da ressonância magnética de encéfalo na determinação de zonas epileptogênicas extrahipocampais presumidas. 2016. 136 f. Tese (Doutorado) - Faculdade de Medicina de Ribeirão Preto, Universidade de São Paulo, Ribeirão Preto, 2016.

Pacientes com epilepsia farmacorresistente, frequentemente, possuem lesões extrahipocampais como etiologia. Muitas vezes, estes pacientes se beneficiam de lesionectomias para redução da ocorrência de crises epilépticas. Para que possam se submeter a este procedimento, atualmente é necessário o uso tanto da videoeletroencefalografia prolongada (VEEG) quanto da imagem de ressonância magnética do encéfalo (IRM) para delimitação apurada da zona epileptogênica, local que deve ser ressecado para controle das crises. No presente trabalho, foi estudada a acurácia diagnóstica da VEEG e da IRM na determinação da zona epileptogênica de pacientes com displasia cortical focal. Comparou-se os locais de ocorrência da zona de início ictal (VEEG) e da lesão epileptogênica (IRM) se concordantes ou discordantes com o local da cirurgia. Foram revisados os prontuários médicos de 209 pacientes, sendo o padrão de referência (local da cirurgia) e tempo de acompanhamento pós-operatório superior a 12 meses disponíveis em 43 pacientes. A VEEG apresentou sensibilidade de 85,7\% (IC 95\% 62,6-96,2) e especificidade de 41,1\% (IC 95\% 19,4-66,5), com valor preditivo positivo de 64,2\% (IC 95\% 44,1-80,6) e valor preditivo negativo de 70\% (IC 95\% 35,3-91,9). A IRM apresentou sensibilidade de 91,6\% (IC 95\% 71,5-98,5) e especificidade de $36,8 \%$ (IC 95\% 17,2-61,3), com valor preditivo positivo de $64,7 \%$ (IC $95 \%$ 46,4-79,6) e valor preditivo negativo de 77,7\% (IC 95\% 40,1-96). As diferenças de sensibilidade e especificidade, áreas sob as curvas ROC e os índices de Youden não foram significativas. A concordância dos resultados da VEEG e da IRM foi moderada ( $\mathrm{k}=0,599$; $\mathrm{p}<0,01 ;$ IC $95 \%$ 0,468-0,730).

Palavras-chaves: epilepsia. displasia cortical. videoeletroencefalografia. imagem de ressonância magnética. cirurgia de epilepsia. acurácia 



\section{Abstract}

MONNERAT, B. Z. The convergence of long-term videoelectroencefalography and brain magnetic resonance imaging in the delineation of presumed extrahippocampal epileptogenic zones. 2016. 136 f. Tese (Doutorado) - Faculdade de Medicina de Ribeirão Preto, Universidade de São Paulo, Ribeirão Preto, 2016.

Patients with drug-resistant epilepsy frequently have extrahippocampal lesions as etiology. A large proportion of these patients might benefit from lesionectomy for the reduction of seizures. For surgery to be undertaken, it is usually performed both long-term videoelectroencephalography monitoring (VEEG) and magnetic resonance imaging of the brain (MRI) for the precise delimitation of the epileptogenic zone, the region that must be resected for seizure control. In the present study, the diagnostic accuracy of VEEG and MRI were studied in the localization of the epileptogenic zone in patients with focal cortical dysplasia. The seizure-onset zone (VEEG) and the region of epileptogenic lesion (MRI) were compared whereas concordant or discordant regarding surgery region. Medical charts of 209 patients were reviewed, being the reference standard (surgery region) and post-surgical follow-up longer than 12 months available in 43 patients. Videoelectroencephalography has a sensitivity of $85.7 \%$ (95\% CI 62.6-96.2) and specificity of $41.1 \%$ (95\% CI 19.4-66.5), with positive predictive value of $64.2 \%$ (95\% CI 44.1-80.6) and negative predictive value of $70 \%$ (95\% CI 35.3-91.9). Magnetic resonance imaging has a sensitivity of $91.6 \%$ (95\% CI 71.5-98.5) and specificity of 36.8\% (95\% CI 17.2-61.3), with positive predictive value of $64.7 \%$ (95\% CI 46.4-79.6) and negative predictive value of $77.7 \%$ (95\% CI 40.1-96). The differences of sensitivity and specificity, areas under the ROC curves and Youden's indexes were not significant. The concordance between the results of VEEG and MRI was moderate $(\mathrm{k}=0.599 ; \mathrm{p}<0.01 ; 95 \%$ CI $0.468-0.730)$.

Key-words: epilepsy. cortical dysplasia. videoelectroencephalography. magnetic resonance imaging. epilepsy surgery. accuracy. 



\section{Lista de ilustrações}

Figura 1 - Imagem de ressonância magnética da displasia cortical focal, evidenciada por hipersinal na região temporal esquerda na sequência FLAIR (A,B) e hiposinal na região parietal direita na sequência T1 (C). Extraído de VELEZ-RUIZ; KLEIN, 2012. . . . . . . . . . . . . . . 30

Figura 2 - Instrumento para captação de dados do prontuário médico. . . . . . . . 46

Figura 3 - Fluxo da amostra. VEEG: vídeoeletroencefalografia; DCF: displasia cortical focal; IRM: imagem de ressonância magnética de encéfalo; CIREP: Centro de Cirurgia de Epilepsia - HC USP Ribeirão. . . . . . 52

Figura 4 - Distribuição das idades à VEEG e à cirurgia. . . . . . . . . . . . . . . 53

Figura 5 - Duração da epilepsia à VEEG e à cirurgia. . . . . . . . . . . . . . . . . 54

Figura 6 - Fluxo de resultados para cálculos de acurácia. DCF: displasia cortical focal; IRM: imagem de ressonância magnética; VEEG: videoeletroencefalografia. . . . . . . . . . . . . . . . 63

Figura 7 - Curvas ROC (receiver-operating characteristic). . . . . . . . . . . . 68 Figura 8 - Cálculo do poder estatístico do estudo. . . . . . . . . . . . . . 76 



\section{Lista de tabelas}

Tabela 1 - Distribuição da amostra pela tecnologia de IRM. . . . . . . . . . . . . 53

Tabela 2 - Distribuição da amostra pelo tipo de lesão à anatomia patológica. . . . 54

Tabela 3 - Distribuição da amostra pelo local da lesão epileptogênica (IRM). . . . 55

Tabela 4 - Distribuição da amostra pelo hemisfério da lesão epileptogênica (IRM). 55

Tabela 5 - Distribuição da amostra pela região da zona de início ictal (VEEG). . . 55

Tabela 6 - Distribuição da amostra pelo hemisfério da zona de início ictal (VEEG). 56

Tabela 7 - Localização da zona irritativa (EEG interictal). . . . . . . . . . . . . . 56

Tabela 8 - Lateralização da zona irritativa (EEG interictal). . . . . . . . . . . . 56

Tabela 9 - Localização da cirurgia. . . . . . . . . . . . . . . . . . 57

Tabela 10 - Realização de investigação invasiva. . . . . . . . . . . . . . . . . . 57

Tabela 11 - Lateralização dos eletrodos invasivos. . . . . . . . . . . . . . . . 57

Tabela 12 - Realização de estimulação cortical. . . . . . . . . . . . . . . . . 58

Tabela 13 - Duração do acompanhamento pós-operatório. . . . . . . . . . . . . . . 58

Tabela 14 - Desfecho pós-cirúrgico (>12 meses de acompanhamento). . . . . . . . 58

Tabela 15 - Concordância da zona de início ictal (VEEG) com a lesão epileptogênica (IRM). . . . . . . . . . . . . . . . 6 60

Tabela 16 - Concordância da zona de início ictal (VEEG) com o local da cirurgia. . 61

Tabela 17 - Concordância da lesão epileptogênica (IRM) com o local da cirurgia. $\quad 62$

Tabela 18 - Tabela cruzada modelo. . . . . . . . . . . . . . . . 63

Tabela 19 - Tabela cruzada para VEEG. . . . . . . . . . . . . . . . . . . . 64

Tabela 20 - Desempenho diagnóstico da VEEG. . . . . . . . . . . . . . . . . . . . 64

Tabela 21 - Tabela cruzada para IRM. . . . . . . . . . . . . . . . . . . . . 64

Tabela 22 - Desempenho diagnóstico da IRM. . . . . . . . . . . . . . . . . . . . 65

Tabela 23 - Comparação das sensibilidades dos dois métodos (pacientes livres de crises). . . . . . . . . . . . . . . . . 6 65

Tabela 24 - Comparação das especificidades dos dois métodos (pacientes não-livres de crises). . . . . . . . . . . . . . . . 66 66

Tabela 25 - Diferenças no desempenho diagnóstico da VEEG e da IRM. . . . . . . . 67

Tabela 26 - Áreas sob as curvas ROC. . . . . . . . . . . . . . . . . . . . 68

Tabela 27 - Coeficiente de correlação: diferença das áreas sob as curvas ROC. . . . 69

Tabela 28 -Índices de Youden. . . . . . . . . . . . . . . . . . 71 



\title{
Lista de abreviaturas e siglas
}

\author{
AUCROC Área sob a curva ROC \\ CEP Comitê de Ética em Pesquisa \\ DCF Displasia cortical focal \\ ECoG Eletrocorticografia intraoperatória \\ EEG Eletroencefalografia ambulatorial \\ FLAIR Fluid attenuation inversion recovery \\ IC Intervalo de confiança \\ ILAE Liga Internacional Contra Epilepsia \\ IRM Imagem de ressonância magnética de encéfalo \\ J I Indice de Youden \\ K Coeficiente Kappa de Cohen \\ PET Tomografia por emissão de pósitrons \\ $\mathrm{R} \$ \quad$ Reais \\ RNM Ressonância nuclear magnética \\ ROC Receiver operating characteristic \\ SAME Serviço de Arquivo Médico \\ SIGTAP Sistema de Gerenciamento da Tabela de Procedimentos, Medicamentos \\ e OPM do SUS \\ SPECT Tomografia computadorizada por emissão de fóton único \\ STARD Standards for Reporting of Diagnostic Accuracy Studies \\ SUS S Sistema Único de Saúde \\ T Tesla \\ US\$ Dólares americanos \\ VEEG Videoeletroencefalografia prolongada
}





\section{Sumário}

\begin{tabular}{|l|l|}
\hline INTRODUÇÃO & 23 \\
\hline
\end{tabular}

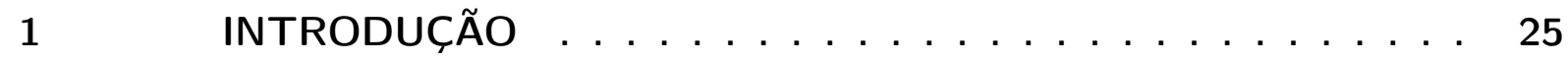

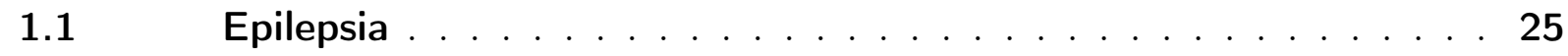

$1.1 .1 \quad$ Definições e impacto $\ldots \ldots \ldots \ldots \ldots \ldots . \ldots \ldots 25$

$1.1 .2 \quad$ Epidemiologia . . . . . . . . . . . . . . . . . . . . 26

$1.1 .3 \quad$ Classificações . . . . . . . . . . . . . . . . . . 26

$1.2 \quad$ Epilepsias lesionais extrahipocampais . . . . . . . . . . 27

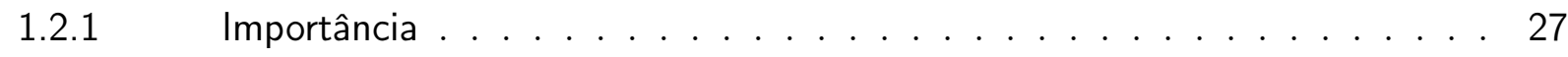

$1.2 .2 \quad$ Displasia cortical focal $\ldots \ldots \ldots \ldots \ldots \ldots$

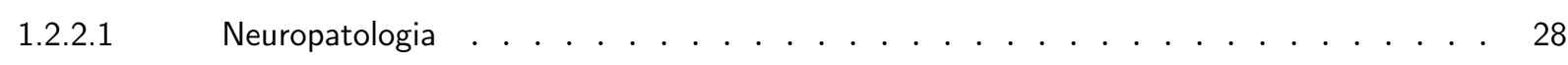

1.2 .2 .2 Apresentação clínica . . . . . . . . . . . . . . . . . . . . . . . . . . . . . 29

$1.2 .2 .3 \quad$ Apresentação radiológica . . . . . . . . . . . . . . . . . . . . . . . . . . 29

$1.3 \quad$ Estudos em técnicas diagnósticas $\ldots \ldots \ldots \ldots$

$1.3 .1 \quad$ Ressonância magnética de encéfalo . . . . . . . . . . . . . . 31

1.3 .11 Desenvolvimento $\ldots \ldots \ldots \ldots \ldots \ldots \ldots \ldots$

1.3 .1 .2 Fundamentos $\ldots \ldots \ldots \ldots \ldots \ldots$

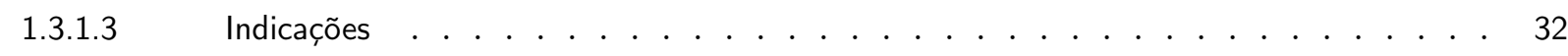

$1.3 .2 \quad$ Videoeletroencefalografia prolongada $\ldots \ldots \ldots \ldots 33$

$1.3 .2 .1 \quad$ Desenvolvimento $\ldots \ldots \ldots \ldots \ldots$

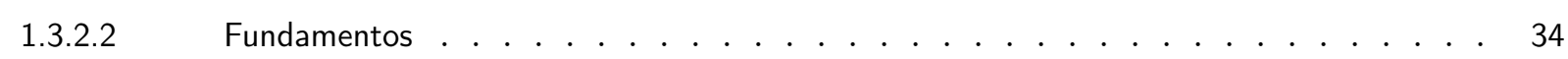

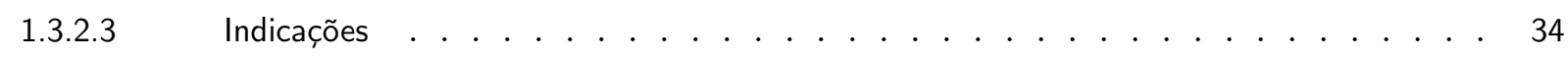

$1.4 \quad$ Cirurgia de epilepsia lesional extrahipocampal $\ldots \ldots \ldots 35$

1.4 .1 Desenvolvimento . . . . . . . . . . . . . . . . . . . . . . . . . 35

$1.4 .2 \quad$ Fundamentos $\ldots \ldots \ldots \ldots \ldots \ldots$

$1.4 .3 \quad$ Indicações . . . . . . . . . . . . . . . . . . . . . . 37

$1.5 \quad$ Perguntas do trabalho atual $\ldots \ldots \ldots \ldots \ldots$

2 OBJETIVOS . . . . . . . . . . . . . . . . . . . . 39

$2.1 \quad$ Objetivo principal $\ldots \ldots \ldots \ldots$

$2.2 \quad$ Objetivo secundário $\ldots \ldots \ldots . \ldots \ldots$

\begin{tabular}{lll}
\hline 11 & DESENVOLVIMENTO
\end{tabular}

3 PARTICIPANTES E MÉTODOS . . . . . . . . . . . . . . 43

$3.1 \quad$ Desenho do estudo . . . . . . . . . . . . . . . . . . . 43 
$3.1 .1 \quad$ Critérios de inclusão $\ldots \ldots \ldots$. . . . . . . . . . . . 43

$3.1 .2 \quad$ Critérios de exclusão . . . . . . . . . . . . . . . . . 43

3.2 Identificação da amostra . . . . . . . . . . . . . . . 44

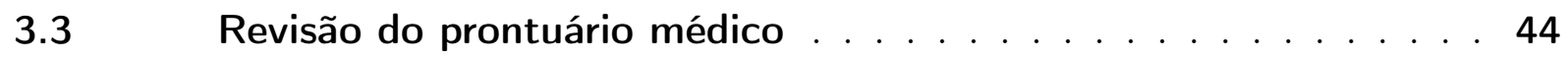

$3.4 \quad$ Análise estatística . . . . . . . . . . . . . . . . . . . . . . . . 47

$3.5 \quad$ Aprovação do comitê de ética em pesquisa . . . . . . . . . . 48

3.6 Declaração de conflitos de interesse . . . . . . . . . . . . 48

$3.7 \quad$ Custeio . . . . . . . . . . . . . . . . . . . . . . . . . . . . . . . . . 49

$4 \quad$ RESULTADOS . . . . . . . . . . . . . . . . . . . . . . . . . 51

$4.1 \quad$ Participantes do estudo . . . . . . . . . . . . . . 51

$4.2 \quad$ Dados descritivos . . . . . . . . . . . . . . . . . . . . 51

$4.3 \quad$ Análise da convergência entre a VEEG e a IRM . . . . . . . . 58

$4.4 \quad$ Análise da acurácia da VEEG e da IRM . . . . . . . . . . . . . . 59

$4.5 \quad$ Comparação da acurácia da VEEG e da IRM . . . . . . . . . . . 64

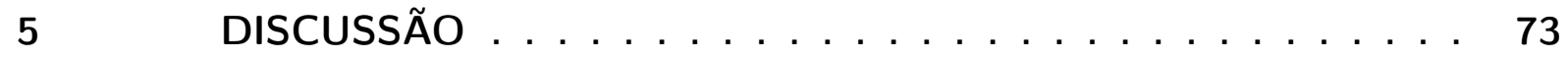

$5.1 \quad$ Interpretação dos resultados $\ldots \ldots \ldots \ldots 73$

$5.2 \quad$ Limitações $\ldots \ldots \ldots \ldots$. . . . . . . . . . . . . . . . . . . . . . . 75

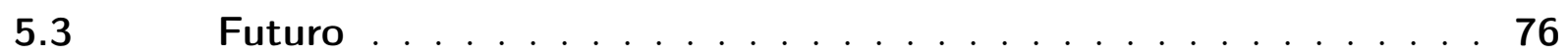

$5.4 \quad$ Aplicação $\ldots \ldots \ldots \ldots 77$

\begin{tabular}{lll}
\hline III CONCLUSÃO & 79
\end{tabular}

$6 \quad$ CONCLUSÃO $\ldots \ldots \ldots \ldots \ldots \ldots \ldots$

$6.1 \quad$ Conclusão principal $\ldots \ldots \ldots \ldots$. . . . . . . . . . 81

$6.2 \quad$ Conclusão secundária $\ldots \ldots \ldots \ldots 1$

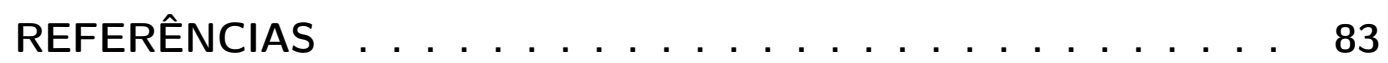

\begin{tabular}{ll}
\hline APÊNDICES & 91
\end{tabular}

APÊNDICE A - ÍNDICE PARA LOCALIZAÇÃO DE DADOS (INICIATIVA STARD). . . . . . . . . . . . 93

APÊNDICE B - ARTIGO SUBMETIDO AO PERIÓDICO SEIZURE . . . . . . . . . . . . . . 95 
ANEXO A - APROVAÇÃO DO COMITÊ DE ÉTICA EM PESQUISA (CEP). . . . . . . . . . . . . 133 

Parte I

Introdução 



\section{Introdução}

\subsection{Epilepsia}

A cirurgia para epilepsia lesional (aquela em que há substrato estrutural identificado à imagem de ressonância magnética) é uma conduta consolidada para redução da incidência de crises epilépticas em pacientes com epilepsia de difícil controle medicamentoso, também chamados farmacorresistentes. Tal procedimento encerra uma série de desafios, sendo que um deles é a determinação da zona epileptogênica, ou seja, a região cuja ressecção é necessária para resolução das crises epilépticas.

Apesar dos avanços nas tecnologias de diagnóstico por imagem, a videoeletroencefalografia (VEEG) permanece neste momento como o padrão-ouro para a seleção de pacientes e planejamento das cirurgias de epilepsia. Sua utilização em diversas situações, como no diagnóstico apurado de síndromes epilépticas que não são passíveis de cirurgia, ou mesmo de distúrbios de comportamento que não são de natureza epiléptica, a tornam extremamente valiosa para outras áreas da epilepsia que não a cirurgia.

Além disso, a imagem de ressonância magnética (IRM), que por muito tempo permaneceu como auxiliar na determinação da zona epileptogênica, tem sido cada vez mais relevante no processo de tomada de decisão cirúrgica.

Este trabalho propõe-se a estudar o quanto os resultados da videoeletroencefalografia prolongada são coincidentes com os resultados da ressonância magnética de encéfalo, quanto à determinação das zonas epileptogênicas nos pacientes com displasia cortical focal.

\subsubsection{Definições e impacto}

A epilepsia é definida, segundo a Liga Internacional contra Epilepsia (ILAE), como uma doença do cérebro definida por qualquer uma das seguintes condições: a) pelo menos duas crises epilépticas não-provocadas (ou reflexas) ocorrendo com diferença superior a 24 horas; b) uma crise epiléptica não-provocada (ou reflexa) e uma probabilidade de crises adicionais igual ao risco de recorrência geral (ao menos 60\%) depois de duas crises nãoprovocadas, ocorrendo nos próximos 10 anos; ou c) diagnóstico de uma síndrome epiléptica (FISHER et al., 2014). Por sua vez, crises epilépticas constituem alterações súbitas e temporárias do comportamento, que ocorrem devido à atividade neuronal anormalmente excessiva ou sincrônica (FISHER et al., 2005). Por sua natureza inesperada e incapacitante, elas determinam um prejuízo considerável na qualidade de vida dos pacientes (FISHER et al. 2005; FISHER et al., 2014). 
A ausência de crises por dez anos pode ser definida como epilepsia resolvida (FISHER et al., 2014), contanto que os últimos cinco anos sejam livres de medicação. Outra forma de epilepsia resolvida é para os indivíduos que possuem epilepsia autolimitada (benigna), e que tenham passado da idade de atividade da doença.

A persistência de crises epilépticas a despeito do tratamento medicamentoso adequado determina a epilepsia farmacorresistente, e implica em uma série de dificuldades, como inatividade laboral, restrição ao manejo de veículos e piora da qualidade de vida (KWAN et al., 2010), além do risco aumentado de morte precoce (CARPIO et al. 2005).

\subsubsection{Epidemiologia}

É uma enfermidade comum, afligindo cerca de 50 milhões de pessoas no mundo (SCOTT; LHATOO; SANDER, 2001). No Brasil, apresenta prevalência aproximada de $1,89 \%$ na população urbana, sendo que $45 \%$ destes são da forma ativa (BORGES et al., 2004). Para a América Latina, observou-se que a incidência observada nas Ilhas Martinica (Caribe) é de 77 casos para cada 100.000 pessoas (BURNEO; TELLEZ-ZENTENO; WIEBE, 2005 ). Corresponde a cerca de $15 \%$ das consultas no consultório do neurologista (STONE et al. 2010). Nos Estados Unidos da América, os custos anuais diretos por paciente com epilepsia são superiores a US\$11.000,00 (IVANOVA et al., 2010).

Aproximadamente $36 \%$ dos pacientes diagnosticados com epilepsia não obtêm resolução de crises epilépticas por meio do uso de um ou mais fármacos antiepilépticos (KWAN; BRODIE, 2000). Estes pacientes devem ser prontamente encaminhados a centros de diagnóstico e tratamento específicos em epilepsia (FOUNTAIN et al., 2011).

Em adultos, as causas mais comuns de epilepsia farmacorresistente são esclerose hipocampal, tumores cerebrais, displasias corticais focais e malformações corticais (VELASCO; MATHERN, 2011). Fatores de risco como parasitoses podem explicar a alta incidência de epilepsia nos países em desenvolvimento como o Brasil (LI et al. 2007). Displasia cortical, o principal substrato patológico encontrado em cirurgias de epilepsia de pacientes menores que 18 anos de idade, constitui um grupo de clara necessidade cirúrgica, uma vez que apresenta epilepsias de difícil controle em pacientes que sofrem com efeitos adversos tanto das crises quanto do seu tratamento (HAUPTMAN; MATHERN, 2012).

\subsubsection{Classificações}

A epilepsia é uma doença heterogênea, multifatorial e de prognóstico diverso. Ela compreende as mais variadas formas de manifestação clínica da crise epiléptica, que quando levadas em consideração com sua idade de ocorrência, anormalidades persistentes ao exame neurológico e características peculiares do eletroencefalograma (EEG), definem as síndromes epilépticas, ou epilepsias propriamente ditas. 
Busca-se há muitos anos uma forma adequada de se classificar tanto as crises epilépticas quanto as próprias epilepsias. A ILAE admite como válidas as classificações de 1981 para as crises COMISSION ON CLASSIFICATION AND TERMINOLOGY OF THE INTERNATIONAL LEAGUE AGAINST EPILEPSY, 1981) e de 1989 para as síndromes (COMISSION ON CLASSIFICATION AND TERMINOLOGY OF THE INTERNATIONAL LEAGUE AGAINST EPILEPSY, 1989). Discussões acaloradas têm ocorrido ao longo dos anos (ENGEL, 2006), mas ainda não há termos substitutivos validados (BERG et al., 2010).

Fundamentalmente, pode-se dividir a epilepsia em dois grandes grupos: as epilepsias focais e as epilepsias generalizadas. Da mesma forma, as crises epilépticas também podem ser divididas em crises focais (ou parciais) ou generalizadas. As epilepsias focais são aquelas cuja convergência de dados clínicos denota uma disfunção regional do cérebro. As epilepsias generalizadas, por outro lado, abrangem distúrbios difusos da atividade cerebral. Por disfunção regional, utiliza-se como parâmetro a ocorrência de crises epilépticas focais, alterações regionais ao EEG e presença de sinais focais ao exame neurológico. Acreditase que as crises focais sejam aquelas em que fenômenos de hiperssincronização cortical ocorram em determinada parte do cérebro, e envolvam de forma progressiva estruturas do mesmo hemisfério, antes de propagar por meio de conexões para o hemisfério contralateral. Por disfunção difusa, tem-se a ocorrência de crises generalizadas, alterações generalizadas ao EEG e presença de alterações difusas ao exame neurológico, ou mesmo sua normalidade. As crises generalizadas, por sua vez, envolvem rapidamente estruturas homólogas dos dois hemisférios cerebrais, e acredita-se que decorram de disfunção de canais iônicos neuronais (BERG et al., 2010).

Finalmente, determina-se se a epilepsia é secundária à lesão estrutural ou insulto cerebral prévio (sintomática); se tem substrato predominantemente genético (idiopática) ou se a causa é indeterminada (criptogênica).

\subsection{Epilepsias lesionais extrahipocampais}

\subsubsection{Importância}

O advento das imagens do parênquima cerebral nos anos 70 revolucionou o estudo da epilepsia. A neuroimagem, como é chamada, forneceu um extraordinário entendimento dos substratos patológicos da epilepsia, e isto é essencial para determinar o curso provável da doença, tratamento, possibilidade de cirurgia e prognóstico pós-operatório (VELEZRUIZ; KLEIN, 2012). O método de escolha de neuroimagem, atualmente, é a imagem de ressonância magnética de encéfalo (IRM).

Casos em que a IRM revela uma lesão estrutural apontada como causa provável da 
epilepsia focal são conhecidos como lesionais. A localização da lesão implica em diferentes apresentações clínicas. Por exemplo, a presença de uma lesão neoplásica no hipocampo pode determinar um quadro clínico de crises parciais com comprometimento da consciência sem manifestações motoras, enquanto que a mesma lesão no córtex pré-central pode gerar crises parciais motoras, sem, no entanto, existir comprometimento da consciência.

\subsubsection{Displasia cortical focal}

As displasias corticais focais (DCF) constituem um espectro de malformações do desenvolvimento cortical caracterizadas pela desorganização da arquitetura normal do córtex cerebral (CRINO, 2015). Sua associação com a epilepsia foi inicialmente descrita por Taylor (TAYLOR et al., 1971), após a demonstração de alterações focais da citoarquitetura cortical que coincidiam com o local de origem das crises epilépticas, em cirurgias de epilepsia baseadas na semiologia ictal. Sua hipótese era de que, provavelmente, se tratavam de alterações do desenvolvimento, congênitas, e que seriam responsáveis pelas crises epilépticas dos pacientes.

\subsubsection{Neuropatologia}

Várias classificações de DCF já foram propostas. A atualmente recomendada pela ILAE utiliza exclusivamente critérios histopatológicos (BLUMCKE et al., 2011). De acordo com esta classificação, existem três divisões: I, II e III.

A displasia cortical focal tipo I é caracterizada por laminação cortical anormal, e possui três subtipos: o tipo Ia é caracterizado pelo comprometimento da migração e maturação radial de neurônios; o tipo Ib pelo comprometimento da divisão em seis camadas tangenciais do córtex; e o tipo Ic pela combinação dos dois tipos anteriores. O tipo Ia possui "microcolunas" de mais de oito neurônios alinhados verticalmente. O tipo Ib não possui a composição normal de seis camadas tangenciais do isocortéx.

Alterações celulares marcam a displasia cortical tipo II. Também estão presentes alterações na laminação cortical, geralmente só sendo identificada a camada 1. Na DCF tipo IIa ocorrem neurônios citomegálicos e dismórficos. Na DCF tipo IIb, além destes neurônios anormais, são notadas as células em balão, com vários aspectos gliais. Estas células podem ser identificadas por meio de imunohistoquímica.

Quando a DCF tipo I encontra-se associada a alguma outra lesão epileptogênica, deve ser classificada como DCF tipo III, e subclassificada de acordo com a lesão associada: IIIa com esclerose hipocampal, IIIb com tumores, IIIc com malformação vascular e IIId com outras lesões adquiridas precocemente, como gliose. 


\subsubsection{Apresentação clínica}

A DCF manifesta-se com crises epilépticas em 20 a $25 \%$ dos pacientes, e destes, cerca de $76 \%$ possuem epilepsia intratável (FAUSER et al., 2006). Dos pacientes que se apresentam com epilepsia farmacorresistente, cerca de 12\% possuem DCF como etiologia à imagem de ressonância magnética (LI et al. 1995). A idade de início das crises varia de $<1$ a 60 anos de idade. Em 92,5\% dos pacientes, as crises começam antes dos 16 anos de idade. A semiologia da primeira crise depende da idade, sendo com espasmos quando iniciada antes de 1 ano de idade, passando para hipomotora até dois anos de idade, e tônica ou convulsiva daí por diante.

Não parece haver relação entre o local da DCF e a idade de início das crises, entretanto há uma relação direta entre o local da DCF e a semiologia ictal (CRINO, 2015).

Cerca de 17\% dos pacientes obtêm alívio das crises com uso de medicação (FAUSER et al., 2006). Nos demais, não há controle com medicação, sendo frequentemente indicada cirurgia de epilepsia.

Além das crises epilépticas, DCF é causa importante de comprometimento cognitivo, além de alterações comportamentais como autismo e retardo no desenvolvimento neuropsicomotor (CHEN et al. 2014).

\subsubsection{Apresentação radiológica}

Com o avanço da imagem por ressonância magnética de encéfalo na década de 1990, tornou-se aparente que a DCF era muito mais comum em pacientes com epilepsia intratável do que anteriormente imaginado (CRINO, 2015). Atualmente, a utilização da IRM é central na identificação e diagnóstico de DCF.

Preferencialmente, devem ser utilizadas sequências adequadas à epilepsia, com aquisições de imagens ponderadas $\mathrm{T} 1$ com e sem contraste paramagnético, imagens ponderadas T2 e FLAIR.

Alterações à IRM de encéfalo sugestivas de DCF incluem (MUHLEBNER et al., 2012): anormalidades nos giros; espessamento focal do córtex; apagamento da transição entre as substâncias branca e cinzenta; e alteração da intensidade do sinal cortical e subcortical. Este sinal encontra-se aumentado em sequências T2 e FLAIR, e diminuído na sequência T1 (Figura 1). Além disto, pode-se observar a presença de hipersinal subcortical a partir de um ponto do córtex (o sinal "trans-manto") em direção ao ventrículo.

Cerca de $45 \%$ dos pacientes com IRM de encéfalo sem alterações foram diagnosticados como DCF pela anatomia patológica após cirurgia de epilepsia (WANG et al., 2013), indicando que a ausência de lesões na IRM não pode afastar a hipótese de DCF como etiologia da epilepsia. 

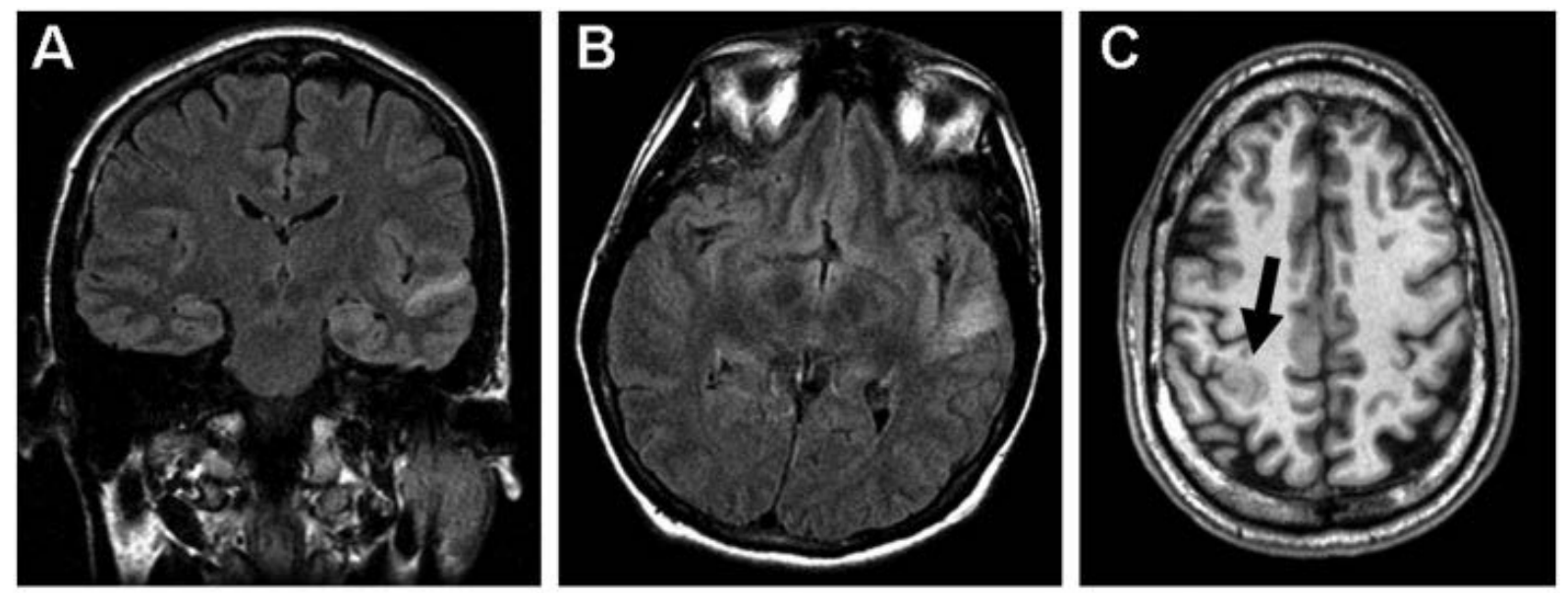

Figura 1: Imagem de ressonância magnética da displasia cortical focal, evidenciada por hipersinal na região temporal esquerda na sequência FLAIR (A,B) e hiposinal na região parietal direita na sequência T1 (C). Extraído de VELEZ-RUIZ; KLEIN, 2012 .

\subsection{Estudos em técnicas diagnósticas}

Similar à pesquisa terapêutica, os estudos em técnicas diagnósticas devem seguir alguns passos (SCHAAFSMA et al., 2009). O primeiro passo é a avaliação das características intrínsecas de um novo teste, como sua sensibilidade e especificidade, que definem o alcance clínico da tecnologia. O próximo passo é a estimativa do valor agregado ao diagnóstico, uma vez que testes diagnósticos são normalmente usados sequencialmente e dessa forma, a informação adicional oferecida ao tomador de decisão deve ser considerada; o terceiro passo é a análise do efeito no desfecho clínico, pois a justificativa para novos testes diagnósticos geralmente é o acesso potencial a tratamentos benéficos; e o último passo compreende o cálculo da relação custo-benefício, que é a forma de agregar toda a informação coletada previamente.

Convergência é a situação em um teste apresenta determinado resultado, que é o mesmo encontrado em outro teste. Entretanto, esta convergência nem sempre indica que os testes estão corretos, pois ambos podem concordar com um resultado errado. A acurácia, por sua vez, é a propriedade que um teste possui de concordar com a condição clínica "verdadeira". Comparando-se testes quanto à acurácia, pode-se determinar se um é equivalente ou não em termos diagnósticos a outro.

No presente trabalho, os resultados avaliados são as zonas epileptogênicas presumidas, regiões que devem ser ressecadas para que o paciente com epilepsia alcance uma vida livre de crises epilépticas.

Embora aparentemente muito direta, uma variação considerável nos desenhos e descrições dos estudos de técnicas diagnósticas é observada. Para aumentar a consistência da metodologia foi lançada a iniciativa STARD (Standards for Reporting of Diagnostic 
Accuracy Studies) (BOSSUYT et al., 2015).

Para a cirurgia de epilepsia, a "condição clínica verdadeira", ou padrão-ouro para a determinação da zona epileptogênica é o resultado da discussão das avaliações préoperatórias em reunião multidisciplinar (BENICZKY et al., 2013), complementado pela informação de desfecho pós-operatório após 12 meses de cirurgia (BURCH et al., 2012).

Um modelo de decisão analítico é uma das ferramentas que podem ser utilizadas para guiar a aplicação de um teste diagnóstico. Por meio deste modelo, desfechos clínicos após a aplicação de um novo teste podem ser preditos pela interpretação da literatura disponível. Sem dúvida, um estudo randomizado controlado é a melhor forma de comparação entre testes, entretanto esta é uma possibilidade muitas vezes impraticável, devido à grande quantidade de sujeitos envolvidos e ao longo período de acompanhamento, que podem fazer com que o teste em estudo esteja obsoleto ao final (SCHAAFSMA et al., 2009).

\subsubsection{Ressonância magnética de encéfalo}

\subsubsection{Desenvolvimento}

O prêmio Nobel em Medicina e Fisiologia de 2003 foi concedido aos Drs. Peter Mansfield e Paul Lauterbur por seu trabalho utilizando ressonância nuclear magnética (RNM) para a geração de imagens de tecidos humanos (BOESCH, 2004).

Isidor Rabi é creditado como o descobridor da RNM, nos anos 30. Na tentativa de medir as propriedades magnéticas de vários elementos, incluindo hidrogênio, deutério e lítio, seu grupo descreveu como núcleos podem ser induzidos a mudar sua orientação magnética principal por um campo magnético oscilante. Na década de 40, Bloch e Purcell aplicaram a RNM em matéria condensada (como parafina). Até então, a RNM era largamente um conceito teórico, limitado a experimentos de física nuclear (RAMSEY, 1999). Por seus avanços, foram agraciados com o prêmio Nobel de Física em 1952 (BOESCH, 2004).

Por muito tempo, a RNM foi utilizada principalmente para que fossem determinados os componentes de soluções diluídas em tubos de ensaio, para a realização de estudos químicos. Nos anos 70, Lauterbur mostrou que aplicando RNM em tecidos ricos em água poderiam ser geradas imagens, baseadas na forma com que os átomos de hidrogênio reagissem a um campo magnético. O segredo para que isso acontecesse eram gradientes, pequenas variações no campo magnético que distinguiam átomos de hidrogênio em diferentes partes de uma amostra. Aplicando estes gradientes de campos magnéticos em diferentes direções através de uma amostra e combinando os sinais de RNM resultantes, foi possível a construção de imagens que determinavam a localização tridimensional dos núcleos de hidrogênio, gerando as imagens de ressonância magnética (IRM) (LAUTERBUR, 1973; BOESCH, 2004).

A melhoria na aquisição de sinais de RNM, com elaboração de imagens em tempo 
hábil e com superior resolução, foi a contribuição de Mansfield ao desenvolvimento da IRM (MANSFIELD; MAUDSLEY, 1977), e o que a levou a ser adotada amplamente pela medicina clínica, especialmente pela neurologia.

\subsubsection{Fundamentos}

O princípio fundamental da IRM é que, se certos núcleos são colocados em um campo magnético, eles são capazes de "absorver" energia em uma determinada radiofrequência, e sinais podem ser adquiridos como resultado dessa troca de energia à medida que os núcleos retornam ao seu estado original (BLOCH, 1946).

Um grande número de técnicas podem ser utilizadas para gerar uma IRM e para o aprimoramento do contraste, que permitirão que áreas doentes do cérebro possam ser identificadas. O exame por IRM depende, portanto, da informação sobre o que é mais importante para se identificar.

Para o máximo de qualidade na imagem, é desejado que exista uma boa relação sinal-ruído, alta resolução, bom contraste entre as diferentes estruturas que se deseja visualizar, ausência de artefatos e um tempo curto para aquisição de imagens. Campos magnéticos de alta potência, com 1,5 a 3T, são usualmente utilizados na atualidade.

Os tempos de relaxação T1 e T2 são o tempo necessário para o retorno dos spins dos átomos de hidrogênio ao seu estado de equilíbrio, após um pulso de radiofrequência. O T1 se refere ao tempo de relaxação longitudinal, e diferenças no T1 de ressonância da água em vários tecidos formam a base de muitas sequências de imagens para descriminar o liquido cefalorraquidiano (T1 longo) da substância cinzenta (T1 curto) e substância branca (T1 mais curto).

O tempo T2 refere-se ao tempo de relaxação transversal, e é independente da relaxação T1. É fundamental para a interpretação de imagens, pois gera grande parte do contraste necessário para comparação com imagens T1 e determinação de áreas patológicas (JACKSON; KUZNIECKY; PELL, 2005).

Sequências de pulso são desenvolvidas continuamente, manipulando T1 e T2 com finalidades bem específicas. Entre elas, destaca-se o FLAIR (fluid-attenuated inversion recovery), uma sequência de inversão-recuperação em que imagens possuem um T1 bem longo, de forma a atenuar o sinal de líquidos na imagem. Seu objetivo é de demonstrar lesões corticais e periventriculares que poderiam ser mascaradas na sequência T2 (JACKSON; KUZNIECKY; PELL, 2005).

\subsubsection{Indicações}

A IRM é considerada, atualmente, uma tecnologia-chave no manejo de pacientes com epilepsia, principalmente naqueles candidatos à cirurgia (WELLMER et al., 2013). 
Entretanto, peculiaridades desta doença fazem com que seja necessário o estabelecimento de protocolos específicos, que visam a aumentar a quantidade de lesões detectadas, e melhor identificação de estruturas críticas saudáveis. Além disso, o tempo e custo associados ao procedimento devem ser observados, pois trata-se de doença comum. Máquinas com potência inferior a 1,5T são consideradas obsoletas para investigação de epilepsia (WELLMER et al., 2013).

A sequência FLAIR é mais importante para identificação de lesões epileptogênicas, identificando 84,8\% delas, seguida por T2 (62,51\%), T1 (2,98\%), IR (inversion-recovery; $2,98 \%$ ) e difusão $(0,36 \%)$ (WELLMER et al., 2013).

Algumas lesões epileptogênicas com implicações terapêuticas, como cavernomas e displasias corticais, possuem entre 5 e $7 \mathrm{~mm}$ de diâmetro, portanto a espessura de corte deve ter valor igual a $3 \mathrm{~mm}$, sem intervalos. A angulação do corte para imagens T1 utiliza a linha intercomissural (entre comissura anterior e posterior). Imagens T2 e FLAIR devem ser anguladas perpendiculares ao eixo hipocampal, seja no plano coronal ou axial. Em especial, a sequência T1 deve ser registrada em 3D, com espessura de 1 $\mathrm{mm}$, sem intervalos. Finalmente, deve-se acrescentar sequência adequada à visualização de calcificações e hemossiderina, como gradiente-echo, apenas no plano axial e perpendicular ao eixo hipocampal (WELLMER et al., 2013).

Trata-se de exame custeado pelo sistema de saúde público brasileiro (SUS), sendo pago valor de $\mathrm{R} \$ 268,75$ por cada exame de encéfalo (SIGTAP, 2015).

\subsubsection{Videoeletroencefalografia prolongada}

\subsubsection{Desenvolvimento}

A associação do registro eletroencefalográfico (EEG), desenvolvido por Hans Berger em 1929 (BERGER, 1935), com o registro em vídeo das crises epilépticas foi descrita por Ives (IVES; THOMPSON; GLOOR, 1976), relatando a experiência do Montreal Neurological Institute na observação de pacientes com epilepsia sendo considerados para cirurgia desde o início dos anos 70. Este avanço se deveu em grande parte à evolução do registro digital do eletroencefalograma, e parte da técnica utilizada havia sido descrita uma década antes, quando a telemetria para EEG despertava interesse nos militares envolvidos na chamada "corrida espacial" (KAMP, 1963). Em Montreal, eram necessárias três salas para acomodação de paciente, computador e eletroencefalógrafo. O tempo máximo de registro eletroencefalográfico digital de uma crise epiléptica era de 4 minutos, e a interpretação do traçado ainda não era em tela, mas sim registrada em papel. Os registros em vídeo eram analisados separadamente, tendo como referência marcos de tempo que eram idênticos aos registrados no EEG (IVES; THOMPSON; GLOOR, 1976). 


\subsubsection{Fundamentos}

A videomonitorização eletroencefalográfica (VEEG) consiste no registro simultâneo e sincronizado do EEG e do comportamento clínico do paciente por períodos prolongados, para avaliação de distúrbios paroxísticos da função cerebral. Seu tempo de avaliação é variado, mas frequentemente mais longo que o EEG de rotina ambulatorial, eventualmente alcançando semanas e necessitando, portanto, de internação hospitalar. Atualmente, modelos digitais compactos e integrados de EEG e vídeo são o padrão da indústria, sem necessidade da impressão em papel do EEG ou análise de vídeo em mídia distinta (AMERICAN ELECTROENCEPHALOGRAPHIC SOCIETY, 2008).

Durante a internação, eventualmente a medicação antiepiléptica é reduzida, para favorecer a ocorrência de eventos paroxísticos, epilépticos ou não, que levaram à internação do paciente. O problema desta conduta é que expõe o paciente à possibilidade de crises epilépticas mais graves que as habituais, além de uma série de intercorrências psiquiátricas, como psicose ou ansiedade. A chance de ocorrência de eventos adversos é considerada baixa $(<10 \%)$ e geralmente os eventos são leves (LIU; MENG; LIU, 2012; RIZVI et al., 2014: LEY et al., 2014).

Para a cirurgia de epilepsia, a VEEG é, no momento, instrumento praticamente indispensável para tomada de decisão (ROSENOW; LUDERS, 2001). Como a decisão cirúrgica depende da confirmação que a doença do paciente é epilepsia, e que o local de origem das crises coincide com a área a ser operada, torna-se necessário o registro das crises epilépticas para determinar a conduta cirúrgica.

\subsubsection{Indicações}

São duas as principais indicações para a realização da VEEG: a primeira é a caracterização que anormalidades de comportamento e/ou eletroencefalográficas paroxísticas são de natureza epiléptica. Isto inclui o registro de crises epilépticas com documentação de descargas epileptiformes interictais e ictais. Estes dados auxiliam na diferenciação entre epilepsias e condições não-epilépticas, como síncope, arritmias cardíacas, narcolepsia, crises psicogênicas), e são conhecidos como estudos diagnósticos (AMERICAN ELECTROENCEPHALOGRAPHIC SOCIETY, 2008). A segunda é a localização, em paciente sabidamente com epilepsia, do local de origem das crises, e sua utilização em plano de cirurgia de epilepsia, conhecidos como estudos pré-cirúrgicos.

Como é necessária a supervisão do paciente e de seu registro por longos períodos, utilizando equipamentos avançados e sensíveis, a VEEG é considerada um exame de alto custo. O sistema único de saúde brasileiro (SUS) paga atualmente $\mathrm{R} \$ 1.707,05$ pelo exame com duração de $72 \mathrm{~h}$ (SIGTAP, 2015), cerca de $\mathrm{R} \$ 569,00$ por dia, por leito. 


\subsection{Cirurgia de epilepsia lesional extrahipocampal}

\subsubsection{Desenvolvimento}

Desde o período neolítico (3000 a.C.), o homem tenta resolver a epilepsia com intervenções cirúrgicas, ainda que nessa época a doença fosse considerada uma entidade mágica, sobrenatural. Essa visão permaneceu até os anos 400 a.C., quando Hipócrates descreveu a epilepsia como uma doença, e que tinha origem no cérebro (SCHIJNS et al., 2015). Nessa época, trepanações eram utilizadas como cirurgias de epilepsia.

Na segunda metade do século XIX, John Hughlings Jackson descreveu crises epilépticas com presença de fenômenos psíquicos e experienciais que associou a lesões no lobo temporal (TAYLOR; MARSH, 1980), iniciando a correlação da fenomenologia ictal com disfunção cerebral. Por sua vez, Victor Horsley, em 1886, localizou e retirou lesões epileptogênicas em nove pacientes com crises parciais (HORSLEY, 1887), promovendo o início das cirurgias cerebrais de epilepsia.

A difusão da eletrocorticografia (ECoG), o registro intraoperatório da atividade elétrica cerebral com utilização de eletrodos repousados sobre o córtex cerebral por Penfield e Jasper nos anos 40 (ANDERMANN, 2000), foi determinante para o sucesso das primeiras cirurgias de epilepsia, que não contavam com recursos de imagem capazes de determinar com precisão o local da lesão cerebral, devendo portanto a escolha do local a ser operado baseada na análise visual do córtex e da atividade epileptiforme à ECoG.

\subsubsection{Fundamentos}

Durante a investigação de epilepsias farmacorresistentes visando cirurgia, é fundamental que o paciente seja abordado com exames adequados (ROSENOW; LUDERS, 2001: SCHUELE; LUDERS, 2008). O exame neurofisiológico utilizado é a videoeletroencefalografia (VEEG), que consiste nos registros audiovisuais e por eletroencefalograma, simultâneos e sincronizados, das crises epilépticas do paciente. Para isto é necessária sua internação hospitalar, pois o registro em tempo hábil depende da retirada gradual dos fármacos antiepilépticos, e, portanto, o risco de estado de mal epiléptico é aumentado (DOBESBERGER et al., 2011).

Ao final da investigação, deve-se agrupar os achados em seis grandes grupos, cuja resultante determina o que pode ser feito em termos cirúrgicos: a zona irritativa, a zona de início ictal, a zona de déficit funcional, a zona sintomatogênica, a lesão epileptogênica e a zona epileptogênica (ROSENOW; LUDERS, 2001).

A zona irritativa compreende a região do cérebro onde ocorrem os paroxismos epileptiformes interictais (WESTMORELAND, 1998). Trata-se de indício de onde, supostamente, o cérebro teria maior propensão a originar crises epilépticas. Entretanto, muitas 
vezes isto não é verdadeiro (CATARINO; VOLLMAR; NOACHTAR, 2012).

A zona de início ictal é o local onde se presume que ocorra o início da crise epiléptica. Para sua determinação, utiliza-se inicialmente o EEG de escalpe, apesar das limitações existentes em sua resolução espacial (FOLDVARY et al., 2001). Compara-se a atividade eletrográfica ictal com manifestações clínicas ictais do paciente, e determina-se se há convergência. Assim, pode-se inferir em qual região do cérebro está começando a crise epiléptica. Algumas vezes, isto não se mostra possível, e é necessário registro invasivo por meio de eletrodos intracranianos para explorar a zona de início ictal com mais acurácia (KUTSY; FARRELL; OJEMANN, 1994; TAUSSIG; MONTAVONT; ISNARD, 2015).

A zona de déficit funcional é aquela em que há provas de que os neurônios de determinada parte do cérebro estão comprometidos, portanto poderiam estar envolvidos na epilepsia. Determina-se esta região por intermédio do exame neurológico, buscando sinais focais, ou exame neuropsicológico, onde se testam domínios cognitivos (HELMSTAEDTER, 2004). Além destes, exames de neuroimagem funcional como PET e SPECT também são capazes de determinar hipometabolismo interictal ou hiperperfusão ictal focal e ajudam na abordagem cirúrgica da epilepsia (KUMAR; CHUGANI, 2016).

A zona sintomatogênica é aquela responsável pela manifestação clínica da crise epiléptica do paciente, e é avaliada por meio do registro ictal em vídeo. Assume-se atualmente que redes neurais estão permanentemente ativadas na propagação de crises epilépticas (BERG et al., 2010); desta forma algumas vezes a semiologia ictal pode falsamente regionalizar uma zona de início ictal (MANFORD; FISH; SHORVON, 1996; $\mathrm{SO}, 2006$ ). Por outro lado, vários sinais clínicos validados permitem determinar em qual hemisfério cerebral houve início ictal (sinais lateralizatórios), que são valiosos para a abordagem cirúrgica da epilepsia (LODDENKEMPER; KOTAGAL, 2005).

Lesões epileptogênicas são aquelas, identificadas à ressonância magnética (RM), que são comumente associadas à epilepsia. Além de boa acurácia etiológica, técnicas modernas de RM permitem determinar também qual a relação da lesão com estruturas eloquentes, como área de Broca, Wernicke e giro pré-central (URBACH, 2005).

Finalmente, zona epileptogênica compreende a região que deve ser ressecada à cirurgia para que ocorra cura das crises epilépticas (ROSENOW; LUDERS, 2001). Sua localização provável deve ser determinada antes da cirurgia, porém pode ser ajustada por meio de exames adicionais que visam ao refinamento do procedimento, como eletrocorticografia (permite determinar zona irritativa diretamente no córtex durante cirurgia) e estimulação cortical (determina área eloquentes). 


\subsubsection{Indicações}

O paciente é candidato à cirurgia de epilepsia quando tem seu diagnóstico confirmado e sua zona epileptogênica presumida com uso da videoeletroencefalografia. Além disso, deve apresentar crises epilépticas a despeito do uso correto de duas medicações antiepilépticas adequadas. Deve concordar com a proposta cirúrgica, quando informado dos riscos inerentes ao procedimento de grande porte, e do possível comprometimento neuropsicológico e neurológico associado (DASH et al., 2012).

Após procedimento, mantém acompanhamento especializado em serviços com experiência em cirurgia de epilepsia.

\subsection{Perguntas do trabalho atual}

No manejo de pacientes com epilepsia farmacorresistente lesional sendo considerados para cirurgia, frequentemente observa-se que os resultados da VEEG e da IRM são convergentes, ou seja, o resultado de um é bastante parecido com o resultado do outro, quanto à localização da zona epileptogênica. Entretanto, estudos para determinar com que frequência esta situação ocorre são escassos (BURCH et al., 2012).

Desta forma, pergunta-se: com que frequência há convergência dos resultados da videoeletroencefalografia prolongada e da ressonância magnética de encéfalo em pacientes com epilepsia farmacorresistente associados à displasia cortical focal sendo considerados para cirurgia? Qual o desempenho diagnóstico de cada um destes exames para a determinação da zona epileptogênica? 



\section{Objetivos}

\subsection{Objetivo principal}

Determinar, nos pacientes com epilepsia lesional extrahipocampal farmacorresistente com displasia cortical focal à IRM, submetidos à avaliação pré-cirúrgica, se os resultados da videoeletroencefalografia prolongada e da imagem de ressonância magnética de encéfalo possuem convergência de resultados, e com que frequência isto ocorre.

\subsection{Objetivo secundário}

Determinar e comparar o desempenho diagnóstico da videoeletroencefalografia prolongada e da ressonância magnética de encéfalo, verificando a acurácia quanto à determinação da zona epileptogênica, em pacientes com epilepsia lesional extrahipocampal farmacorresistente com displasia cortical focal à IRM, submetidos à avaliação pré-cirúrgica. 

Parte II

Desenvolvimento 



\section{Participantes e métodos}

\subsection{Desenho do estudo}

Um estudo observacional retrospectivo clínico de acurácia foi realizado, com análise dos laudos videoeletroencefalográficos e de ressonância magnética de encéfalo por meio de revisão de prontuário, relacionando os achados com o local em que ocorreu a cirurgia, informação que é resultante da reunião clínica multidisciplinar pré-operatória do CIREP (Centro de Cirurgia de Epilepsia) e que foi obtida das descrições cirúrgicas de cada caso. A coleta dos dados ocorreu nas dependências do Hospital das Clínicas de Ribeirão Preto, no período de fevereiro de 2013 a julho de 2014. A revisão de prontuários foi conduzida nas salas do SAME (Serviço de Arquivo Médico) do HC USP Ribeirão. A redação deste estudo procurou adequar-se à iniciativa STARD (Standards for Reporting of Diagnostic Accuracy Studies) para divulgação científica (BOSSUYT et al., 2015). Determinou-se que o padrão-ouro seria o local da cirurgia nos pacientes operados com desfecho conhecido após 12 meses de cirurgia (BURCH et al. 2012), e os testes-índice seriam a IRM e a VEEG.

\subsubsection{Critérios de inclusão}

Foram incluídos pacientes com as seguintes características:

- Laudo de videoeletroencefalografia prolongada indicando zona de início ictal presente no prontuário;

- Laudo de ressonância magnética do encéfalo presente no prontuário;

- Referência em laudo radiológico de lesão epileptogênica extrahipocampal à imagem de ressonância magnética de encéfalo, compatível com displasia cortical focal;

- Se operado, submetido à cirurgia de epilepsia no HC USP Ribeirão.

\subsubsection{Critérios de exclusão}

Foram excluídos pacientes com qualquer uma das seguintes características:

- Mais que uma lesão epileptogênica à imagem de ressonância magnética de encéfalo;

- Ausência do laudo de videoeletroencefalografia no prontuário;

- Ausência de lesão epileptogênica à imagem de ressonância magnética de encéfalo; 
- Ausência de registro de crises epilépticas durante videoeletroencefalografia prolongada;

- Cirurgia de epilepsia prévia;

- Avaliação durante estado de mal epiléptico.

\subsection{Identificação da amostra}

O primeiro passo foi a identificação dos pacientes que teriam seus prontuários avaliados. Para isto, foi consultado o banco de dados do serviço de Cirurgia de Epilepsia (CIREP) do Hospital das Clínicas de Ribeirão Preto (HC USP Ribeirão), cobrindo o período de 1997 a 01/03/2012 e visando-se à busca de pacientes que foram investigados pela VEEG na unidade, e que correspondessem aos critérios de inclusão no estudo. Se fosse incluído, o paciente teria seu registro anotado para posterior revisão de prontuário. Os prontuários foram avaliados em ordem alfabética, e pertenciam a uma série consecutiva de pacientes que atendiam aos critérios de inclusão.

\subsection{Revisão do prontuário médico}

A revisão de prontuários ocorreu no Serviço de Arquivo Médico (SAME) do HC USP Ribeirão. A partir de modelo estruturado para entrada de dados (Figura 2), foram feitas anotações acerca das condições demográficas e clínicas do paciente, e seu desfecho pósoperatório segundo a escala de Engel (WIESER et al., 2001). A busca pelos dados clínicos foi feita estritamente pela leitura dos laudos de VEEG e IRM presentes no prontuário.

Foram anotados dados demográficos, como registro no HC USP Ribeirão, gênero, data de nascimento, data da primeira consulta, idade de início da epilepsia, data de finalização do VEEG, data da última consulta ambulatorial, se houve recorrência de crises e sua data. Finalmente, o desfecho foi classificado de acordo com a escala de Engel (WIESER et al., 2001), aplicada nos retornos ambulatoriais após a cirurgia. Esta escala compreende as seguintes classes:

1. Engel I (livre de crises incapacitantes):

a) completamente livre de crises desde cirurgia;

b) crises parciais simples não incapacitantes;

c) algumas crises incapacitantes após a cirurgia, mas livre delas há pelo menos 2 anos;

d) convulsões apenas com retirada de fármaco anti-epiléptico. 
2. Engel II (raras crises incapacitantes):

a) raras crise incapacitantes desde cirurgia;

b) mais do que raras crises incapacitantes inicialmente, mas agora crises raras há pelo menos 2 anos;

c) apenas crises noturnas.

3. Engel III (melhora significativa):

a) redução importante na freqüência de crises;

b) intervalos livres de crises prolongados, somando mais que metade do período de acompanhamento há pelo menos 2 anos.

4. Engel IV (sem melhora significativa).

Para determinação do desfecho, foram anotados os dados presentes no prontuário do paciente. Se houvesse discordância, o dado mais recente era anotado. Neste estudo, não foram consideradas as subclasses da escala de Engel, apenas as classes livre de crises (Engel I) ou não-livre de crises (agrupando as classes Engel II, III e IV).

Além disso, foram anotados os dados do laudo do EEG interictal, se presente, fazendo referência à localização e hemisfério cerebral de descargas epileptiformes. Se houvesse mais que uma localização diferente em dois exames, o resultado foi considerado como difuso. Se houvesse mais que um hemisfério em dois exames, o resultado foi considerado como não-lateralizatório.

Todos os laudos foram assinados por médicos supervisores de seus respectivos serviços. A aquisição de sinais eletroencefalográficos utilizou o padrão do serviço, com utilização de montagem 10-20 com inclusão de eletrodos intermediários temporais (KLEM et al., 1999). A ressonância de encéfalo obedeceu ao protocolo de epilepsia, estabelecido pelo serviço de radiologia local. Os médicos que assinaram o laudo de IRM desconheciam a condição clínica do paciente. Por outro lado, os médicos que assinaram o laudo de VEEG poderiam ter verificado o laudo de IRM, que geralmente é realizada antes da internação.

A data da cirurgia de epilepsia, se ocorrida, também foi anotada, assim como a localização e hemisfério de retirada do tecido cerebral, baseada nos relatórios de cirurgia. Se o paciente fosse submetido à monitorização invasiva, seja ela eletrocorticografia aguda ou monitorização crônica, era feita anotação sobre posicionamento dos eletrodos e se havia sido realizada estimulação cortical.

A região e hemisfério cerebral da lesão epileptiforme à ressonância magnética foi anotada, baseada exclusivamente no laudo presente no prontuário, assim como a região e hemisfério cerebral da zona de início ictal observada à videoeletroencefalografia prolongada. 

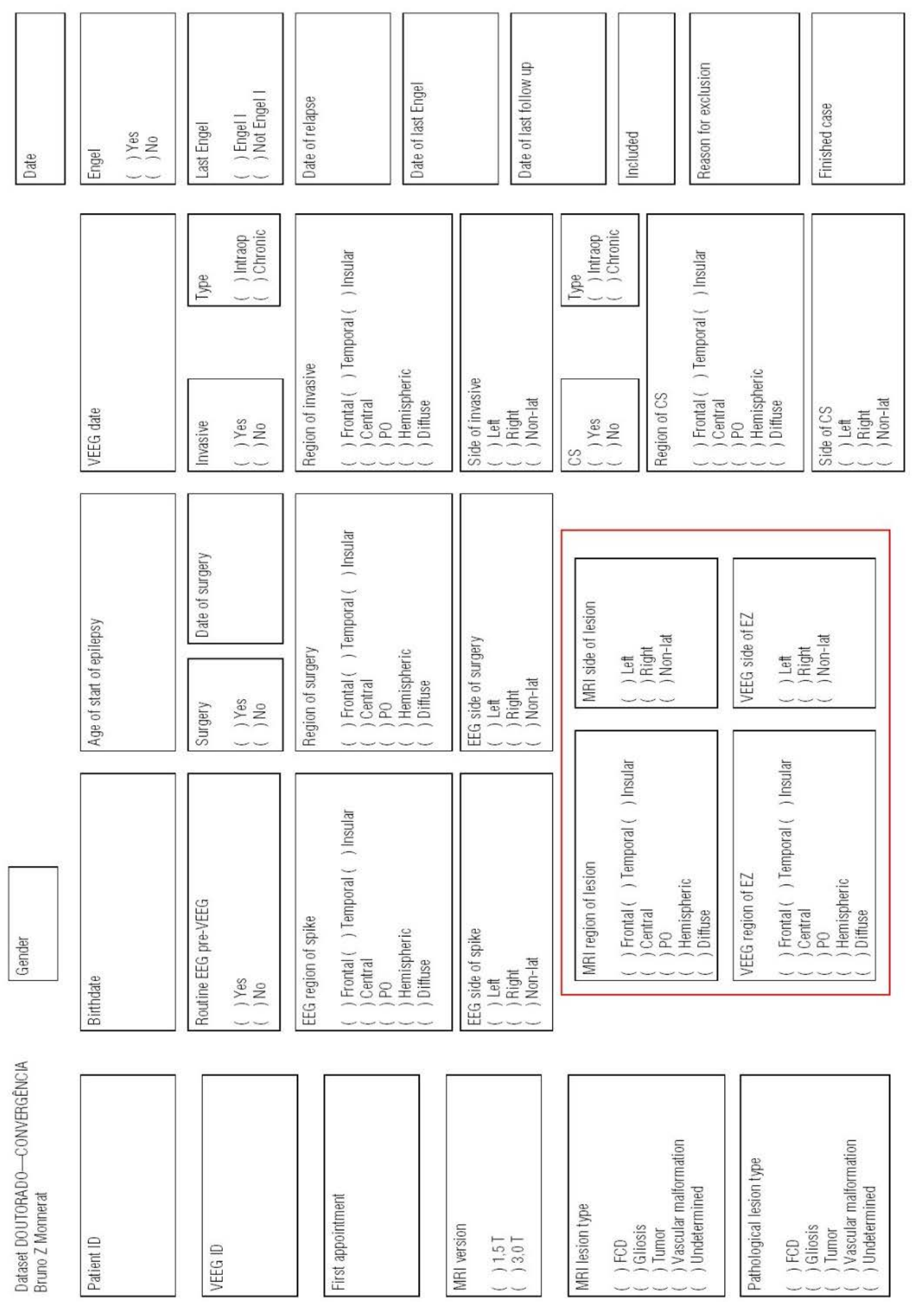

Figura 2: Instrumento para captação de dados do prontuário médico. 


\subsection{Análise estatística}

A análise estatística foi realizada com o programa SPSS Statistics 22 (IBM Corp., Armonk/USA) para Windows e uma calculadora clínica disponível na internet (VASSARSTATS, 2015). Foram considerados estatisticamente significantes os resultados cujo valor-p foram menores que 0,05. Utilizou-se o teste de McNemar para análise de dados medidos em uma escala nominal e apresentados em tabelas cruzadas. Para variáveis contínuas, foi aplicado o teste t para amostras pareadas.

Para determinação da acurácia diagnóstica da zona de início ictal à VEEG e da localização da lesão epileptogênica à IRM, tendo em perspectiva o local em que foi realizada a cirurgia e o desfecho pós-operatório, cada exame foi avaliado inicialmente de forma independente, orientando os resultados em uma tabela cruzada.

A sensibilidade foi calculada como a porcentagem de pacientes verdadeiro-positivos (ou seja, pacientes cuja localização da zona de início ictal ou da lesão epileptogênica concordava com o local onde foi realizada cirurgia, e que estavam livres de crises), dividido pelo total de pacientes livres de crises epilépticas (com ou sem concordância). A especificidade foi calculada de acordo com a fórmula: número de verdadeiro-negativos/(número de verdadeiro-negativos + número de falso-positivos). Neste caso, verdadeiro-negativos foram considerados quando havia discordância da zona de início ictal ou da lesão epileptogênica com o local de realização da cirurgia, e os pacientes se encontravam sem vida livre de crises. Falso-positivos foram caracterizados como os pacientes em que a cirurgia foi conduzida no local de convergência, porém não há vida livre de crises. Falso-negativos são os casos em que a cirurgia foi conduzida em local de divergência, mas houve vida livre de crises (BURCH et al., 2012).

Para avaliação da concordância entre os exames de VEEG e IRM em comparação com o índice de referência (local da cirurgia) foi utilizada a estatística kappa de Cohen (LANDIS; KOCH, 1977). Os valores de kappa foram interpretados quanto à concordância em agrupamentos convencionais: pobre $(\mathrm{k}<0)$; leve $(0,01-0,2)$; razoável $(0,21-0,4)$; moderada $(0,41-0,6)$, substancial $(0,61-0,8)$ e quase perfeita $(>0,8)$.

Valores preditivos foram calculados baseados no desfecho pós-operatório. Valores preditivos positivos (VPP) foram calculados como a porcentagem de pacientes com desfecho pós-operatório livre de crises/porcentagem de pacientes não-livres de crises quando havia concordância do exame em análise com o local da cirurgia. O valor preditivo negativo (VPN) foi calculado como a porcentagem de pacientes que não estavam livres de crise quando não havia concordância do exame em análise com o local da cirurgia (BENICZKY et al., 2013).

Finalmente, para estimar o valor clínico de cada um dos exames em questão (VEEG e IRM), foram calculadas razões de verossimilhança baseados em tabelas cruzadas $(\overline{\mathrm{BE}}-$ 
NICZKY et al. 2013). A tabela foi completada com dados de concordância e discordância de cada um dos exames, tendo em perspectiva a ocorrência ou não de vida livre de crises. Desta forma, para os casos concordantes, as razões de verossimilhança foram: (número de concordantes livres de crise/total livre de crise)/(número de concordantes não-livres de crises/total não-livre de crises). Para os casos discordantes: (número de discordantes livres de crises/total livres de crises)/(número de discordantes não-livres de crises/total não-livres de crises).

Quando um teste apresenta uma alta especificidade e uma alta sensibilidade em comparação a outro, este teste é claramente superior. Porém, quando um teste possui maior sensibilidade e o outro teste maior especificidade, as conclusões não são tão claras, e métodos especiais de comparação devem ser utilizados. Dois índices sumários de comparação foram utilizados no trabalho: o índice de Youden (YOUDEN, 1950) e a área sob a curva ROC (BIGGERSTAFF, 2000). O uso do índice de Youden é uma novidade nos trabalhos em cirurgia de epilepsia. Este índice representa a diferença entre a taxa de verdadeiros positivos e a taxa de falsos negativos, apresentando resultados que variam entre zero e 1 . Quanto maior o valor, melhor o desempenho diagnóstico do teste. A área sob a curva ROC é o instrumento convencional na avaliação de testes, e sua relação com o índice de Youden é que este último pode determinar o ponto de corte ideal na curva ROC em estudos de dados contínuos (TONG et al., 2016). O uso de vários índices no presente estudo teve como objetivo o aumento da robustez das conclusões.

A escolha do local onde foi realizada cirurgia como método de referência procurou resolver imperfeições associadas a outro método de referência, que seria a decisão da reunião clínica multidisciplinar. Escolhendo a cirurgia como referência, pode-se utilizar o acompanhamento clínico pós-operatório como suplemento, melhorando a acurácia do método de referência ao qual o teste-índice está sendo comparado (BURCH et al., 2012).

\subsection{Aprovação do comitê de ética em pesquisa}

O presente trabalho foi aprovado pelo CEP (Comitê de Ética em Pesquisa) do HC USP Ribeirão por intermédio do parecer $n^{\circ} 1.335 .345$ de 24 de novembro de 2015, com registro na Plataforma Brasil/CAAE 45858415.0.0000.5440. Foi dispensada a aplicação de termo de consentimento livre e esclarecido (Anexo A).

\subsection{Declaração de conflitos de interesse}

O autor declara ter sido neurologista assistente do Centro de Cirurgia de Epilepsia do Hospital das Clínicas de Ribeirão Preto até dezembro de 2013, e seu orientador é o diretor do referido Centro. Atualmente, o autor não possui vínculo empregatício com a 
instituição.

\subsection{Custeio}

Não houve qualquer tipo de suporte financeiro específico para o desenvolvimento deste trabalho. 



\section{Resultados}

\subsection{Participantes do estudo}

A análise do banco de dados do CIREP revelou 2126 exames de VEEG até 01/03/2012. Desses, 845 possuíam descrição de IRM com ausência de lesão hipocampal. Oitenta e um exames eram repetidos, portanto 764 exames pertenciam a pacientes com possível lesão extrahipocampal à IRM. Foram revisados 209 prontuários $(27,35 \%$ do desejado), devido à restrição de tempo.

Dos 209 pacientes, 97 foram excluídos. Destes, 64 foram excluídos por apresentarem laudo de IRM indicando lesão temporal mesial $(\mathrm{n}=26)$, lesão subcortical $(\mathrm{n}=2)$, dupla lesão epileptogênica $(n=10)$, malformação cortical difusa $(n=9)$ ou resultado normal $(n=17)$. Adicionalmente, havia 13 pacientes sem laudo de VEEG no prontuário. Sete pacientes não apresentaram crises epilépticas durante VEEG. Um paciente foi avaliado durante estado de mal epiléptico. Em 12 pacientes, o VEEG foi realizado após cirurgia de epilepsia.

Tiveram a elegibilidade confirmada, portanto, 112 pacientes. Destes, não foram avaliados 54 pacientes com etiologias diferentes de DCF, também conforme laudo de IRM: gliose $(n=25)$, tumor $(n=17)$, malformação vascular $(n=6)$, outras lesões $(n=4)$ e lesão indeterminada $(\mathrm{n}=2)$.

Foram, portanto, avaliados neste estudo 58 pacientes com DCF identificada à IRM, que possuíam laudo da VEEG indicando a zona de início ictal. Este grupo foi considerado para a análise descritiva e para o teste de convergência. Em seguida, 43 pacientes que possuíam em comum o fato de terem sido submetidos à cirurgia de epilepsia e apresentarem acompanhamento pós-operatório superior a 12 meses (Figura 3) foram adicionalmente avaliados para os cálculos de acurácia diagnóstica da VEEG e da IRM.

\subsection{Dados descritivos}

Foram então analisados os prontuários de 58 pacientes com DCF identificada à IRM e submetidos à investigação pré-cirúrgica no CIREP. Dois pacientes não foram submetidos à cirurgia de epilepsia.

A idade mediana à VEEG foi de 16 anos, tendo o paciente mais jovem 1 ano de idade e o mais velho 58 anos. A maioria dos pacientes submetidos à VEEG tinha menos de 26 anos. A idade mediana à cirurgia foi de 15,5 anos. O paciente mais jovem possuía 2 anos de idade, e o mais velho 58 anos. A maioria (75\%) dos pacientes operados tinha menos que 28 anos (Figura 4). Havia 30 mulheres (51,7\%) na amostra. 
2126 exames de VEEG no CIREP anteriores a 01/03/2012.

1281 exames com lesão hipocampal na IRM

845 exames de VEEG sem lesão hipocampal à IRM

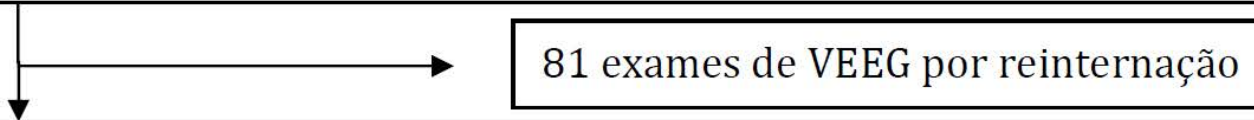

764 exames de VEEG com possível lesão extrahipocampal à IRM

209 prontuários revisados (27,35\% do desejado)

Excluídos (n=97)

Lesão subcortical $(\mathrm{n}=2)$

Malformação cortical difusa $(n=9)$

Sem crise epiléptica $(n=7)$

Cirurgia de epilepsia prévia $(n=12)$

Dupla lesão epileptogênica $(n=10)$

Sem laudo de VEEG $(n=13)$

IRM normal $(n=17)$

Lesão temporal mesial $(n=26)$

Estado de mal epiléptico $(\mathrm{n}=1)$

\section{2 pacientes elegíveis}

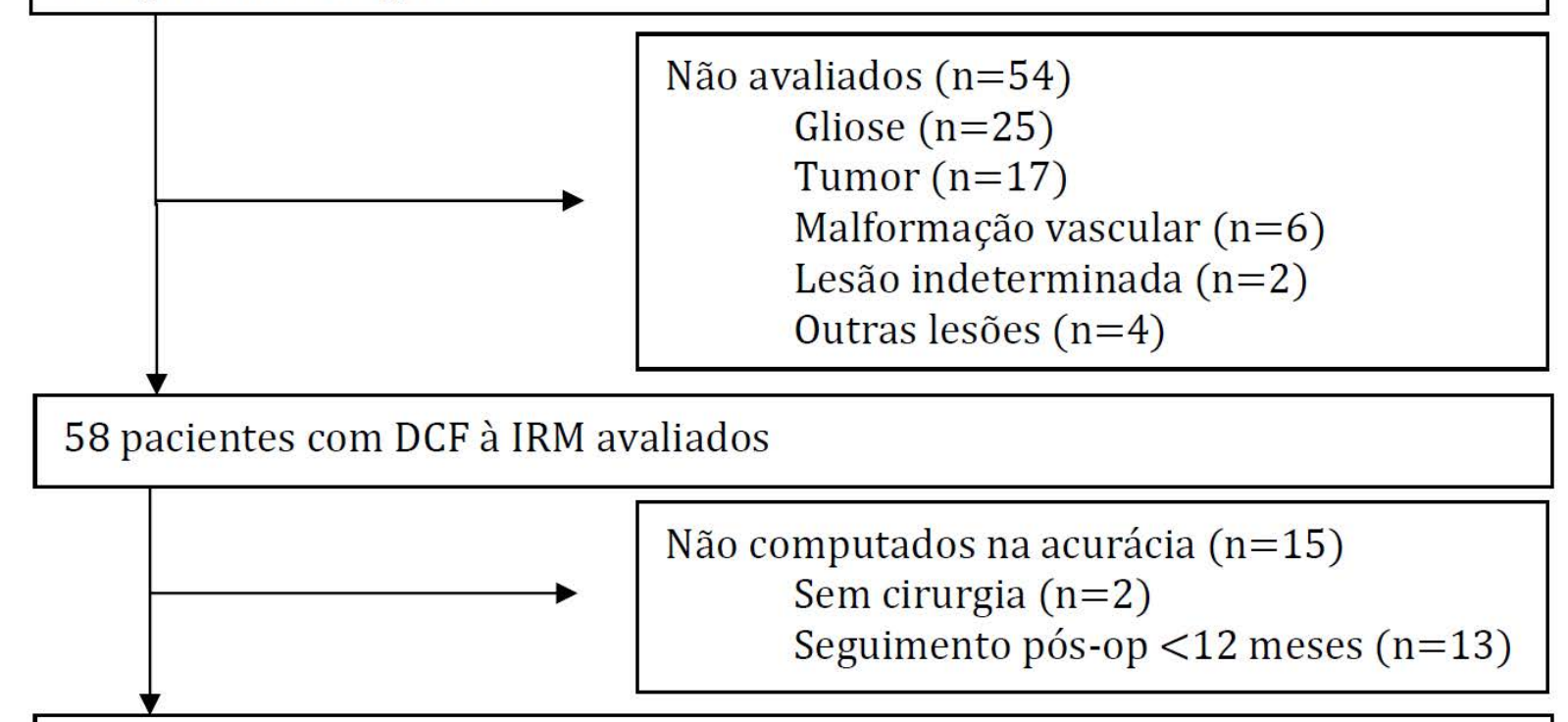

43 pacientes com DCF à IRM foram considerados para avaliação de acurácia

Figura 3: Fluxo da amostra. VEEG: vídeoeletroencefalografia; DCF: displasia cortical focal; IRM: imagem de ressonância magnética de encéfalo; CIREP: Centro de Cirurgia de Epilepsia - HC USP Ribeirão. 


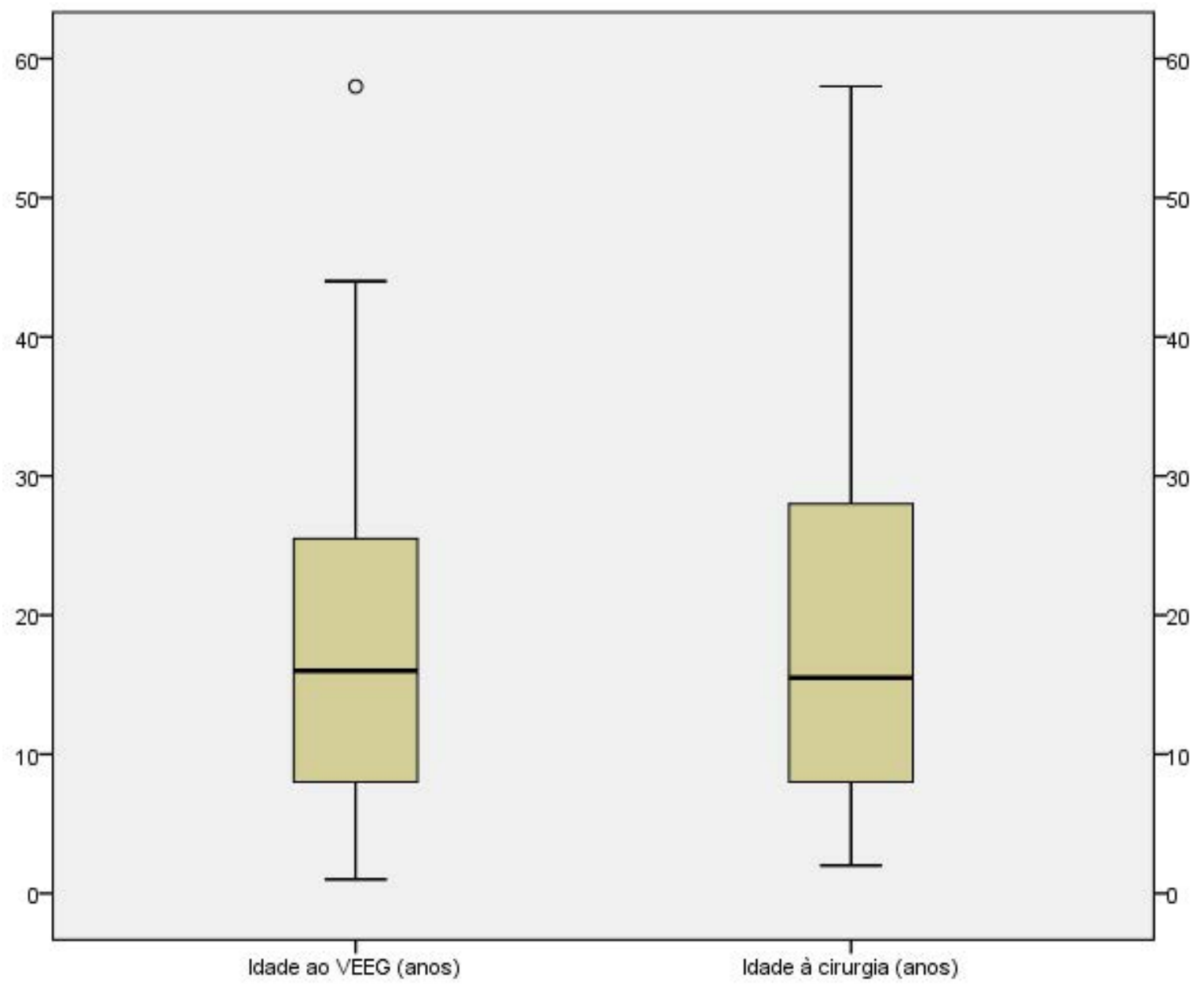

Figura 4: Distribuição das idades à VEEG e à cirurgia.

A duração mediana da epilepsia à VEEG foi de 8,5 anos, sendo a menor duração foi de 7 meses e a maior de 49 anos. A maioria dos pacientes apresentava entre 5 e 17 anos de doença à VEEG. À cirurgia, o tempo mediano de epilepsia foi de 10,5 anos, sendo a menor duração de 1 ano e a maior 49 anos. A maioria dos pacientes operados possuía entre 5 e 18 anos de duração da epilepsia (Figura 5).

O tempo de acompanhamento clínico após cirurgia foi de 2 a 8 anos na maioria dos casos, sendo o tempo mediano de 4 anos. O acompanhamento mais longo durou 16 anos.

A maior parte dos pacientes possuía imagens de ressonância magnética realizadas na máquina de 1,5 T. A partir de meados de 2007, com a introdução da máquina de 3,0 T os exames de epilepsia deixaram de ser realizados na máquina de 1,5 T (Tabela 1).

Tabela 1: Distribuição da amostra pela tecnologia de IRM.

\begin{tabular}{rrrrr}
\hline \hline & Frequência & Porcentagem & Porcentagem válida & Porcentagem acum. \\
\hline $1,5 \mathrm{~T}$ & 41 & 70,7 & 70,70 & 70,7 \\
$3,0 \mathrm{~T}$ & 17 & 29,3 & 29,3 & 100,0 \\
& 58 & 100,0 & 100,0 & \\
\hline \hline
\end{tabular}




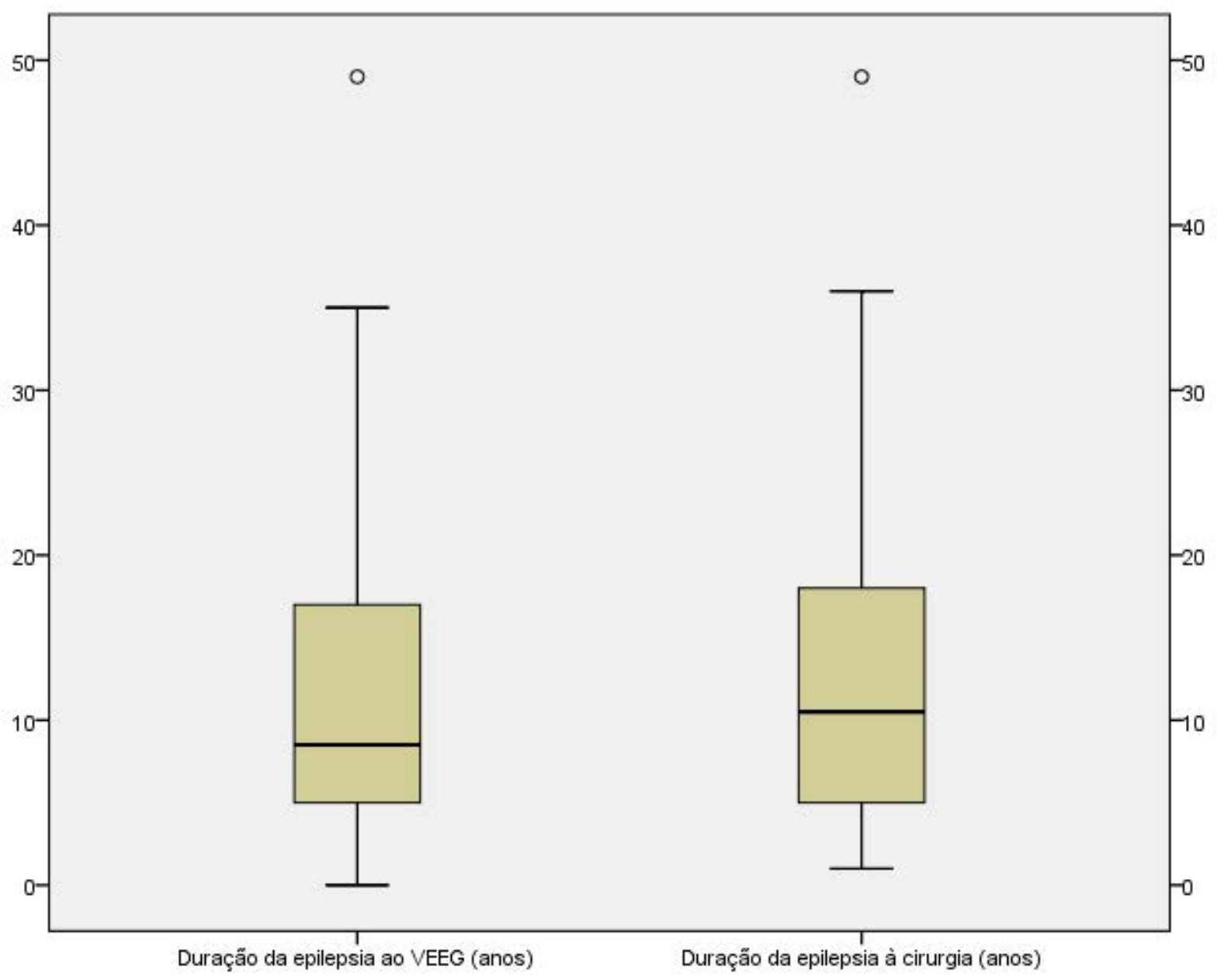

Figura 5: Duração da epilepsia à VEEG e à cirurgia.

Tabela 2: Distribuição da amostra pelo tipo de lesão à anatomia patológica.

\begin{tabular}{lrrrr}
\hline \hline & Frequência & Porcentagem & Porcentagem válida & Porcentagem acum. \\
\hline DCF & 51 & 87,9 & 91,1 & 91,1 \\
Gliose & 1 & 1,7 & 1,8 & 92,9 \\
Tumor & 2 & 3,4 & 3,6 & 96,4 \\
Indeterminado & 2 & 3,4 & 3,6 & 100,0 \\
\multirow{3}{*}{ Sem cirurgia } & 56 & 96,6 & 100,0 & \\
& 2 & 3,4 & & \\
\hline \hline
\end{tabular}

Todos os pacientes operados tiveram seu material ressecado submetido à análise da anatomia patológica. Em 91,1\% dos casos diagnosticados como DCF radiologicamente, houve confirmação patológica (Tabela 2).

Os pacientes apresentavam mais frequentemente a lesão epileptogênica na região frontal, seguida das regiões temporal neocortical e parietoccipital. Cerca de 10,3\% dos pacientes apresentavam lesões hemisféricas. O hemisfério esquerdo foi ligeiramente mais afetado (51,7\% dos casos) (Tabela 4). Em 18 casos, a lesão epileptogênica foi considerada 
como maior do que um lobo cerebral (Tabela 3).

Tabela 3: Distribuição da amostra pelo local da lesão epileptogênica (IRM).

\begin{tabular}{lrrrr}
\hline \hline & Frequência & Porcentagem & Porcentagem válida & Porcentagem acum. \\
\hline Frontal & 22 & 37,9 & 37,9 & 37,9 \\
Temporal & 14 & 24,1 & 24,1 & 62,1 \\
Insular & 1 & 1,7 & 1,7 & 63,8 \\
Central & 3 & 5,2 & 5,2 & 69,0 \\
Parietoccipital & 7 & 12,1 & 12,1 & 81,0 \\
Hemisférico & 6 & 10,3 & 10,3 & 91,4 \\
Frontocentral & 2 & 3,4 & 3,4 & 94,8 \\
TPO & 3 & 5,2 & 5,2 & 100,0 \\
& 58 & 100,0 & 100,0 & \\
\hline \hline
\end{tabular}

TPO: temporoparietoccipital.

Tabela 4: Distribuição da amostra pelo hemisfério da lesão epileptogênica (IRM).

\begin{tabular}{lrrrr}
\hline \hline & Frequência & Porcentagem & Porcentagem válida & Porcentagem acum. \\
\hline Esquerdo & 30 & 51,7 & 51,7 & 51,7 \\
Direito & 28 & 48,3 & 48,3 & 100,0 \\
& 58 & 100,0 & 100,0 & \\
\hline \hline
\end{tabular}

A zona de início ictal ao VEEG era mais frequentemente localizada na região frontal, seguida das regiões temporal neocortical e hemisférica. Houve dificuldade na localização da região de origem das crises em 8 pacientes (13,7\%), pois em 4 o padrão eletroencefalográfico foi difuso e em 4 não foi possível a lateralização hemisférica (Tabelas 5 e 6). Em 23 casos a zona de início ictal foi considerada como maior do que um lobo cerebral (Tabela 5).

Tabela 5: Distribuição da amostra pela região da zona de início ictal (VEEG).

\begin{tabular}{lrrrr}
\hline \hline & Frequência & Porcentagem & Porcentagem válida & Porcentagem acum. \\
\hline Frontal & 19 & 32,8 & 32,8 & 32,8 \\
Temporal & 12 & 20,7 & 20,7 & 53,4 \\
Insular & 1 & 1,7 & 1,7 & 55,2 \\
Central & 3 & 5,2 & 5,2 & 60,3 \\
Parietoccipital & 5 & 8,6 & 8,6 & 86,2 \\
Hemisférico & 10 & 17,2 & 17,2 & 93,1 \\
Difuso & 4 & 6,9 & 6,9 & 94,8 \\
Frontocentral & 1 & 1,7 & 1,7 & 96,6 \\
Frontotemporal & 1 & 1,7 & 1,7 & 100,0 \\
TPO & 2 & 3,4 & 3,4 & \\
& 58 & 100,0 & 100,0 &
\end{tabular}

TPO: temporoparietoccipital.

A zona irritativa de 18 pacientes (31\%) era conhecida antes da internação para VEEG, por meio da avaliação do EEG interictal ambulatorial. Foi observada uma grande 
Tabela 6: Distribuição da amostra pelo hemisfério da zona de início ictal (VEEG).

\begin{tabular}{lrrrr}
\hline \hline & Frequência & Porcentagem & Porcentagem válida & Porcentagem acum. \\
\hline Esquerdo & 26 & 44,8 & 44,8 & 44,8 \\
Direito & 24 & 41,4 & 41,4 & 86,2 \\
Não- & & & & \\
lateralizatório & 8 & 13,8 & 13,8 & 100,0 \\
& 58 & 100,0 & 100,0 & \\
\hline \hline
\end{tabular}

dispersão de resultados, ocorrendo principalmente descargas temporais e difusas. Em cerca de 22,2\% dos pacientes, o EEG não apresentou descargas epileptiformes (Tabela 7). Foi possível lateralizar as descargas em 44,4\% dos eletroencefalogramas interictais (Tabela 8).

Tabela 7: Localização da zona irritativa (EEG interictal).

\begin{tabular}{lrrrr}
\hline \hline & Frequência & Porcentagem & Porcentagem válida & Porcentagem acum. \\
\hline Frontal & 1 & 1,7 & 5,6 & 5,6 \\
Temporal & 4 & 6,9 & 22,2 & 27,8 \\
Central & 1 & 1,7 & 5,6 & 33,3 \\
Parietoccipital & 2 & 3,4 & 11,1 & 44,4 \\
Hemisférico & 2 & 3,4 & 11,1 & 55,6 \\
Difuso & 4 & 6,9 & 22,2 & 77,8 \\
Sem descargas & 4 & 6,9 & 22,2 & 100 \\
& 18 & 31,0 & 100,0 & \\
Sem EEG & 40 & 69,0 & & \\
& 58 & 100,0 & & \\
\hline \hline
\end{tabular}

Tabela 8: Lateralização da zona irritativa (EEG interictal).

\begin{tabular}{lrrrr}
\hline \hline & Frequência & Porcentagem & Porcentagem válida & Porcentagem acum. \\
\hline Esquerda & 3 & 5,2 & 16,7 & 16,7 \\
Direita & 5 & 8,6 & 27,8 & 44,4 \\
Bilateral & 6 & 10,3 & 33,3 & 77,8 \\
Sem descargas & 4 & 6,9 & 22,2 & 100,0 \\
& 18 & 31,0 & 100,0 & \\
Sem EEG & 40 & 69,0 & & \\
& 58 & 100,0 & & \\
\hline \hline
\end{tabular}

Cirurgia de epilepsia foi realizada em 56 pacientes (96,6\%). A região frontal foi alvo de ressecção em 35,7\% das cirurgias, sendo seguida pela região temporal neocortical (23,2\%) e parietoccipital (14,3\%) (Tabela 9). Em 73,2\% das cirurgias foi necessária investigação invasiva utilizando eletrocorticografia intraoperatória (ECoG; $50 \%$ das cirurgias) ou monitorização eletroencefalográfica crônica (23,2\% das cirurgias) (Tabela 10). Houve discreta preferência pela monitorização do hemisfério cerebral direito $(48,8 \%)$ sobre o esquerdo (46,3\%). Em apenas dois pacientes, houve monitorização bilateral (4,9\%) (Tabela 11). Estimulação cortical foi conduzida em 30,4\% das cirurgias (Tabela 12). 
Tabela 9: Localização da cirurgia.

\begin{tabular}{lrrrr}
\hline \hline & Frequência & Porcentagem & Porcentagem válida & Porcentagem acum. \\
\hline Frontal & 20 & 34,1 & 35,7 & 35,7 \\
Temporal & 13 & 22,4 & 23,2 & 58,9 \\
Central & 3 & 5,2 & 5,4 & 64,3 \\
Parietoccipital & 8 & 13,8 & 14,3 & 78,6 \\
Hemisférico & 6 & 10,3 & 10,7 & 89,3 \\
Frontocentral & 3 & 5,2 & 5,4 & 94,6 \\
Frontotemporal & 2 & 3,4 & 3,6 & 98,2 \\
TPO & 1 & 1,7 & 1,8 & 100,0 \\
& 56 & 96,6 & 100,0 & \\
Sem cirurgia & 2 & 3,4 & & \\
& 58 & 100,0 & &
\end{tabular}

TPO: temporoparietoccipital.

Tabela 10: Realização de investigação invasiva.

\begin{tabular}{lrrrr}
\hline \hline & Frequência & Porcentagem & Porcentagem válida & Porcentagem acum. \\
\hline Intraoperatória & 28 & 48,2 & 50 & 50 \\
Crônica & 13 & 22,4 & 23,2 & 73,2 \\
Sem invasivos & 15 & 25,8 & 26,8 & 100,00 \\
& 56 & 96,6 & 100,0 & \\
Sem cirurgia & 2 & 3,4 & & \\
& 58 & 100,0 & & \\
\hline \hline
\end{tabular}

Tabela 11: Lateralização dos eletrodos invasivos.

\begin{tabular}{lrrrr}
\hline \hline & Frequência & Porcentagem & Porcentagem válida & Porcentagem acum. \\
\hline Esquerda & 19 & 32,8 & 46,3 & 46,3 \\
Direita & 20 & 34,5 & 48,8 & 95,1 \\
Bilateral & 2 & 3,4 & 4,9 & 100,0 \\
& 41 & 70,7 & 100,0 & \\
Sem invasivos & 15 & 25,9 & & \\
Sem cirurgia & 2 & 3,4 & & \\
& 58 & 100,0 & & \\
\hline \hline
\end{tabular}

Era conhecido o desfecho pós-operatório de 43 pacientes. Em todos os casos, no mínimo um ano de acompanhamento pós-operatório foi considerado. Em média, o acompanhamento pós-operatório teve duração de 84 meses. Cerca de 60,5\% dos pacientes acompanhados foram seguidos por mais de 5 anos (Tabela 13). Houve predomínio de desfecho positivo (vida de livre de crises; Engel I), que ocorreu em 55,8\% dos pacientes sobre o desfecho negativo (persistência de crises; não-Engel I) em 44,9\% dos casos (Tabela $14)$. 
Tabela 12: Realização de estimulação cortical.

\begin{tabular}{lrrrr}
\hline \hline & Frequência & Porcentagem & Porcentagem válida & Porcentagem acum. \\
\hline Intraoperatória & 11 & 18,9 & 19,6 & 19,6 \\
Crônica & 6 & 10,3 & 10,7 & 30,4 \\
Sem & & & & \\
estimulação & 39 & 67,2 & 69,6 & 100,0 \\
& 56 & 96,6 & 100,0 & \\
Sem cirurgia & 2 & 3,4 & & \\
& 58 & 100,0 & & \\
\hline \hline
\end{tabular}

Tabela 13: Duração do acompanhamento pós-operatório.

\begin{tabular}{lrrrr}
\hline \hline & Frequência & Porcentagem & Porcentagem válida & Porcentagem acum. \\
\hline 12 a 24 meses & 4 & 6,9 & 9,3 & 9,3 \\
24 a 60 meses & 13 & 22,4 & 30,2 & 39,5 \\
$>$ 60 meses & 26 & 44,8 & 60,5 & 100,0 \\
& 43 & 74,1 & 100,0 & \\
Sem cirurgia & 2 & 3,4 & & \\
Sem acompa- & & & & \\
nhamento ou & 13 & 22,5 & & \\
$<12$ meses & 58 & 100,0 & & \\
& & & & \\
\hline \hline
\end{tabular}

Tabela 14: Desfecho pós-cirúrgico (>12 meses de acompanhamento).

\begin{tabular}{lrrrr}
\hline \hline & Frequência & Porcentagem & Porcentagem válida & Porcentagem acum. \\
\hline Engel I & 24 & 41,4 & 55,8 & 55,8 \\
Não-Engel I & 19 & 32,7 & 44,9 & 100,0 \\
& 43 & 74,1 & 100,0 & \\
Sem classificação & 13 & 22,5 & & \\
Sem cirurgia & 2 & 3,4 & & \\
& 58 & 100,0 & & \\
\hline \hline
\end{tabular}

\subsection{Análise da convergência entre a VEEG e a IRM}

Todos os pacientes foram submetidos ao exame de imagem por ressonância magnética e à monitorização por vídeoeletroencefalografia como protocolo mínimo para investigação de cirurgia de epilepsia. Todos os casos foram discutidos em reunião clínica, com o objetivo de determinar se havia indicação cirúrgica, e em caso positivo, em que local seria realizada a cirurgia. Além disso, era determinado se havia necessidade ou não de monitorização invasiva crônica ou eletrocorticografia intraoperatória, além de estimulação cortical quando a área a ser ressecada estivesse próximas a regiões eloquentes.

Foi identificado exclusivamente um lobo como região de início ictal em 35 pacientes $(60 \%)$ e exclusivamente um lobo contendo a lesão epileptogênica em 40 pacientes (69\%). Houve 23 pacientes em que a lesão epileptogênica e/ou a zona de início ictal foi considerada 
maior do que um lobo cerebral, podendo este envolvimento ser frontocentral, frontotemporal, parietoccipital, temporoparietoccipital ou hemisférico.

Ao se analisar a relação da região de início ictal observada ao VEEG com o local da lesão epileptogênica observada à IRM, obtém-se uma convergência (kappa de Cohen; K) de 0,599 ( $<<0,01 ;$ IC 95\% 0,468-0,730), que é considerada uma concordância moderada (Tabela 15).

Em $60,7 \%$ dos casos operados, o local da cirurgia coincidiu com o local da zona de início ictal determinada pelo laudo de VEEG (Tabela 16). Em 82,1\% dos casos operados, o local da cirurgia coincidiu com o local da DCF identificada à IRM (Tabela 17). Ao se distribuir as regiões de início ictal à VEEG e da lesão epileptogênica à IRM, em relação ao local onde foi realizada a cirurgia (padrão de referência), observa-se que a concordância da VEEG $(\mathrm{K}=0,566, \mathrm{p}<0,01$; IC $95 \%$ 0,433-0,759) pode ser considerada moderada, enquanto a da IRM $(\mathrm{K}=0,798, \mathrm{p}<0,01$; IC 95\% 0,687-0,909) pode ser considerada substancial.

\subsection{Análise da acurácia da VEEG e da IRM}

Para avaliação da acurácia diagnóstica da VEEG e da IRM, foram selecionados da população estudada aqueles casos que foram submetidos à cirurgia de epilepsia, e que possuíam acompanhamento pós-operatório superior a 12 meses. Esta escolha seguiu orientações da literatura sobre formas de melhorar as avaliações de acurácia orientadas à cirurgia de epilepsia (BURCH et al., 2012), em que se sugere que a complementação do padrão de referência (decisão clínica da equipe multidisciplinar) com desfecho clínico pós-operatório melhora a avaliação da acurácia dos exames em questão.

Desta forma, 43 pacientes atendiam aos critérios para avaliação de acurácia. Para que tal avaliação ocorra, é necessário que para cada exame em teste seja elaborada uma tabela cruzada. Os dados concordantes são aqueles em que o teste em questão tenha coincidido com o local da cirurgia. Sugere-se que para avaliação dos testes aplicados à cirurgia de epilepsia, a distribuição dos dados numa tabela cruzada modelo seja conforme convenção (BURCH et al., 2012) (Tabela 18).

Para cada um dos testes em questão foi confeccionada uma tabela cruzada, seguindo o modelo. Dela, podem ser obtidas informações críticas para a determinação da acurácia diagnóstica, como sensibilidade, especificidade, valor preditivo positivo, valor preditivo negativo e razões de verossimilhança.

A sensibilidade foi calculada pela razão $\mathrm{A} /(\mathrm{A}+\mathrm{C})$; a especificidade pela razão $\mathrm{D} /(\mathrm{D}+\mathrm{B})$; o valor preditivo positivo pela razão $\mathrm{A} /(\mathrm{A}+\mathrm{B})$; o valor preditivo negativo pela razão $\mathrm{D} /(\mathrm{C}+\mathrm{D})$; a razão da verossimilhança positiva como sensibilidade/(1-especificidade) e a razão da verossimilhança negativa como (1-sensibilidade)/especificidade. 


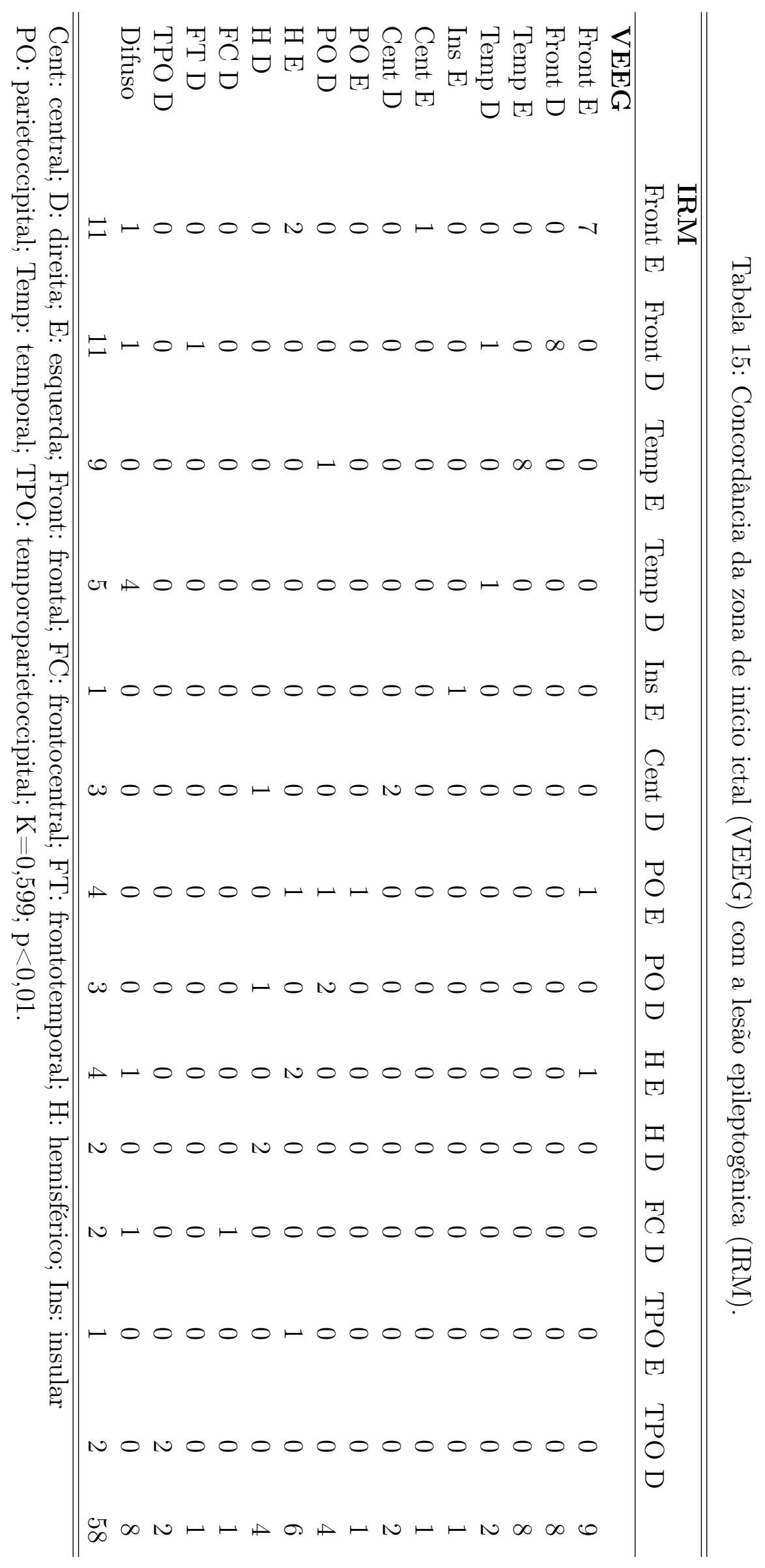




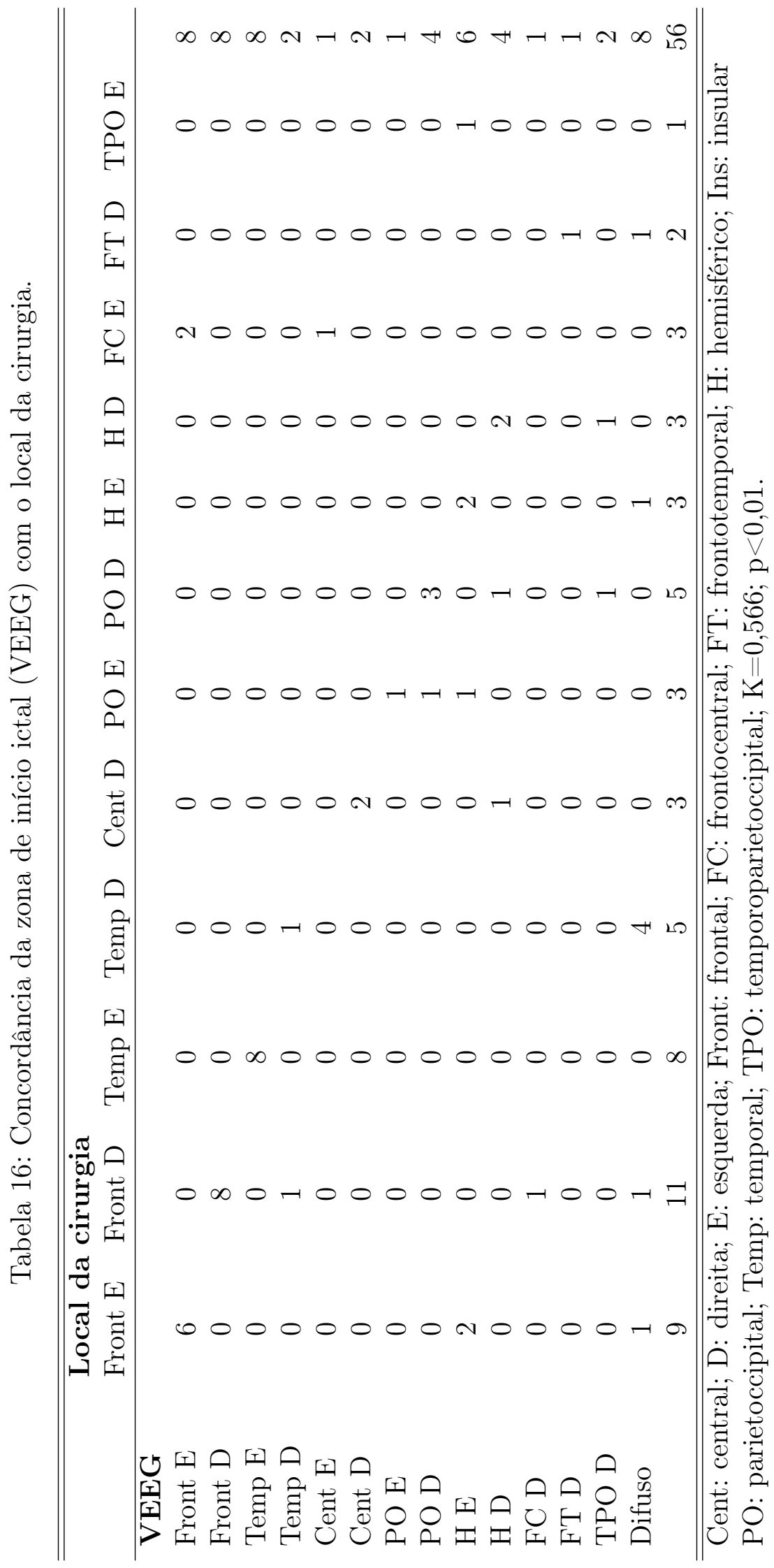




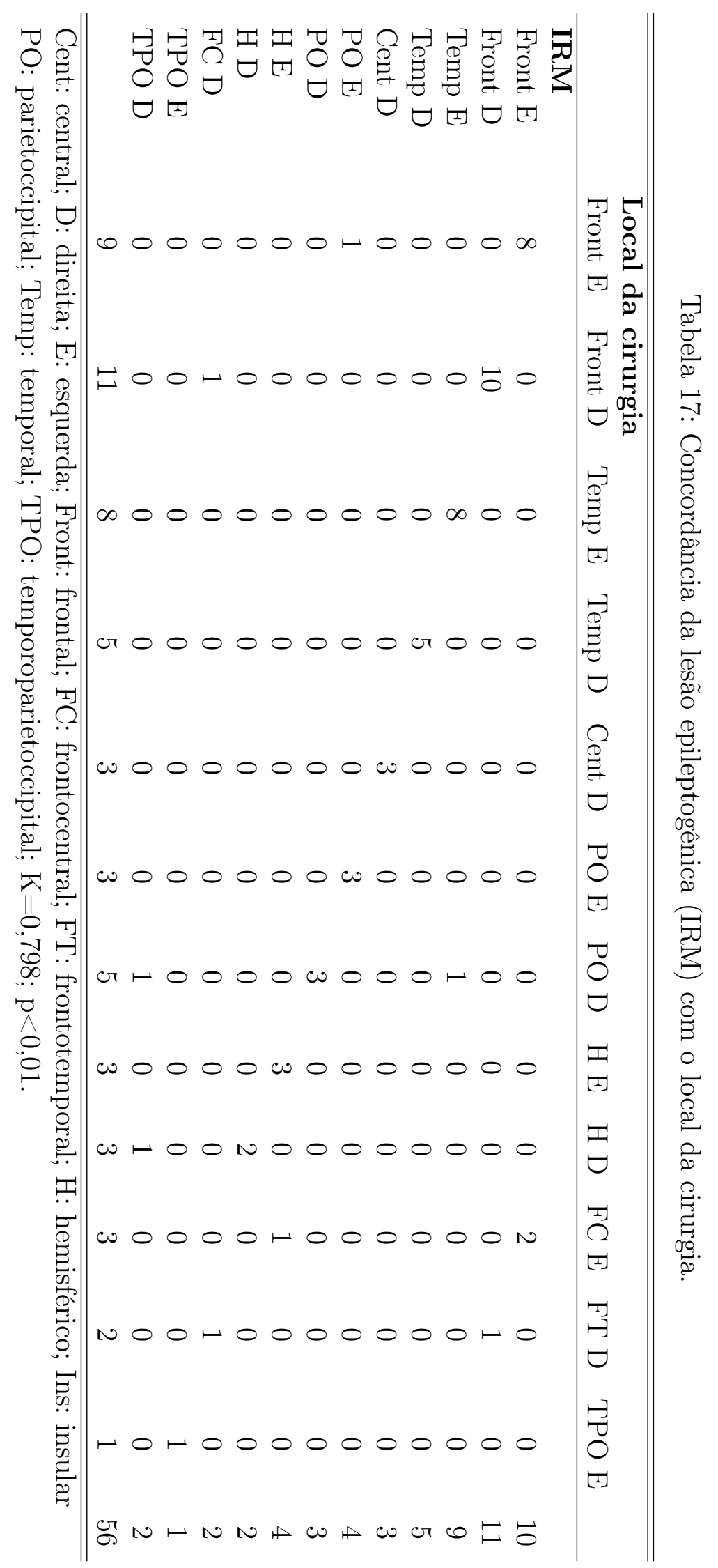


Tabela 18: Tabela cruzada modelo.

\begin{tabular}{lcc}
\hline \hline & Desfecho pós-operatório bom & Desfecho pós-operatório ruim \\
\hline Dados concordantes & $\mathrm{A}$ & $\mathrm{B}$ \\
Dados discordantes & $\mathrm{C}$ & $\mathrm{D}$ \\
\hline \hline
\end{tabular}

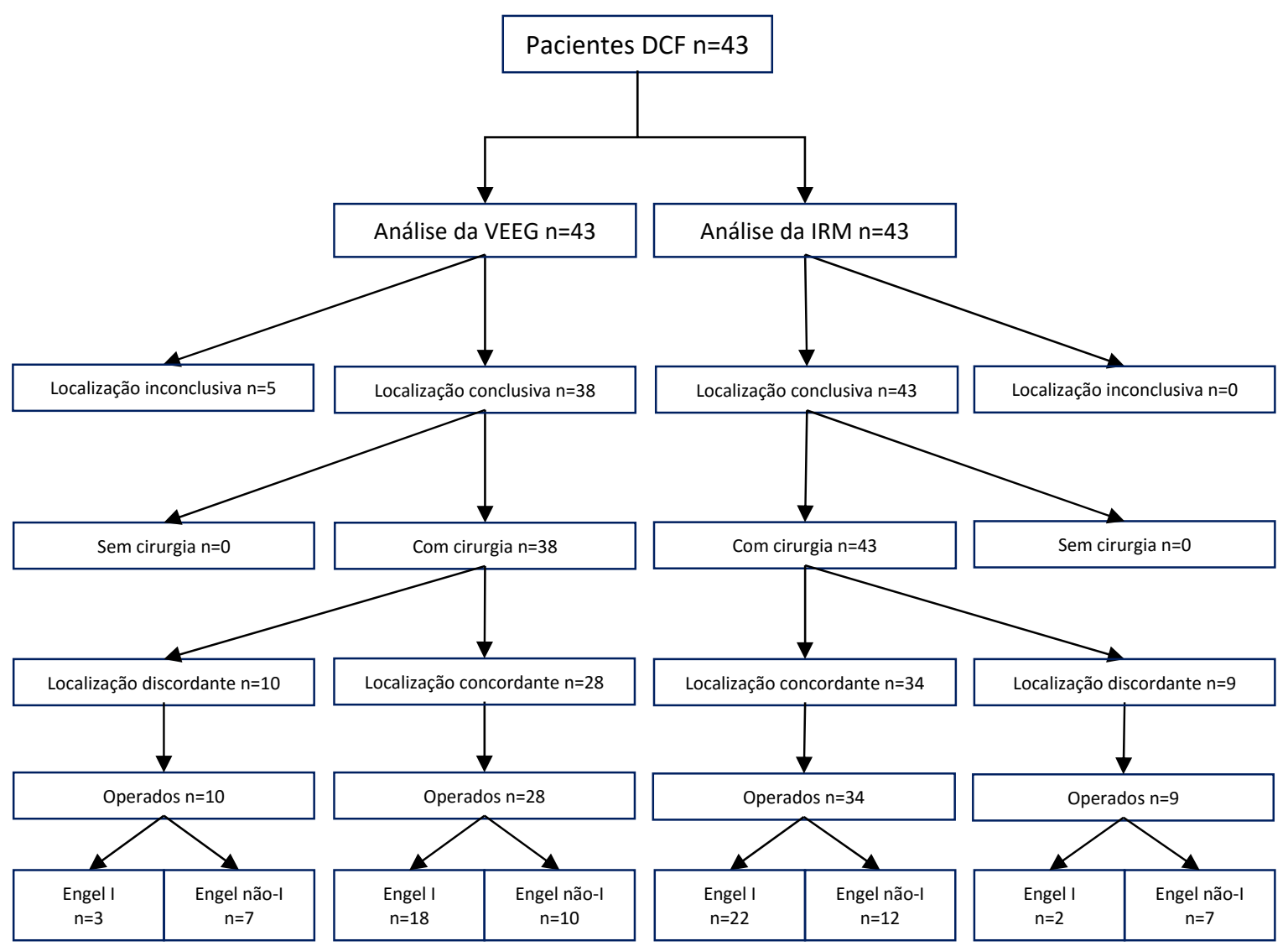

Figura 6: Fluxo de resultados para cálculos de acurácia. DCF: displasia cortical focal; IRM: imagem de ressonância magnética; VEEG: videoeletroencefalografia.

No estudo, foram considerados como dados concordantes aqueles pacientes em que o resultado do exame-índice (VEEG ou IRM) coincidia com o local em que foi realizada a cirurgia. Dados discordantes eram aqueles em que o exame-índice não coincidia com o local da cirurgia.

Desfechos pós-operatórios foram considerados bons quando a classificação pósoperatória era Engel I (vida livre de crises), e ruins quando não era Engel I (vida não-livre de crises).

As tabelas cruzadas foram povoadas baseadas no fluxo dos resultados (Figura 6).

Sendo assim, os dados são aplicados à tabela cruzada da distribuição dos casos para VEEG (observando-se que cinco casos foram excluídos pois sua localização era inconclusiva) (Tabela 19). 
Tabela 19: Tabela cruzada para VEEG.

\begin{tabular}{lccc}
\hline \hline & Engel I & Não Engel I & \\
\hline Dados concordantes & 18 & 10 & 28 \\
Dados discordantes & 3 & 7 & 10 \\
& 21 & 17 & 38 \\
\hline \hline $\mathrm{N}=$ pacientes. & &
\end{tabular}

Aplicando os números da tabela cruzada nas equações de acurácia, obtém-se os resultados do desempenho diagnóstico da VEEG (Tabela 20).

Tabela 20: Desempenho diagnóstico da VEEG.

\begin{tabular}{lccc}
\hline \hline & & \multicolumn{2}{c}{ IC 95\% } \\
& Valor & Limite inf. & Limite sup. \\
\hline Sensibilidade & 0,857 & 0,626 & 0,962 \\
Especificidade & 0,411 & 0,194 & 0,665 \\
Valor preditivo positivo & 0,642 & 0,441 & 0,806 \\
Valor preditivo negativo & 0,700 & 0,353 & 0,919 \\
Razão da verossimilhança positiva & 1,457 & 0,943 & 2,249 \\
Razão da verossimilhança negativa & 0,346 & 0,103 & 1,166 \\
\hline \hline
\end{tabular}

IC: intervalo de confiança.

Da mesma forma, procede-se à distribuição dos casos para a tabela cruzada da IRM (Tabela 21).

Tabela 21: Tabela cruzada para IRM.

\begin{tabular}{lccc}
\hline \hline & Engel I & Não Engel I & \\
\hline Dados concordantes & 22 & 12 & 34 \\
Dados discordantes & 2 & 7 & 9 \\
& 24 & 19 & 43 \\
\hline \hline
\end{tabular}

$\mathrm{N}=$ pacientes.

Finalmente, aplicando os números nas equações de acurácia, obtém-se os resultados do desempenho diagnóstico da IRM (Tabela 22).

\subsection{Comparação da acurácia da VEEG e da IRM}

Quando se deseja comparar dois testes diagnósticos, para verificar se há superioridade de um sobre o outro ou mesmo se ambos são equivalentes, devem ser utilizados métodos estatísticos (HAWASS, 1997). Foram utilizados neste estudo a comparação entre sensibilidade e especificidade, a área sob a curva ROC (AUCROC) e o índice de Youden (J). É importante, em cada cálculo e para cada teste, que seja gerado um valor, estabelecido seu intervalo de confiança e a diferença entre os valores seja submetida à análise estatística para determinação se há ou não significância estatística. 
Tabela 22: Desempenho diagnóstico da IRM.

\begin{tabular}{lccc}
\hline \hline & & \multicolumn{2}{c}{ IC 95\% } \\
& Valor & Limite inf. & Limite sup. \\
\hline Sensibilidade & 0,916 & 0,715 & 0,985 \\
Especificidade & 0,368 & 0,172 & 0,613 \\
Valor preditivo positivo & 0,647 & 0,464 & 0,796 \\
Valor preditivo negativo & 0,777 & 0,401 & 0,960 \\
Razão da verossimilhança positiva & 1,451 & 1,008 & 2,088 \\
Razão da verossimilhança negativa & 0,226 & 0,050 & 1,017 \\
\hline \hline
\end{tabular}

IC: intervalo de confiança.

Ao comparar os índices de sensibilidade e especificidade, é apropriado que os dados sejam submetidos à avaliação adequada (HAWASS, 1997) devido a uma série de limitações que ocorrem ao se confrontar os dados diretamente. De forma geral, tabelas cruzadas não apresentam informação sobre o quanto os testes em avaliação concordam entre si, nem quanto não concordam. Esta análise é crucial para a avaliação de testes diagnósticos numa amostra pareada. No estudo atual, a diferença entre as sensibilidades foi de 5,9\% favorecendo a IRM, e a diferença entre as especificidades foi de 4,3\% favorecendo a VEEG. Deve-se proceder à análise estatística para verificar se estas diferenças são estatisticamente significantes.

Para resolver esta questão, torna-se necessária a construção de uma nova tabela cruzada, que reflita o quanto os testes concordam e discordam entre si, tendo como base o desfecho pós-operatório. Assim, elaboram-se duas tabelas: uma que inclua somente os pacientes livre de crises epilépticas e outra que compreenda somente os pacientes não-livres de crises epilépticas. Os valores das caselas corresponderão aos pacientes em que houve concordância do exame com o local da cirurgia (valores positivos) ou em que não houve concordância do exame com o local da cirurgia (valores negativos).

A tabela que corresponde a uma vida livre de crises pode ser interpretada como uma tabela de comparação entre a sensibilidade dos dois métodos (Tabela 23), enquanto que a tabela cruzada da concordância dos testes nos pacientes em que não houve vida livre de crises após cirurgia pode ser interpretada como uma tabela de comparação das especificidades dos dois métodos (Tabela 24).

Tabela 23: Comparação das sensibilidades dos dois métodos (pacientes livres de crises).

\begin{tabular}{ccccc}
\hline \hline & & \multicolumn{3}{c}{ IRM } \\
\hline \multirow{3}{*}{ VEEG } & Positiva & Negativa \\
& Negativa & 17 & 1 & 18 \\
& & 5 & 1 & 6 \\
& & 22 & 2 & 24 \\
\hline \hline $\mathrm{p}=0,219$ (McNemar). & \multicolumn{4}{c}{}
\end{tabular}

$\mathrm{p}=0,219$ (McNemar). 
Tabela 24: Comparação das especificidades dos dois métodos (pacientes não-livres de crises).

\begin{tabular}{ccccc}
\hline \hline & & \multicolumn{3}{c}{ IRM } \\
\hline \multirow{3}{*}{ VEEG } & & Positiva & Negativa \\
& Positiva & 8 & 2 & 10 \\
& Negativa & 4 & 5 & 9 \\
& & 12 & 7 & 19 \\
\hline \hline $\mathrm{p}=0,687$ (McNemar). & \multicolumn{3}{c}{}
\end{tabular}

Prosseguindo na análise, é necessário que seja calculado se as diferenças entre as sensibilidades e especificidades observadas possuem significância estatística. Para isso, é preciso que sejam destacados das tabelas os valores em que os testes foram divergentes (um positivo e outro negativo), uma vez que a diferença de desempenho dos testes não pode ser comparada quando os resultados são convergentes (HAWASS, 1997). A hipótese nula é que os valores divergentes não possuem diferença, e a hipótese alternativa é que os valores divergentes possuem significância estatística.

Observa-se que não foi encontrada diferença significativa entre o desempenho diagnóstico dos dois testes tanto na população livre de crises (sensibilidade) quanto na população não-livre de crises (especificidade) (Tabelas 23 e 24).

Além disso, é preciso que se saiba o erro padrão da diferença entre as proporções (S.E.), para que se estime os intervalos de confiança necessários para estabelecer a magnitude da diferença (HAWASS, 1997):

$$
S . E ._{(p 1-p 2)}=\frac{1}{n} \sqrt{b+c-\frac{(b-c)^{2}}{n}}
$$

Pela tabela de sensibilidade, obtém-se:

$$
\begin{gathered}
S . E \cdot_{\operatorname{Sens}(p 1-p 2)}=\frac{1}{n} \sqrt{b+c-\frac{(b-c)^{2}}{n}}=\frac{1}{24} \sqrt{1+5-\frac{(1-5)^{2}}{24}} \\
=0,112 \sqrt{1+5-\frac{16}{24}}=0,112 \sqrt{1+5-0,666}=0,112 \sqrt{5,334} \\
=0,258
\end{gathered}
$$

Então, sabendo-se que a sensibilidade previamente descrita é de 0,916 para a IRM e de 0,857 para a VEEG, tem-se:

$$
\begin{gathered}
I C 95_{\text {Sens }}=\left(\text { Sens }_{I R M}-\text { Sens }_{V E E G}\right) \pm 1,96\left(\text { S.E. } \cdot_{\text {Sens }(p 1-p 2)}\right) \\
=(0,916-0,857) \pm 1,96(0,258)= \\
=0,059 \pm 0,505
\end{gathered}
$$


Deste modo, pode-se afirmar que o intervalo de confiança de $95 \%$ para a diferença entre as sensibilidades é de $-44,6$ a $+56,4 \%$. Como o valor passa pelo zero, pode-se afirmar que não há diferença estatisticamente significativa entre os valores de sensibilidade dos dois exames (Tabela 25).

Tabela 25: Diferenças no desempenho diagnóstico da VEEG e da IRM.

\begin{tabular}{lcccc}
\hline \hline & & & \multicolumn{2}{c}{ IC 95\% } \\
\hline & Vantagem & Diferença & Limite inf. & Limite sup. \\
Sensibilidade & IRM & $5,9 \%$ & $-44,6 \%$ & $+56,4 \%$ \\
Especificidade & VEEG & $4,3 \%$ & $-20,6 \%$ & $+28,8 \%$ \\
\hline \hline
\end{tabular}

Pela tabela de especificidade, obtém-se:

$$
\begin{gathered}
S . E \cdot E s p(p 1-p 2)=\frac{1}{n} \sqrt{b+c-\frac{(b-c)^{2}}{n}}=\frac{1}{19} \sqrt{2+4-\frac{(2-4)^{2}}{19}} \\
=0,052 \sqrt{2+4-\frac{4}{19}}=0,052 \sqrt{2+4-0,210}=0,052 \sqrt{5,79} \\
=0,125
\end{gathered}
$$

Então, sabendo-se que a especificidade previamente descrita é de 0,411 para a VEEG e de 0,368 para a IRM, tem-se:

$$
\begin{gathered}
I C 95_{E s p}=\left(E s p_{V E E G}-E s p_{I R M} \pm 1,96(S \cdot E \cdot E s p(p 1-p 2))\right. \\
=(0,411-0,368) \pm 1,96(0,125) \\
=0,043 \pm 0,245
\end{gathered}
$$

Deste modo, pode-se afirmar que o intervalo de confiança de $95 \%$ para a diferença entre as especificidades é de $-20,2$ a $+28,8 \%$. Como o valor também passa pelo zero, pode-se afirmar que também não há diferença estatisticamente significativa entre os valores de especificidade dos dois exames (Tabela 25).

A AUCROC é uma medida global da acurácia diagnóstica, traduzido da sigla em inglês "area under the curve receiver-operating characteristic". Ele é limitado em sua informação sobre aspectos particulares do teste, como sensibilidade e especificidade. Porém, ao comparar a área sob duas curvas ROC é possível estimar qual dos dois testes é mais adequado para avaliar duas condições de interesse, como livre de crises e não-livre de crises epilépticas. Originalmente, tal método foi utilizado para avaliar se operadores de radar conseguiriam distinguir sinais observados na tela como amigos ou inimigos, portanto o nome em inglês ROC (receiver-operating characteristic). Deve ser ressaltado que esta comparação deve utilizar valores numéricos, e não análise intuitiva ou visual do gráfico (ZWEIG; CAMPBELL, 1993; OBUCHOWSKI; LIEBER; WIANS, 2004). 


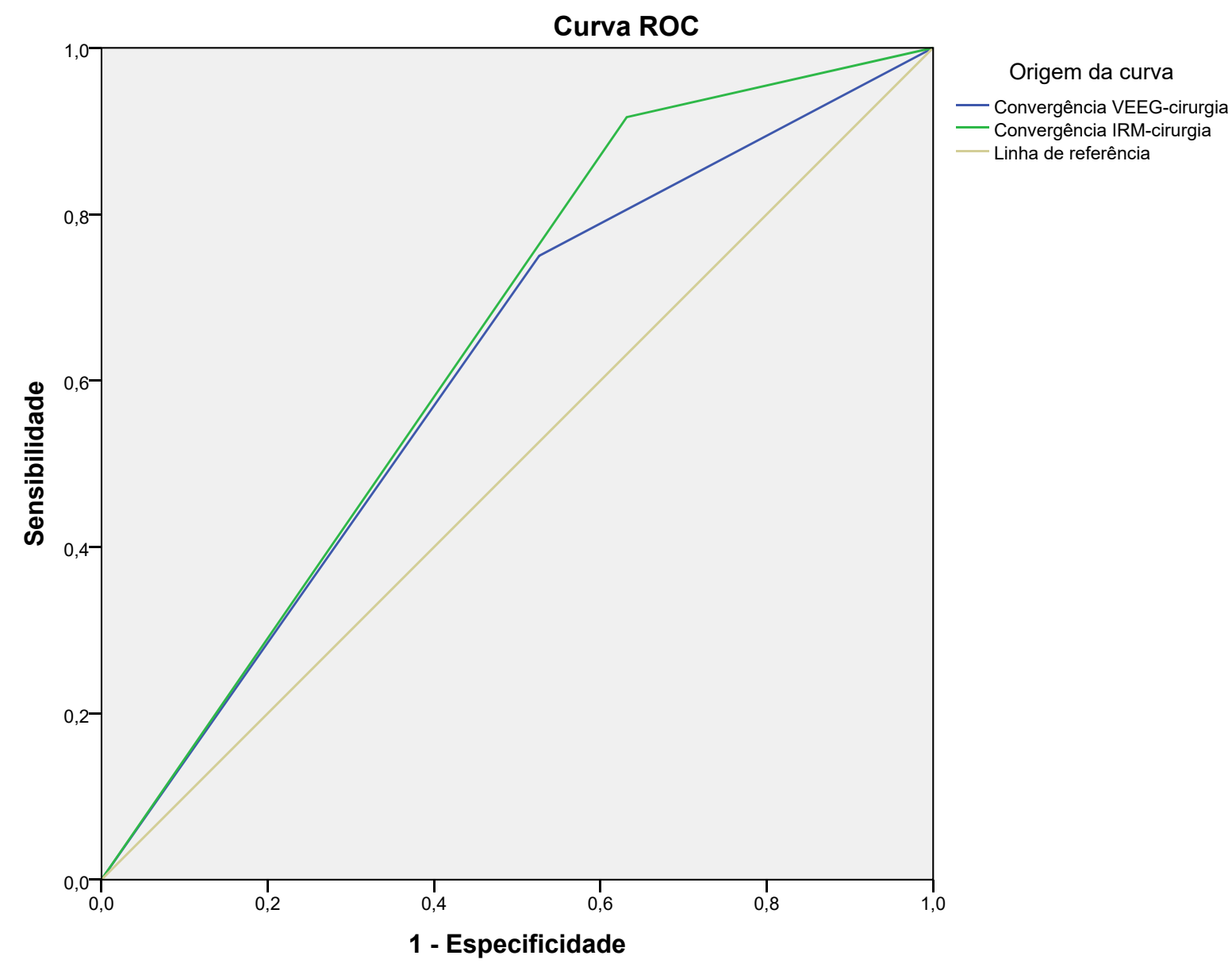

Figura 7: Curvas ROC (receiver-operating characteristic).

Dessa forma, deve ser aplicado o teste $t$ de amostras pareadas aos valores da AUCROC obtidos para determinar se há significância na diferença entre duas áreas (GLANTZ, 2005). Assume-se que a hipótese nula é quando não existe diferença entre os dois testes e que a hipótese alternativa é quando existe sim diferença entre os dois testes.

Aplicando os dados de acurácia da VEEG e da IRM para realização da curva ROC, obtém-se o gráfico (Figura 7) e áreas sob a curva (Tabela 26).

Tabela 26: Áreas sob as curvas ROC.

\begin{tabular}{cccccc}
\hline \hline & & & \multicolumn{2}{c}{ IC 95\% } \\
\hline $\begin{array}{c}\text { Convergência } \\
\text { IRM-cirurgia }\end{array}$ & 0,643 & 0,088 & 0,112 & 0,471 & 0,814 \\
$\begin{array}{c}\text { Convergência } \\
\text { VEEG-cirurgia }\end{array}$ & 0,612 & 0,088 & 0,212 & 0,439 & 0,784 \\
\hline \hline
\end{tabular}

Observa-se que a diferença entre as áreas é de 0,031 favorecendo a IRM. Para determinar se esta diferença possui significância estatística, inicialmente aplica-se o teste de correlação de Pearson (ZWEIG; CAMPBELL, 1993) (Tabela 27): 
Tabela 27: Coeficiente de correlação: diferença das áreas sob as curvas ROC.

\begin{tabular}{ccc}
\hline \hline & & Convergência VEEG-cirurgia \\
\hline Convergência IRM-cirurgia & Correlação de Pearson (r) & 0,343 \\
& Signif. (2 extremidades) & 0,024 \\
& $\mathrm{~N}$ & 43 \\
\hline \hline
\end{tabular}

Em seguida, aplica-se a variável r obtida acima na equação abaixo (HANLEY; MCNEIL, 1983):

$$
\begin{gathered}
\text { S.E.dif } \left.f_{A U C}=\sqrt{(S . E \cdot I R M)^{2}+(S . E \cdot V E E G)^{2}-2 r\left(S . E_{I R M}\right)(S . E \cdot V E E G}\right) \\
=\sqrt{(0,88)^{2}+(0,88)^{2}-2 r(0,88)(0,88)} \\
=\sqrt{0,7744+0,7744-2 r(0,7744)} \\
=\sqrt{0,7744+0,7744-2(0,343)(0,7744)} \\
=\sqrt{0,7744+0,7744-0,5312} \\
=\sqrt{1,0117} \\
=1,008
\end{gathered}
$$

O erro padrão da diferença encontrado pode, agora, ser aplicado ao teste t para medidas pareadas abaixo:

$$
\begin{gathered}
t=\frac{\text { diferença entre as áreas }}{\text { erro padrão da diferença }}=\frac{0,643-0,612}{1,008} \\
=\frac{0,031}{1,008}=0,030
\end{gathered}
$$

Como o valor esperado para t em um teste com $\mathrm{v}=42$ ([n-1] graus de liberdade) para um erro alfa de $5 \%$ seria no mínimo 2,018, pode-se assumir que o resultado de $\mathrm{t}=0,030$ encontrado não possui significância estatística (GLANTZ, 2005). Conclui-se, portanto, que não se pode rejeitar a hipótese nula. Deesa forma, os dois exames não possuem diferença estatisticamente significante na acurácia diagnóstica pelo método de AUCROC.

Outro método empregado na comparação entre testes diagnósticos é o índice de Youden (YOUDEN, 1950). Um dos métodos mais antigos para medida de acurácia diagnóstica, este índice também é uma medida global de desempenho, usado para avaliação do poder discriminativo de um procedimento diagnóstico e para comparação com outros testes. Seu resultado varia entre zero e um, sendo valores próximos a um considerados 
melhores. O índice de Youden (J) é calculado utilizando-se como base as tabelas cruzadas da VEEG e da IRM, da seguinte forma:

$$
J=\frac{a d-b c}{(a+c)(b+d)}
$$

Substituindo os valores da equação pelos valores obtidos nas tabelas cruzadas, temos o seguinte valor de J para a VEEG:

$$
\begin{gathered}
J_{V E E G}=\frac{(18)(7)-(10)(3)}{(18+3)(10+7)}=\frac{(126)-(30)}{(21)(17)} \\
=\frac{96}{357}=0,268
\end{gathered}
$$

Repetindo-se o procedimento e aplicando-se os valores da tabela cruzada da IRM obtemos:

$$
\begin{gathered}
J_{I R M}=\frac{(22)(7)-(12)(2)}{(22+2)(12+7)}=\frac{(154)-(24)}{(24)(19)} \\
=\frac{130}{456}=0,285
\end{gathered}
$$

Percebe-se uma diferença de 0,017 no índice de Youden, favorecendo a IRM. Da mesma forma que no método anterior, deve-se aplicar o teste t para determinar se há significância estatística na diferença entre os valores encontrados. Neste caso, deve-se calcular inicialmente o erro padrão de cada exame (YOUDEN, 1950). Dessa forma, obtémse para a VEEG:

$$
\begin{gathered}
\text { S.E.Jveeg }=\sqrt{\frac{a c}{(a+c)^{3}}+\frac{b d}{(b+d)^{3}}}=\sqrt{\frac{(18)(3)}{(21)^{3}}+\frac{(10)(7)}{(17)^{3}}} \\
=\sqrt{\frac{54}{9261}+\frac{70}{4913}}=\sqrt{0,005+0,014} \\
=\sqrt{0,019}=0,137
\end{gathered}
$$

Repete-se o mesmo cálculo para a IRM:

$$
\begin{aligned}
& \text { S.E.Jirm }=\sqrt{\frac{a c}{(a+c)^{3}}+\frac{b d}{(b+d)^{3}}}=\sqrt{\frac{(22)(2)}{(24)^{3}}+\frac{(12)(7)}{(19)^{3}}} \\
&= \sqrt{\frac{44}{13824}+\frac{84}{6859}}=\sqrt{0,003+0,012} \\
&=\sqrt{0,015}=0,122
\end{aligned}
$$


Para obter-se os intervalos de confiança de $95 \%$ para cada teste, o erro padrão é acrescentado ou diminuído do índice. Dessa forma:

$$
\begin{aligned}
& I C 95_{\text {Jveeg }}=0,268 \pm 0,137 \\
& I C 95_{\text {Jirm }}=0,285 \pm 0,122
\end{aligned}
$$

Em seguida, pode-se calcular o erro padrão da diferença (S.E.dif):

$$
\begin{aligned}
\text { S.E.dif } & =\sqrt{\left(S . E \cdot{ }_{1}\right)^{2}+(S . E \cdot 2)^{2}}=\sqrt{(0,137)^{2}+(0,122)^{2}} \\
& =\sqrt{0,018+0,014}=\sqrt{0,032}=0,178
\end{aligned}
$$

Finalmente, aplica-se o teste $\mathrm{t}$ aos valores:

$$
t=\frac{\text { diferença entre índices }}{\text { erro padrão da diferença }}=\frac{0,285-0,268}{0,178}=\frac{0,017}{0,178}=0,095
$$

Desta maneira, pode-se notar que, da mesma forma que a AUCROC, a diferença entre os índices de Youden não foi significativamente diferente entre os dois testes, uma vez que o resultado do teste t é bem inferior a 2,018. Consequentemente, não se pode excluir a hipótese nula, considerando-se portanto que os dois exames não possuem diferença estatisticamente significante de acurácia (Tabela 28).

Tabela 28: Índices de Youden.

\begin{tabular}{lccc}
\hline \hline & \multicolumn{2}{c}{ IC 95\% } \\
\hline \multirow{2}{*}{ VEEG } & Valor & Limite inf. & Limite sup. \\
IRM & 0,285 & 0,131 & 0,405 \\
\hline \hline
\end{tabular}





\section{Discussão}

\subsection{Interpretação dos resultados}

Em estudos de acurácia diagnóstica, os resultados de um ou mais testes sob análise são comparados com os desfechos determinados pelo padrão-ouro, sendo ambos aplicados numa população que compartilha uma condição de interesse. (BOSSUYT et al., 2015). No presente trabalho, esta condição de interesse é a epilepsia farmacorresistente em pacientes com displasia cortical focal sendo considerados para cirurgia. A localização da zona epileptogênica, que corresponde à região que deve ser operada para tornar um paciente livre de suas crises epilépticas, é obtida por meio da reunião clínica multidisciplinar. Desta forma, esta reunião gera uma hipótese de localização da zona epileptogênica. O objetivo, em toda cirurgia, é uma vida livre de crises epilépticas.

A complementação da hipótese de localização da zona epileptogênica com o desfecho pós-operatório nos casos operados é uma estratégia recomendada (BURCH et al., 2012). Sendo assim, o padrão-ouro deste estudo é a localização da região que foi operada, em um grupo de pacientes que tiveram mais de 12 meses de acompanhamento pós-operatório.

Este estudo possui relevância para a prática clínica pois coloca, em números, uma questão comum a todos os neurologistas envolvidos no cuidado de pacientes com epilepsia: o quanto a IRM e a VEEG apresentam convergência de resultados quando comparados com o local onde será realizada a cirurgia de epilepsia, numa população frequentemente atendida nos ambulatórios.

Entretanto, é necessário cuidado ao utilizar os resultados da análise da acurácia. A diferença não-significante entre os resultados, por si só, não pode ser considerada como uma inferência de que a VEEG e a IRM possuem a mesma acurácia diagnóstica. Para que isso acontecesse, seria necessário que o estudo tivesse poder estatístico suficiente para que houvesse segurança na afirmação de que os dois testes possuem a mesma acurácia. A ausência da evidência de diferença não necessariamente implica em evidência da existência de igualdade (ALTMAN; BLAND, 1995).

A epilepsia decorrente da DCF comumente se inicia nos primeiros anos de vida, inclusive podendo ocorrer logo após o parto (BAST et al., 2006). No presente estudo, a demografia da população estudada se aproxima da descrita na literatura, com predomínio de pacientes jovens, abaixo dos 28 anos de idade (FAUSER et al., 2006; FAUSER et al. 2015). A idade mediana à cirurgia foi de 15,5 anos, com duração mediana de 10,5 anos de epilepsia. Estes números são menores do que os de algumas séries, em que os pacientes operados são mais velhos (idade mediana de 19 anos) e com mais tempo de doença (tempo 
mediano de doença de 12 anos à cirurgia). (FAUSER et al., 2015, COHEN-GADOL et al., 2004).

Em 91,1\% dos pacientes em que foi realizada análise anatomopatológica da peça ressecada, foi confirmada a presença de DCF. Tumores, glioses e resultados indeterminados ocorreram em 8,9\% das amostras. Este valor é coerente com as descrições na literatura sobre correlação de achados na IRM com o resultado anatomopatológico (BAST et al., 2006, BRONEN et al., 1996: CAKIRER et al., 2002). A taxa de correlação de uma suposta DCF na IRM com sua confirmação histopatológica varia de 63 a $98 \%$ dos casos. A motivação para escolha de pacientes para análise baseados em sua descrição radiológica foi aproximar o estudo do neurologista atuante, que não dispõe dos resultados anatomopatológicos para exercer sua tomada de decisão.

Quanto à distribuição dos locais de cirurgia, os resultados estão de acordo com as séries descritas (KRAL et al., 2003, HAMIWKA et al., 2005), que indicam os lobos frontal e temporal como os mais comumente abordados. No presente estudo, os lobos frontal e temporal foram abordados em 44,7 e $25 \%$ dos casos, respectivamente. Cirurgias hemisféricas foram conduzidas em 10,8\% dos casos, um índice consistente com a literatura de séries mistas envolvendo adultos e crianças, que varia entre 2 e $16 \%$ (HARVEY et al. 2008, TISI et al. 2011).

O índice de monitorização invasiva, que foi conduzida em $73,2 \%$ dos pacientes, acompanha os índices de monitorizações invasivas realizadas em pacientes extrahipocampais, que podem variar de 41 a $85 \%$ dos casos (BERG et al., 2003; BAUTISTA et al., 2003). Em sua maioria, foram realizadas eletrocorticografias intraoperatórias, que ainda que não estejam conclusivamente associadas a algum valor prognóstico (CHERN et al., 2010, SPENCER; HUH, 2008), são úteis para a delimitação da área a ser ressecada (PALMINI et al., 1994, TRIPATHI et al., 2010).

O desfecho encontrado de $55 \%$ dos pacientes livres de crises epilépticas após um ano de cirurgia está dentro dos índices de sucesso de outros centros, cuja taxa de sucesso varia entre 52 e $67 \%$ (FAUSER et al., 2015, HADER et al., 2004). Esta taxa de sucesso é estável ao longo do tempo, com cerca de $80 \%$ dos pacientes mantendo sua condição livre de crises mesmo após 10 anos de cirurgia (FAUSER et al., 2015). Além disso, o benefício parece alcançar de forma semelhante tanto crianças quanto adultos (CHERN et al., 2010).

A especificidade e a sensibilidade são medidas fundamentais da acurácia diagnóstica (GLANTZ, 2005). Estes indicadores dependem das características da população estudada, portanto o uso de uma amostra pareada, ou seja, com cada sujeito recebendo os dois testes em análise, refina a comparação. A amostra foi homogênea para gênero e idade, com população notadamente jovem, e pequeno intervalo para a realização da cirurgia. Todos os sujeitos possuíam a mesma doença. 
Aproximou-se da realidade clínica ao se utilizar apenas laudos dos exames para análise. Não se procurou informações anotadas em prontuários, que poderiam ter sido modificadas pela reunião clínica, para diminuir a possibilidade de vieses, uma vez que, frequentemente, a avaliação de um exame muda à luz do resultado de outro. Tal mudança não necessariamente possui relevância clínica; de qualquer forma, buscou-se a forma mais pura possível de interpretação dos exames.

Os resultados indeterminados foram desconsiderados na avaliação. Isto também se aproxima da realidade clínica: ao se deparar com um resultado de IRM com lesão difusa ou de VEEG com zona de início ictal difusa, frequentemente se utiliza um terceiro método para avaliação da zona epileptogênica (como SPECT ictal) (VELASCO et al., 2002). De nota, cinco pacientes de 43 foram excluídos da análise em função da VEEG difusa.

Quanto à reprodutibilidade, o estudo é destinado ao neurologista em atividade no ambulatório de epilepsia. A intenção é que o profissional conheça o quanto dois exames frequentemente utilizados na prática clínica apresentam convergência de resultados, numa população de pacientes específica.

Alguns estudos previamente avaliaram acurácia diagnóstica em cirurgia de epilepsia. Novos métodos de investigação foram validados, como a localização de fontes eletroencefalográficas (BENICZKY et al., 2013), e instrumentos convencionalmente realizados na avaliação pré-cirúrgica de epilepsia foram questionados, como o SPECT (tomografia por emissão de fóton único) na epilepsia do lobo temporal com descargas interictais bilaterais (VELASCO et al. 2002) e o VEEG prolongado em crianças com lesões unilaterais (PATIL et al. 2008) ou epilepsia do lobo temporal associada à esclerose hipocampal (CENDES et al. 2000). Nestes três estudos, redundância nos métodos foi observada, e a revisão do seu uso foi sugerida. Achados discordantes entre IRM e VEEG foram encontrados para gangliogliomas, e a cirurgia no local da lesão à IRM apresentou bons resultados (MORRIS et al. 1998). Cirurgias também já foram conduzidas com sucesso em pacientes com padrões ictais generalizados ao VEEG e que apresentavam lesões epileptogênicas focais (WYLLIE et al., 2007).

Por outro lado, poupar a lesão epileptogênica e conduzir cirurgia em uma região distante, guiada pela localização das zonas de início ictais identificadas à VEEG também foi reportada, com bons resultados (HOLMES et al., 1999). Nesta casuística, $50 \%$ dos pacientes ficaram livres de crises. Entretanto, esta conduta cirúrgica foi aplicada em apenas $5 \%$ dos casos operados.

\subsection{Limitações}

Devido à restrição de tempo, não foi possível a análise inicial de 764 pacientes, tendo sido avaliados 209 pacientes $(27,35 \%)$ e utilizados 58 paciente na análise de convergência e 


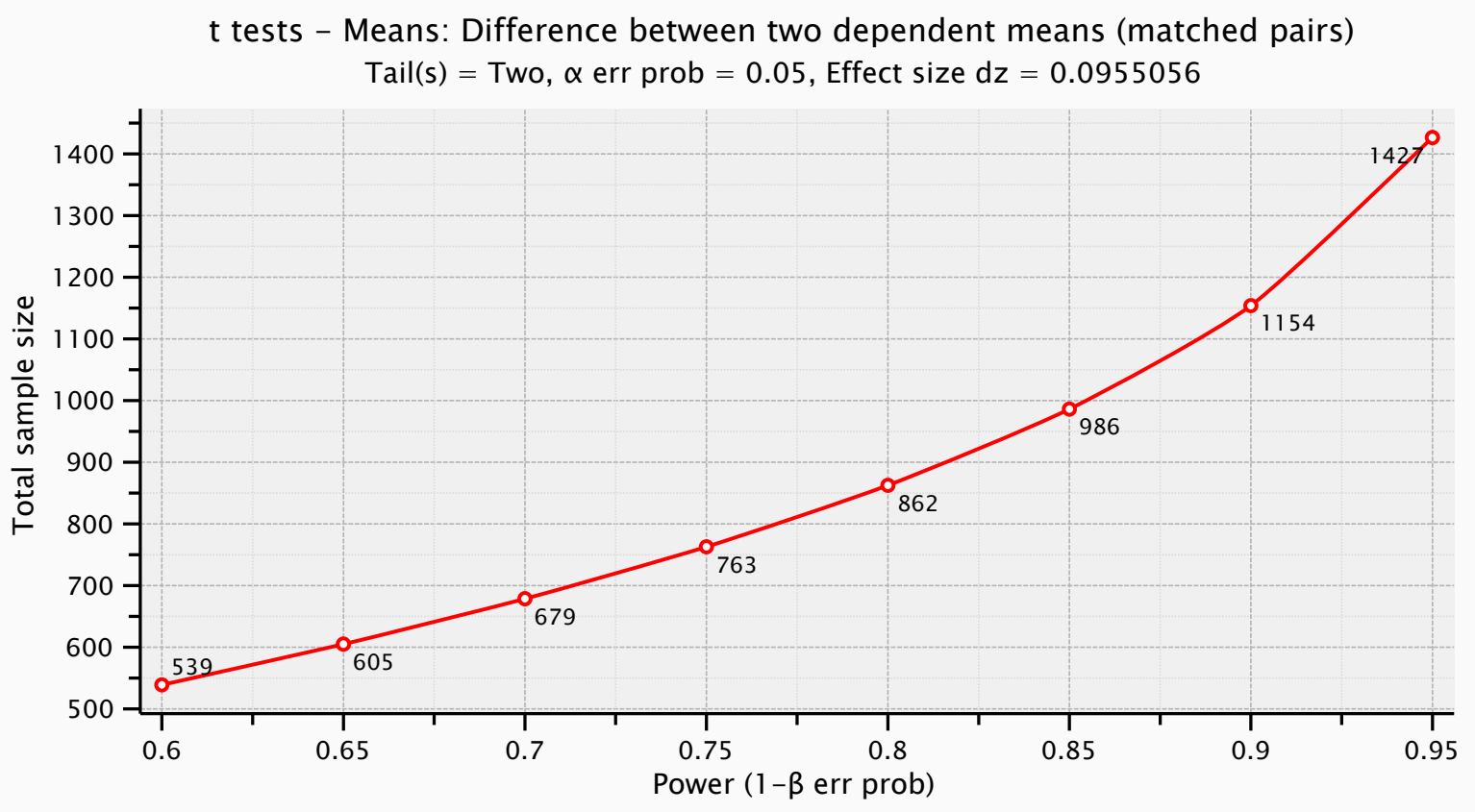

Figura 8: Cálculo do poder estatístico do estudo.

43 pacientes na análise de acurácia. Utilizando G*Power 3.1.9.2 (FAUL et al., 2007), e baseado nos resultados do índice de Youden, considerando uma diferença de 0,017 entre os valores e erro padrão da diferença de 0,178, o poder estatístico do estudo foi de 9,3\% (Figura 8). Para um poder do teste convencional de 80\%, seriam necessários 862 pacientes.

O baixo poder estatístico do estudo pode ser creditado tanto à pequena amostra quando à estreita diferença no desempenho dos testes. Seu significado é que a chance de descobrir efeitos genuinamente positivos é baixa, ou seja, tendem a produzir mais resultados falso-negativos (BUTTON et al., 2013). Além disso, o presente estudo não é capaz de indicar se os dois exames em avaliação possuem uma acurácia diagnóstica semelhante. Pode existir uma pequena diferença clinicamente relevante, que este estudo não foi capaz de identificar (erro tipo II) (BERRY; COUSTĖRE-YAKIR; GROVER, 1998).

\subsection{Futuro}

Pesquisas subsequentes no campo do estudo da comparação da acurácia diagnóstica entre VEEG e IRM poderiam utilizar diferentes etiologias, como esclerose hipocampal ou tumor. Além disso, poderiam utilizar os mesmos critérios, numa população maior, para obter resultados por subgrupos. Isto refletiria melhor a realidade dos pacientes que estão envolvidos no espectro da epilepsia focal farmacorresistente.

Investigações no campo da acurácia diagnóstica da videoeletroencefalografia prolongada e da ressonância magnética de encéfalo podem aproveitar os dados do presente estudo para planejamento do tamanho da amostra, minimizando a probabilidade de ocorrência 
de erros tipo II (BERRY; COUSTĖRE-YAKIR; GROVER, 1998).

\subsection{Aplicação}

Finalmente, pode-se instruir o neurologista que, confrontado com um paciente com epilepsia focal farmacorresistente lesional associada à displasia cortical focal e candidato à lesionectomia, a videoeletroencefalografia prolongada e a imagem de ressonância magnética de encéfalo possuem uma moderada convergência de resultados. Além disso, não possuem diferença estatisticamente significante quanto à acurácia diagnóstica na determinação da zona epileptogênica. Porém, não se pode afirmar que os dois exames possuem a mesma acurácia. 

Parte III

\section{Conclusão}





\section{Conclusão}

\subsection{Conclusão principal}

Os resultados da videoeletroencefalografia prolongada e da ressonância magnética de encéfalo apresentaram um nível moderado de concordância.

\subsection{Conclusão secundária}

A imagem de ressonância magnética de encéfalo e a videoeletroencefalografia prolongada não possuem diferença estatisticamente significante quanto à acurácia para determinação da zona epileptogênica, região a ser ressecada nos pacientes com displasia cortical focal sendo avaliados para cirurgia de epilepsia. 



\section{Referências}

ALTMAN, D. G.; BLAND, J. M. Statistics notes: Absence of evidence is not evidence of absence. $B M J$, v. 311, n. 7003, p. 485-485, 1995. Citado na página 73.

AMERICAN ELECTROENCEPHALOGRAPHIC SOCIETY. Guideline Twelve: Guidelines for Long-Term Monitoring for Epilepsy. Journal of Clinical Neurophysiology, Hagerstown, v. 25, n. 3, p. 170-180, 2008. Citado na página 34.

ANDERMANN, F. Herbert Henri Jasper 1906-1999. Epilepsia, Malden, v. 41, n. 1, p. 113-120, 2000. Citado na página 35.

BAST, T. et al. Focal cortical dysplasia: Prevalence, clinical presentation and epilepsy in children and adults. Acta Neurologica Scandinavica, Copenhagen, v. 113, n. 2, p. 72-81, 2006. Citado 2 vezes nas páginas 73 e 74 .

BAUTISTA, J. F. et al. Focal cortical dysplasia and intractable epilepsy in adults: Clinical, EEG, imaging, and surgical features. Epilepsy Research, Amsterdã, v. 55, n. 1-2, p. 131-136, 2003. Citado na página 74.

BENICZKY, S. et al. Source localization of rhythmic ictal EEG activity: A study of diagnostic accuracy following STARD criteria. Epilepsia, Malden, v. 54, n. 10, p. 1743-1752, 2013. Citado 4 vezes nas páginas 31, 47, 48 e 75.

BERG, A. T. et al. Revised terminology and concepts for organization of seizures and epilepsies: Report of the ILAE Commission on Classification and Terminology, 2005-2009. Epilepsia, Malden, v. 51, n. 4, p. 676-685, 2010. Citado 2 vezes nas páginas 27 e 36.

BERG, A. T. et al. The Multicenter Study of Epilepsy Surgery: Recruitment and Selection for Surgery. Epilepsia, Malden, v. 44, n. 11, p. 1425-1433, 2003. Citado na página 74.

BERGER, H. Das Elektrenkephalogramm des Menschen. Die Naturwissenschaften, Berlim, v. 23, n. 8, p. 121-124, 1935. Citado na página 33.

BERRY, E. M.; COUSTÈRE-YAKIR, C.; GROVER, N. B. The significance of non-significance. Quaterly Journal of Medicine, Oxford, v. 91, n. 9, p. 647-653, 1998. Citado 2 vezes nas páginas 76 e 77.

BIGGERSTAFF, B. J. Comparing diagnostic tests: A simple graphic using likelihood ratios. Statistics in Medicine, Chichester, v. 19, n. 5, p. 649-663, 2000. Citado na página 48 .

BLOCH, F. Nuclear Induction. Physical Review, Lancaster, v. 70, p. 460, 1946. Citado na página 32 .

BLUMCKE, I. et al. The clinicopathologic spectrum of focal cortical dysplasias: A consensus classification proposed by an ad hoc Task Force of the ILAE Diagnostic Methods Commission. Epilepsia, Malden, v. 52, n. 1, p. 158-174, 2011. Citado na página 28. 
BOESCH, C. Nobel Prizes for nuclear magnetic resonance: 2003 and historical perspectives. Journal of Magnetic Resonance Imaging, Chicago, v. 20, n. 2, p. 177-179, 2004. Citado na página 31 .

BORGES, M. A. et al. Urban prevalence of epilepsy: Populational study in São José do Rio Preto, a medium-sized city in Brazil. Arquivos de Neuro-Psiquiatria, São Paulo, v. 62, n. 2 A, p. 199-205, 2004. Citado na página 26.

BOSSUYT, P. M. et al. STARD 2015: An updated list of essential items for reporting diagnostic accuracy studies. Clinical Chemistry, Washington, v. 61, n. 12, p. 1446-1452, 2015. Citado 3 vezes nas páginas 31, 43 e 73 .

BRONEN, R. et al. Refractory epilepsy: Comparison of MR imaging, CT, and histopathologic findings in 117 patients. Radiology, Easton, v. 201, n. 1, p. 97-105, 1996. Citado na página 74 .

BURCH, J. et al. Dilemmas in the interpretation of diagnostic accuracy studies on presurgical workup for epilepsy surgery. Epilepsia, Malden, v. 53, n. 8, p. 1294-1302, 2012. Citado 6 vezes nas páginas 31, 37, 43, 47, 59 e 73.

BURNEO, J. G.; TELLEZ-ZENTENO, J.; WIEBE, S. Understanding the burden of epilepsy in Latin America: A systematic review of its prevalence and incidence. Epilepsy Research, Amsterdã, v. 66, n. 1-3, p. 63-74, 2005. Citado na página 26.

BUTTON, K. S. et al. Power failure: why small sample size undermines the reliability of neuroscience. Nature reviews. Neuroscience, Nature Publishing Group, Londres, v. 14, n. 5, p. 365-76, 2013. Citado na página 76.

CAKIRER, S. et al. MR imaging in epilepsy that is refractory to medical therapy. European Radiology, Berlim, v. 12, n. 3, p. 549-558, 2002. Citado na página 74.

CARPIO, A. et al. Mortality of epilepsy in developing countries. Epilepsia, Malden, v. 46, n. SUPPL. 11, p. 28-32, 2005. Citado na página 26.

CATARINO, C. B.; VOLLMAR, C.; NOACHTAR, S. Paradoxical lateralization of non-invasive electroencephalographic ictal patterns in extra-temporal epilepsies. Epilepsy Research, Amsterdã, v. 99, n. 1-2, p. 147-155, 2012. Citado na página 36.

CENDES, F. et al. Is Ictal Recording Mandatory in Temporal Lobe Epilepsy? Archives of Neurology, Chicago, v. 57, n. 4, p. 497, 2000. Citado na página 75.

CHEN, H. H. et al. Cognitive and epilepsy outcomes after epilepsy surgery caused by focal cortical dysplasia in children: early intervention maybe better. Child's Nervous System, Berlim, v. 30, n. 11, p. 1885-1895, 2014. Citado na página 29.

CHERN, J. J. et al. Surgical outcome for focal cortical dysplasia: an analysis of recent surgical series. Journal of neurosurgery. Pediatrics, Charlottesville, v. 6, n. 5, p. 452-458, 2010. Citado na página 74 .

COHEN-GADOL, A. a. et al. Long-term outcome after epilepsy surgery for focal cortical dysplasia. Journal of Neurosurgery, Charlottesville, v. 101, p. 55-65, 2004. Citado na página 74. 
COMISSION ON CLASSIFICATION AND TERMINOLOGY OF THE INTERNATIONAL LEAGUE AGAINST EPILEPSY. Proposal for revised clinical and electroencephalographic classification of epileptic seizures. Epilepsia, Malden, v. 22, n. 4, p. 489-501, 1981. Citado na página 27.

COMISSION ON CLASSIFICATION AND TERMINOLOGY OF THE INTERNATIONAL LEAGUE AGAINST EPILEPSY. Proposal for Revised Classification of Epilepsies and Epileptic Syndromes. Epilepsia, Malden, v. 30, n. 4, p. 389-399, 1989. Citado na página 27.

CRINO, P. B. Focal Cortical Dysplasia. Semin Neurol, Nova Iorque, v. 35, n. 3, p. 201-208, 2015. Citado 2 vezes nas páginas 28 e 29.

DASH, G. K. et al. An audit of the presurgical evaluation and patient selection for extratemporal resective epilepsy surgery in a resource-poor country. Seizure, BEA Trading Ltd, Londres, v. 21, n. 5, p. 361-366, 2012. Citado na página 37.

DOBESBERGER, J. et al. Video-EEG monitoring: Safety and adverse events in 507 consecutive patients. Epilepsia, Malden, v. 52, n. 3, p. 443-452, 2011. Citado na página 35.

ENGEL, J. Report of the ILAE classification core group. Epilepsia, Malden, v. 47, n. 9, p. 1558-1568, 2006. Citado na página 27.

FAUL, F. et al. G* Power 3 : A flexible statistical power analysis program for the social , behavioral, and biomedical sciences. Behavior Research Methods, Austin, v. 39, n. 2, p. 175-191, 2007. Citado na página 76.

FAUSER, S. et al. Long-term seizure outcome in 211 patients with focal cortical dysplasia. Epilepsia, Malden, v. 56, n. 1, p. 66-76, 2015. Citado 2 vezes nas páginas 73 e 74.

FAUSER, S. et al. Clinical characteristics in focal cortical dysplasia: A retrospective evaluation in a series of 120 patients. Brain, Oxford, v. 129, n. 7, p. 1907-1916, 2006. Citado 2 vezes nas páginas 29 e 73.

FISHER, R. S. et al. ILAE Official Report: A practical clinical definition of epilepsy. Epilepsia, Malden, v. 55, n. 4, p. 475-482, 2014. Citado 2 vezes nas páginas 25 e 26.

FISHER, R. S. et al. Epileptic seizures and epilepsy: Definitions proposed by the International League Against Epilepsy (ILAE) and the International Bureau for Epilepsy (IBE). Epilepsia, Malden, v. 46, n. 4, p. 470-472, 2005. Citado na página 25.

FOLDVARY, N. et al. The localizing value of ictal EEG in focal epilepsy. Neurology, Hagerstown, v. 57, n. 11, p. 2022-2028, 2001. Citado na página 36.

FOUNTAIN, N. B. et al. Quality improvement in neurology: AAN epilepsy quality measures: Report of the quality measurement and reporting subcommittee of the American Academy of Neurology. Neurology, Hagerstown, v. 76, n. 1, p. 94-99, 2011. Citado na página 26 .

GLANTZ, S. A. The special case of two groups: the t test. In: GLANTZ, S. A. (Ed.). Primer of Biostatistics. 6. ed. Nova Iorque: Hill, Mc-Graw, 2005. cap. 4, p. 73-123.

Citado 3 vezes nas páginas 68, 69 e 74 . 
HADER, W. J. et al. Cortical dysplastic lesions in children with intractable epilepsy: role of complete resection. Journal of neurosurgery, Charlottesville, v. 100, n. 2 Suppl Pediatrics, p. 110-117, 2004. Citado na página 74.

HAMIWKA, L. et al. Surgery for epilepsy due to cortical malformations: Ten-year follow-up. Epilepsia, Malden, v. 46, n. 4, p. 556-560, 2005. Citado na página 74.

HANLEY, J. A.; MCNEIL, B. J. A method of comparing the areas under receiver operating characteristic curves derived from the same cases. Radiology, Easton, v. 148, n. 3, p. 839-43, 1983. Citado na página 69.

HARVEY, A. S. et al. Defining the spectrum of international practice in pediatric epilepsy surgery patients. Epilepsia, Malden, v. 49, n. 1, p. 146-155, 2008. Citado na página 74.

HAUPTMAN, J. S.; MATHERN, G. W. Surgical treatment of epilepsy associated with cortical dysplasia: 2012 update. Epilepsia, Malden, v. 53, n. Supl. 4, p. 98-104, 2012.

Citado na página 26.

HAWASS, N. E. D. Comparing the sensitivities and specificities of two diagnostic procedures performed on the same group of patients. British Journal of Radiology, Londres, v. 70, n. 832, p. 360-366, 1997. Citado 3 vezes nas páginas 64, 65 e 66.

HELMSTAEDTER, C. Neuropsychological aspects of epilepsy surgery. Epilepsy and Behavior, São Diego, v. 5, n. Supl. 1, 2004. Citado na página 36.

HOLMES, M. D. et al. Hippocampal or Neocortical Lesions on Magnetic Resonance Imaging Do Not Necessarily Indicate Site of Ictal Onsets in Partial Epilepsy. Annals of Neurology, Boston, v. 45, n. 4, p. 461-465, 1999. Citado na página 75.

HORSLEY, V. Remarks on ten consecutive cases of operations upon the brain and cranial cavity to illustrate the details and safety of the method deployed. British Medical Journal, Londres, v. 1, n. 1373, p. 863-865, 1887. Citado na página 35.

IVANOVA, J. I. et al. Direct and indirect costs associated with epileptic partial onset seizures among the privately insured in the United States. Epilepsia, Malden, v. 51, n. 5, p. 838-844, 2010. Citado na página 26.

IVES, J. R.; THOMPSON, C. J.; GLOOR, P. Seizure monitoring: A new tool in electroencephalography. Electroencephalography and Clinical Neurophysiology, Amsterdã, v. 41, n. 4, p. 422-427, 1976 . Citado na página 33.

JACKSON, G. D.; KUZNIECKY, R. I.; PELL, G. S. Principles of magnetic resonance imaging. In: KUZNIECKY, R. I.; JACKSON, G. D. (Ed.). Magnetic Resonance in Epilepsy. Londres: Elsevier B.V., 2005. p. 22. Citado na página 32.

KAMP, A. Eight-channel EEG telemetering. Electroencephalography and Clinical Neurophysiology, Amsterdã, v. 15, n. 1, p. 164-166, 1963. Citado na página 33.

KLEM, G. et al. The ten-twenty electrode system of the International Federation.

Electroencephalography and Clinical Neurophysiology, Amsterdã, v. 52, n. Supl. 52, p. 3-6, 1999. Citado na página 45.

KRAL, T. et al. Outcome of epilepsy surgery in focal cortical dysplasia. J Neurol Neurosurg Psychiatry, Londres, v. 74, n. 2, p. 183-188, 2003. Citado na página 74. 
KUMAR, A.; CHUGANI, H. T. The Role of Radionuclide Imaging in Epilepsy, Part 1: Sporadic Temporal and Extratemporal Lobe Epilepsy. Journal of Nuclear Medicine, Reston, v. 54, n. 10, p. 1775-1782, 2016. Citado na página 36.

KUTSY, R. L.; FARRELL, D. F.; OJEMANN, G. A. Ictal Patterns of Neocortical Seizures Monitored with Intracranial Electrodes : Correlation with Surgical Outcome. Epilepsia, Malden, v. 40, n. 3, p. 257-266, 1994. Citado na página 36.

KWAN, P. et al. Definition of drug resistant epilepsy : Consensus proposal by the ad hoc Task Force of the ILAE Commission on Therapeutic Strategies. Epilepsia, Malden, v. 51, n. 6, p. 1069-1077, 2010. Citado na página 26.

KWAN, P.; BRODIE, M. J. Early identification of refractory epilepsy. New England Journal of Medicine, Boston, v. 342, n. 5, p. 314-319, 2000. Citado na página 26.

LANDIS, J.; KOCH, G. The measurement of observer agreemente for categorical data. Biometrics, Alexandria, v. 33, n. 1, p. 159-174, 1977. Citado na página 47.

LAUTERBUR, P. C. Image Formation by Induced Local Interactions: Examples Employing Nuclear Magnetic Resonance. Nature, Londres, v. 242, n. 5394, p. 190-191, 1973. Citado na página 31.

LEY, M. et al. Safety study of long-term video-electroencephalogram monitoring. Neurología (English Edition), SEGO, Barcelona, v. 29, n. 1, p. 21-26, 2014. Citado na página 34.

LI, L. M. et al. Demonstration project on epilepsy in Brazil: situation assessment. Arquivos de Neuro-Psiquiatria, São Paulo, v. 65, n. Supl 1, p. 5-13, 2007. Citado na página 26.

LI, L. M. et al. High resolution magnetic resonance imaging in adults with partial or secondary generalised epilepsy attending a tertiary referral unit. Journal of Neurology, Neurosurgery, and Psychiatry, Londres, v. 59, n. 4, p. 384-387, 1995. Citado na página 29 .

LIU, J.; MENG, F.; LIU, Z. Seizure-related adverse events during video- electroencephalography. Epileptic Disorders, Montrouge, v. 14, n. 5, p. 51-56, 2012. Citado na página 34.

LODDENKEMPER, T.; KOTAGAL, P. Lateralizing signs during seizures in focal epilepsy. Epilepsy and Behavior, São Diego, v. 7, n. 1, p. 1-17, 2005. Citado na página 36.

MANFORD, M.; FISH, D. R.; SHORVON, S. D. An analysis of clinical seizure patterns and their localizing value in frontal and temporal lobe epilepsies. Brain, Londres, v. 119, n. 1, p. 17-40, 1996. Citado na página 36.

MANSFIELD, P.; MAUDSLEY, A. A. Medical imaging by NMR. British Journal of Radiology, Londres, v. 50, n. 591, p. 188-194, 1977. Citado na página 32.

MORRIS, H. H. et al. Ganglioglioma and Intractable Epilepsy : Clinical and Neurophysiologic Features and Predictors of Outcome After Surgery. Epilepsia, Malden, v. 39, n. 3, p. 307-313, 1998. Citado na página 75. 
MUHLEBNER, A. et al. Neuropathologic measurements in focal cortical dysplasias : validation of the ILAE 2011 classification system and diagnostic implications for MRI. Acta Neuropathologica, Berlim, v. 123, n. 2, p. 259-272, 2012. Citado na página 29.

OBUCHOWSKI, N. A.; LIEBER, M. L.; WIANS, F. H. ROC Curves in Clinical Chemistry : Uses, Misuses, and Possible Solutions. Clinical Chemistry, Washington, v. 50, n. 7, p. 1118-1125, 2004. Citado na página 67 .

PALMINI, A. et al. Operative strategies for patients with cortical dysplastic lesions and intractable epilepsy. Epilepsia, Malden, v. 35 Suppl 6, p. S57-S71, 1994. Citado na página 74.

PATIL, S. G. et al. Is streamlined evaluation of children for epilepsy surgery possible ? Epilepsia, Malden, v. 49, n. 8, p. 1340-1347, 2008. Citado na página 75.

RAMSEY, N. F. Early History of Magnetic Resonance. Bulletin of Magnetic Resonance, Filadélfia, v. 1, n. 2, p. 94-99, 1999. Citado na página 31.

RIZVI, S. A. A. et al. Is rapid withdrawal of anti-epileptic drug therapy during video EEG monitoring safe and efficacious ? Epilepsy Research, Elsevier B.V., Amsterdã, v. 108, n. 4, p. 755-764, 2014. Citado na página 34.

ROSENOW, F.; LUDERS, H. Presurgical evaluation of epilepsy. Brain, Londres, v. 124, n. 9, p. 1683-1700, 2001. Citado 3 vezes nas páginas 34, 35 e 36.

SCHAAFSMA, J. D. et al. Decision analysis to complete diagnostic research by closing the gap between test characteristics and cost-effectiveness. Journal of Clinical Epidemiology, Elsevier Inc, Nova Iorque, v. 62, n. 12, p. 1248-1252, 2009. Citado 2 vezes nas páginas 30 e 31.

SCHIJNS, O. E. M. G. et al. The start and development of epilepsy surgery in Europe : a historical review. Neurosurgical Review, Berlim, v. 38, n. 3, p. 447-461, 2015. Citado na página 35.

SCHUELE, S. U.; LUDERS, H. O. Intractable epilepsy : management and therapeutic alternatives. Lancet Neurology, Londres, v. 7, n. 6, p. 514-524, 2008. Citado na página 35.

SCOTT, R. A.; LHATOO, S. D.; SANDER, J. W. A. S. The treatment of epilepsy in developing countries : where do we go from here ? Bulletin of the World Health Organization, Londres, v. 79, n. 4, p. 344-351, 2001. Citado na página 26.

SIGTAP. Sistema de gerenciamento da tabela de procedimentos, medicamentos e OPM do SUS. 2015. Disponível em: <www.sigtap.datasus.gov.br>. Citado 2 vezes nas páginas 33 e 34 .

SO, E. L. Value and Limitations of Seizure Semiology in Localizing Seizure Onset. Journal of Clinical Neurophysiology, Nova Iorque, v. 23, n. 4, p. 353-357, 2006. Citado na página 36.

SPENCER, S.; HUH, L. Outcomes of epilepsy surgery in adults and children. The Lancet Neurology, Londres, v. 7, n. 6, p. 525-537, 2008. Citado na página 74. 
STONE, J. et al. Who is referred to neurology clinics ? - The diagnoses made in 3781 new patients. Clinical Neurology and Neurosurgery, Elsevier B.V., Amsterdã, v. 112, n. 9, p. 747-751, 2010. Citado na página 26.

TAUSSIG, D.; MONTAVONT, A.; ISNARD, J. Invasive EEG explorations Explorations EEG invasives. Neurophysiologie Clinique / Clinical Neurophysiology, Elsevier Masson SAS, Paris, v. 45, n. 1, p. 113-119, 2015. Citado na página 36.

TAYLOR, D. C. et al. Focal dysplasia of the cerebral cortex in epilepsy. Journal of Neurology, Neurosurgery, and Psychiatry, Londres, v. 34, n. 4, p. 369-387, 1971. Citado na página 28.

TAYLOR, D. C.; MARSH, S. M. Hughlings Jackson' s Dr Z : the paradigm of temporal lobe epilepsy revealed. Journal of Neurology, Neurosurgery, and Psychiatry, Londres, v. 43, n. 9, p. 758-767, 1980. Citado na página 35.

TISI, J. D. et al. The long-term outcome of adult epilepsy surgery, patterns of seizure remission, and relapse: A cohort study. The Lancet, Londres, v. 378, n. 9800, p. 1388-1395, 2011. Citado na página 74.

TONG, X. et al. Validation of the Generalized Anxiety Disorder-7 ( GAD-7 ) among Chinese people with epilepsy. Epilepsy Research, Elsevier B.V., Amsterdã, v. 120, p. 31-36, 2016. Citado na página 48.

TRIPATHI, M. et al. Intra-operative electrocorticography in lesional epilepsy. Epilepsy Research, Amsterdã, v. 89, n. 1, p. 133-141, 2010. Citado na página 74.

URBACH, H. Imaging of the epilepsies. European Radiology, Berlim, v. 15, n. 3, p. 494-500, 2005. Citado na página 36.

VASSARSTATS. VassarStats: Website for Statistical Computation. 2015. Disponível em: $<$ www.vassarstats.net $>$. Citado na página 47.

VELASCO, T. R. et al. Accuracy of ictal SPECT in mesial temporal lobe epilepsy with bilateral interictal spikes. Neurology, Hagerstown, v. 59, n. 2, p. 266-271, 2002. Citado na página 75 .

VELASCO, T. R.; MATHERN, G. W. Surgical treatmente of refractory temporal lobe epilepsy. In: WYLLIE, E. (Ed.). Wyllie's Treatment of Epilepsy. 5. ed. Filadélfia: Lippincott Williams and Wilkins, 2011. cap. 82, p. 908-922. Citado na página 26.

VELEZ-RUIZ, N. J.; KLEIN, J. P. Neuroimaging in the Evaluation of Epilepsy. Seminars in Neurology, Nova Iorque, v. 32, n. 4, p. 361-373, 2012. Citado na página 27.

WANG, Z. I. et al. The pathology of magnetic-resonance- imaging-negative epilepsy. Modern Pathology, Nature Publishing Group, Baltimore, v. 26, n. 8, p. 1051-1058, 2013. Citado na página 29.

WELLMER, J. et al. Proposal for a magnetic resonance imaging protocol for the detection of epileptogenic lesions at early outpatient stages. Epilepsia, Malden, v. 54, n. 11, p. 1977-1987, 2013. Citado na página 33.

WESTMORELAND, B. F. The EEG Findings in Extratemporal Seizures. Epilepsia, Malden, v. 39, n. Supplement 4, p. 1-8, 1998. Citado na página 35. 
WIESER, H. G. et al. Proposal for a New Classification of Outcome with Respect to Epileptic Seizures Following Epilepsy Surgery. Epilepsia, Malden, v. 42, n. 2, p. 282-286, 2001. Citado na página 44

WYLLIE, E. et al. Successful surgery for epilepsy due to early brain lesions despite generalized EEG findings. Neurology, Hagerstown, v. 69, n. 4, p. 389-397, 2007. Citado na página 75 .

YOUDEN, W. J. Index for rating diagnostic tests. Cancer, Hoboken, v. 3, n. 1, p. 32-35, 1950. Citado 3 vezes nas páginas 48,69 e 70.

ZWEIG, H.; CAMPBELL, G. Receiver-Operating Clinical Medicine ( ROC ) Plots : A Fundamental Evaluation Tool in. Clinical Chemistry, Washington, v. 39, n. 4, p. 561-577, 1993. Citado 2 vezes nas páginas 67] 68. 
Apêndices 

APÊNDICE A - Índice para localização de dados (iniciativa STARD). 


\begin{tabular}{lccc}
\hline \hline & Número do item & Página & Parágrafo \\
\hline Título e resumo & 1 & - & - \\
Introdução & 2 & 25 & 1 \\
Participantes & 3 & 43 & 1 \\
Recrutamento & 4 & 44 & 1 \\
Seleção & 5 & 43 & 1 \\
Coleta de dados & 6 & 44 & 2 \\
Padrão de referência & 7 & 43 & 1 \\
Especificações técnicas & 8 & 45 & 1 \\
Categorias de resultados & 9 & 44 & 2 \\
Responsáveis pelos resultados & 10 & 44 & 2 \\
Mascaramento & 11 & 45 & 3 \\
Métodos estatísticos & 12 & 47 & 1 \\
Reprodutibilidade & 13 & 73 & 3 \\
Data de recrutamento & 14 & 44 & 1 \\
Demografia & 15 & 51 & 6 \\
Critérios de inclusão (diagrama) & 16 & 52 & - \\
Intervalo entre testes e padrão & 17 & 53 & - \\
Critérios para condição-alvo & 18 & 44 & 4 \\
Tabelas cruzadas & 19 & 64 & - \\
Eventos adversos & 20 & - & - \\
Estimativas de acurácia & 21 & 63 & - \\
& & 64 & - \\
Variação da acurácia entre subgrupos & 23 & 65 & - \\
Estimativa de reprodutibilidade & 24 & 68 & - \\
Discussão da aplicação clínica & 25 & 71 & - \\
\hline \hline
\end{tabular}


APÊNDICE B - Artigo submetido ao periódico Seizure 
Elsevier Editorial System(tm) for Seizure:

European Journal of Epilepsy

\author{
Manuscript Draft
}

Manuscript Number: 2016-141

Title: Accuracy of LTM and MRI in focal cortical dysplasia epilepsy surgery.

Article Type: Full Length Article

Keywords: epilepsy; cortical dysplasia; long-term video-EEG monitoring; magnetic resonance imaging; epilepsy surgery; accuracy.

Corresponding Author: Mr. Bruno Zanotelli Monnerat, M.D.

Corresponding Author's Institution: Hospital das Clinicas de Ribeirao Preto

First Author: Bruno Zanotelli Monnerat, M.D.

order of Authors: Bruno Zanotelli Monnerat, M.D.; Americo C Sakamoto, PhD Manuscript Region of Origin: BRAZIL

Abstract: Purpose: The aim of the study was to investigate the accuracy of long-term videoelectroencephalography monitoring (LTM) and brain magnetic resonance imaging (MRI) in patients with focal cortical dysplasia in the work-up of epilepsy surgery.

Methods: Medical records of 209 patients were reviewed, and 43 were considered in the analysis. All had LTM and MRI studies. The region the surgeon operated upon, based on the conclusions of the multidisciplinary meeting, in a group of seizure-free patients was considered the reference standard. The regions of seizure-onset zone (LTM) results and the epileptogenic lesion (MRI) results were compared between each other and with the reference standard. Global indexes of accuracy were performed and compared.

Results: The sensibility and specificity of LTM (86.3\% [CI 95\% 64-96.4] and 36.8\%, [CI 95\% 17.2-61.3] respectively) were not statistically different from those of MRI (95.8\% [CI 95\% 76.8-99.7] and 31.5\% [CI 95\% 13-56]). In addition, Youden's indexes and areas under the Roc curves were not significantly different, meaning that the diagnostic accuracy of LTM and MRI are equivalent in this clinical scenario. A substantial level of concordance $(\mathrm{k}=0.654)$ was observed between LTM and MRI.

Conclusion: LTM and MRI have equivalent accuracy for epilepsy surgery work-up in a selected group of patients. Surgery might proceed without LTM in this group, contributing to a streamlined process and releasing monitoring beds for diagnostic purposes.

Suggested Reviewers: Sandor Beniczky

sbzdiladelfia.dk

Previous work on accuracy in epilepsy surgery. 
Jane Burch

jane.burcheyork.ac.uk

Previous work on accuracy in epilepsy.

Kurupath Radhakrishnan

kurupath.radhakrishnandgmail. com

Previous work on epilepsy surgery in resource-poor countries. 


\section{Cover Letter}

Ribeirão Preto, Brazil, March 29, 2016

Dear Editor,

I am writing this letter as an introduction to the research article I have just submitted for publication in Seizure, entitled 'Accuracy of MRI and LTM in focal cortical dysplasia epilepsy surgery'. This is from the material of my doctoral thesis, conducted here in Ribeirão Preto, Brazil, under the supervision of Prof Américo C Sakamoto. The approval from our ethics committee on research was properly obtained, and there are no financial interests or funding bodies supporting this study.

In our institution, all patients being considered for epilepsy surgery receive long-term videoelectroencephalography monitoring (LTM). When dealing with refractory extratemporal lobe epilepsy patients being considered for lesionectomy, I have always wondered whether LTM would convey the same information about the epileptogenic zone as the magnetic resonance imaging (MRI) of the brain does. The number of patients operated on isolated dysplastic lesions noted on MRI is overwhelming, regardless of the results of LTM. Intuitively, I had the perception that both tests (MRI and LTM) would convey the same information, and the surgeon needs an MRI for surgery. Therefore, LTM might be redundant in selected cases. Fortunately, our observations led us to conclude that this is indeed the case.

We designed a retrospective accuracy study in a population of patients with refractory extratemporal epilepsy associated with focal cortical dysplasia, comparing LTM and MRI. First, we analysed medical records looking for reports of both studies. After that, we selected patients based on whether surgery was performed or not. When performed, outcome regarding seizures after 12 postoperative months was registered. Then, we investigated whether LTM and MRI findings agreed, and also the regions the surgeon decided to operate upon based on the conclusions of a presurgical multidisciplinary meeting. We observed that the sensitivity and specificity of MRI and LTM for the determination of the epileptogenic region were not significantly different. Moreover, additional global measures of accuracy were performed and compared (areas under ROC curves and Youden's indexes), and again, no difference was found. Both tests had equivalent accuracy in this clinical scenario.

This study could be used as an indication that LTM might be waived in selected patients being considered for epilepsy surgery. This could streamline epilepsy surgery, especially in a resource-poor country such as Brazil. Further, we could release LTM beds for proper epilepsy diagnosis, in my mind, the most precise application of this instrument.

We have identified a limitation to presurgical LTM in a common population where it is used, contributing to the understanding of this method, and we believe that an audience of neurologists involved in the care of similar patients with epilepsy in long-term monitoring units would welcome such information. Additionally, I would like to declare that none of the authors has any conflicts of interest.

Thank you very much for your time,

Bruno Z Monnerat, Neurologist

Epilepsy Surgery Centre, Hospital das Clínicas de Ribeirão Preto, Brazil

bzmonnerat@usp.br 


\section{${ }^{*}$ Conflict of Interest}

Disclosure of conflicts of interest: None of the authors has any conflicts of interest to disclose. 


\begin{abstract}
Purpose: The aim of the study was to investigate the accuracy of long-term videoelectroencephalography monitoring (LTM) and brain magnetic resonance imaging (MRI) in patients with focal cortical dysplasia in the work-up of epilepsy surgery.

Methods: Medical records of 209 patients were reviewed, and 43 were considered in the analysis. All had LTM and MRI studies. The region the surgeon operated upon, based on the conclusions of the multidisciplinary meeting, in a group of seizure-free patients was considered the reference standard. The regions of seizure-onset zone (LTM) results and the epileptogenic lesion (MRI) results were compared between each other and with the reference standard. Global indexes of accuracy were performed and compared. Results: The sensibility and specificity of LTM (86.3\% [CI 95\% 64-96.4] and 36.8\%, [CI 95\% 17.2-61.3] respectively) were not statistically different from those of MRI (95.8\% [CI 95\% 76.8-99.7] and 31.5\% [CI 95\% 13-56]). In addition, Youden's indexes and areas under the ROC curves were not significantly different, meaning that the diagnostic accuracy of LTM and MRI are equivalent in this clinical scenario. A substantial level of concordance $(k=0.654)$ was observed between LTM and MRI. Conclusion: LTM and MRI have equivalent accuracy for epilepsy surgery work-up in a selected group of patients. Surgery might proceed without LTM in this group, contributing to a streamlined process and releasing monitoring beds for diagnostic purposes.
\end{abstract}


*Manuscript

Click here to view linked References

Accuracy of LTM and MRI in focal cortical dysplasia epilepsy surgery.

Bruno Z. Monnerat ${ }^{\mathrm{a}}$, Américo C. Sakamoto ${ }^{\mathrm{a}}$.

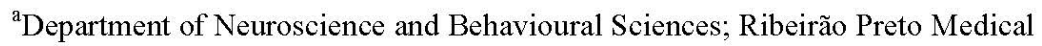

School, University of São Paulo, Ribeirão Preto, Brazil.

Corresponding author: Bruno Z Monnerat; Av. Bandeirantes, 3900, CEP 14049-900,

Ribeirão Preto/SP, Brazil. E-mail: bzmonnerat@usp.br; Phone +55 16 3602-2613; Fax

$+55163633-0760$.

Keywords: epilepsy, cortical dysplasia, long-term video-EEG monitoring, magnetic resonance imaging, epilepsy surgery, accuracy. 


\section{Introduction}

Surgery is an efficacious treatment for the common and serious condition that is epilepsy $(1,2)$. About $1-2 \%$ of the population has epilepsy, and in about $35 \%$ of them medications provide no control of seizures $(3,4)$. This medically refractory population is responsible for almost $80 \%$ of the monetary cost of epilepsy in the USA (5). The chances of a seizure-free life after epilepsy surgery vary from $38 \%$ to $66 \%$, whereas conservative treatment is effective in only about $16 \%(6)$. The risk of sudden unexplained death in epilepsy diminishes in patients successfully operated on (7), and quality of life significantly improves (8)

Unfortunately, access to epilepsy surgery is still inadequate in many places, whether because of unawareness of the population or physicians about the possibilities of this treatment method or because of the high costs involved and the intense resources employed in the diagnostic methods classically used for presurgical evaluation of epilepsy $(9 ; 10)$. This surgical treatment gap is astonishingly shared between both developing and developed nations, including the USA, where the mean time from epilepsy start to surgery is 22 years (10 years of that already in a refractory condition) (11).

LTM remains one of the standard tools for the definition of the seizure-onset zone needed for planning the epilepsy surgery work-up (12). It is also invaluable for proper management and diagnosis of various clinical conditions, usually enabling the differentiation between epileptic seizures and non-epileptic events, and also the identification and classification of distinct epilepsy syndromes and related conditions $(13,14)$. Additionally, LTM allows the distinction between true epileptogenic and incidental brain lesions which can even be of the same nature (for example, tubers, cavernomas, hamartomas, brain calcifications) and may provide useful information to 
assess the extension of the epileptogenic zone and the networks involved in the expression of seizures. However, LTM is associated with a plethora of risks and inconveniences, which, although rare, could may be prevented in a large proportion of patients $(15,16)$. Moreover, the costs and resources needed to start and maintain such facilities are considerable, and out of reach from a large part of the world (17).

In developing countries, more than $70 \%$ of the patients being referred for LTM are epilepsy surgery candidates (18), compared with $30 \%$ in developed countries (19). The pressure on epilepsy centres to conduct surgery in medically refractory patients is huge everywhere, but mostly in developing countries, where the paucity of resources and large pool of patients demand pragmatic solutions $(17,20,21,10)$. Moreover, proper use of LTM leads to an improvement in the diagnosis of epilepsy, and medical management changed in about $73 \%$ of patients admitted to an LTM facility (19).

MRI of the brain is the neuroimaging method of choice for epilepsy $(22,23)$. It is an indispensable diagnostic tool for both the neurologist and neurosurgeon involved in the care of epilepsy patients, being employed in the detection of the anatomical substrates of disease (epileptogenic lesions). Lesional epilepsies, where an epileptogenic lesion is found on MRI, have a more favourable postsurgical prognosis compared with non-lesional surgeries (24).

However, the objectives of the presurgical evaluation go far beyond the simply identification and localization of potentially epileptogenic lesions. It should provide a wide set of critical information necessary to define not only eligibility but also the best surgical strategy for any given patient. In extratemporal epilepsies, in particular, it is indispensable for adequate surgical planning to assess beforehand the extension and limits of the putative epileptogenic zone (not the same as epileptogenic lesion) and its spatial relationships, whenever the case, to highly functional cortical areas overlapping 
or bordering it, especially in lesions of known imprecise limits such as cortical dysplasias and gliosis.

Motivation for this study was the observation that, usually, the convergence of results from LTM and MRI regarding the localisation of the epileptogenic zone, that is, the region where surgery must be performed for a seizure-free status be obtained (12), is high. Therefore, the objective was to analyse and compare, in a group of refractory epilepsy patients with single, extra-hippocampal focal cortical dysplasia, the diagnostic accuracy of both LTM and MRI for the localisation of the epileptogenic zone. The hypothesis is that, if equivalency in accuracy is obtained, streamlined epilepsy surgery shortening LTM, could be offered.

\section{Methods}

\subsection{Study design and participants}

This is a retrospective observational study of accuracy that was conducted at the Epilepsy Surgery Centre of the Hospital das Clínicas de Ribeirão Preto, São Paulo, Brazil, between February 2013 and July 2014. The study was approved by the local research ethics committee.

Recruitment was from the Epilepsy Surgery Centre database, with searched-for patients fulfilling the following inclusion criteria: a) LTM report indicating the localisation of the seizure-onset zone; b) MRI report indicating the localisation of the extrahippocampal epileptogenic lesion; c) MRI report mentioning probable focal cortical dysplasia (FCD); d) epilepsy surgery conducted in the Epilepsy Surgery Centre; and e) first admission for LTM. All patients received both LTM and MRI, and were sampled consecutively. 
We excluded patients satisfying any of the following criteria: a) more than one epileptogenic lesion; b) diffuse or subcortical epileptogenic lesion; c) absence of epileptogenic lesion on MRI report; d) absence of LTM report; e) no seizures on LTM; e) previous epilepsy surgery; or f) evaluation during status epilepticus.

\subsection{Test methods}

Medical charts were reviewed by the principal investigator (BZM). Both LTM and MRI reports preceded the conclusions of the multidisciplinary meeting that leads to epilepsy surgery in our Centre. The 2 index tests were the localisation of the epileptogenic region in the MRI report and the localisation of the seizure-onset zone in the LTM report.

The reference standard was the region of the performed surgery (lobar level) supplemented by the outcome following surgery, as recommended in the literature (25). This region of surgery was obtained from the weekly multidisciplinary meeting consensus before epilepsy surgery for all patients. In this meeting, experienced epileptologists, neuroradiologists, neuropsychologists, psychiatrists and neurosurgeons evaluated LTM, MRI and other presurgical testes for the consensus decision to proceed or not to surgery, and defined its localisation and surgical strategies if appropriate (26).

The protocol for data acquisition of both LTM and MRI is described elsewhere (27). Reports from the index tests and conclusions of the multidisciplinary meeting (region of surgery) were signed by attending physicians; the index tests were assessed without knowledge of the site at which surgery was to be conducted. Outcome information was derived from clinical notes made by supervised neurology residents 
during outpatient postsurgical visits, and was not blinded to the results of the tests' evaluations. Neuroimaging is frequently performed previous to admission, so MRI images and reports could be reviewed at LTM. The only information derived from the multidisciplinary meeting was the region of surgery; all other information predated the meeting, except for surgical follow-up status. The localisation of the seizure-onset zone (LTM) and of the epileptogenic lesion (MRI) was annotated regarding cerebral lobe and hemisphere. Diffuse MRI cases referred to bilateral epileptogenic lesions; diffuse LTM cases referred to non-lateralised seizure-onsets.

Concordant patients were the ones in which the localisation of the epileptogenic lesion (MRI) or the seizure-onset zone (LTM) results concurred with the region of surgery. When the MRI or LTM differed from the region of surgery, the case was considered discordant.

The last available postsurgical seizure outcome was used, according to Engel's classification (28), separating seizure-free (Engel I) and not seizure-free (Engel II, III, IV) patients. All had at least 1 year of postsurgical follow-up; $48.8 \%$ of the sample had up to 5 years of follow-up, and $51.2 \%$ had more than 5 years of follow-up.

\subsubsection{Analysis}

The intended review of 764 patients was not possible due to time constraints; 209 consecutive patients were reviewed ( $27.35 \%$ of intended sample size), and 43 were deemed eligible for the study (participants).

Statistical analysis was performed using SPSS Statistics 22 (IBM Corp., Armonk, USA). Sensitivity was calculated as the percentage of true-positive patients (i.e., patients whose localisation of the seizure-onset zone or the epileptogenic lesion 
was concordant with the region of surgery, and seizure-free status was obtained) divided by the total of seizure-free patients (these being concordant or discordant). Specificity was calculated according to the formula: number of true negatives/(number of true negatives + number of false positives). True negatives were considered when there was discordance between the seizure-onset zone or the epileptogenic lesion and the region of surgery, and the patient was not seizure-free. False positives were characterised as patients in whom surgery was conducted in a region of concordance but in whom seizure-free status was not obtained. False negatives were patients in whom surgery was conducted in a region of discordance and from whom seizure-free status was obtained $(25)$.

Predictive values were calculated also based on the outcome status. Positive predictive values were calculated as the number of true positives/(number of true positives + number of false positives). The negative predictive values were calculated as the number of true negatives/(number of true negatives + number of false negatives).

To assess the clinical value of each test, likelihood ratios were also considered. Positive likelihood ratios were sensitivity/(1-specificity), and negative likelihood ratios were (1-sensitivity)/specificity (29).

Finally, two global measures of accuracy were estimated: Youden's index (30) and the areas under the receiver-operating characteristic curves (AUROC) (31). Youden's index is calculated by deducting 1 from the sum of the test's sensitivity and specificity, as in: (sensitivity + specificity) -1 . The AUROC is an estimation of the discriminative power of a test, calculated based on a plot considering sensitivity and specificity. Both global measures result in values varying between zero and 1 , with better accuracy associated with higher values. 
When comparing two diagnostic tests, to verify for equivalence or even the superiority of one over the other, every measure of accuracy (such as sensitivity and specificity) generates a value; its confidence interval must be ascertained for precision, and the difference between the 2 values must be subjected to statistical analysis to check for significance.

To evaluate the differences in sensibilities and specificities between LTM and MRI, McNemar's test was used (32). The difference between Youden's indexes was calculated using a paired $t$-test (30). The difference between the two AUROCs was analysed using Pearson's correlation test (31). To analyse the convergence between LTM and MRI, Cohen's kappa was used. Kappa values were considered poor $(k<0)$, slight (0.01-0.2), fair $(0.21-0.4)$, moderate $(0.41-0.6)$, substantial $(0.61-0.8)$ and almost perfect $(>0.8)(33)$.

\section{Results}

Patient selection followed the diagram presented in Figure 1. Eligible patients (participants) were tested using both MRI and LTM, according to the flowchart in Figure 2. Demographic and clinical information of the participants is summarised in Table 1 . 

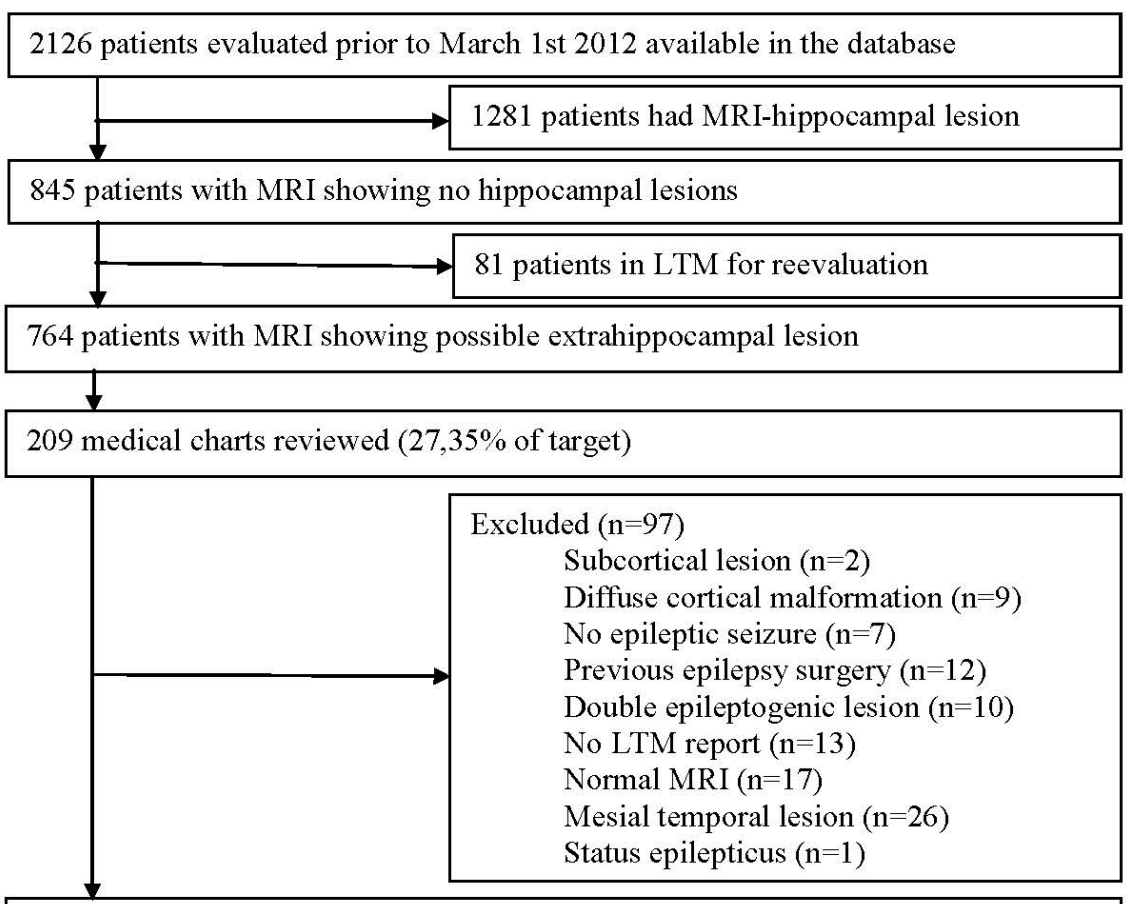

112 patients included

$\longrightarrow$\begin{tabular}{c|} 
No focal cortical dysplasia $(\mathrm{n}=54)$ \\
Gliosis $(\mathrm{n}=25)$ \\
Tumour $(\mathrm{n}=17)$ \\
Vascular malformation $(\mathrm{n}=6)$ \\
Undetermined lesion $(\mathrm{n}=2)$ \\
Other lesions $(\mathrm{n}=4)$
\end{tabular}

58 patients with focal cortical dysplasia selected

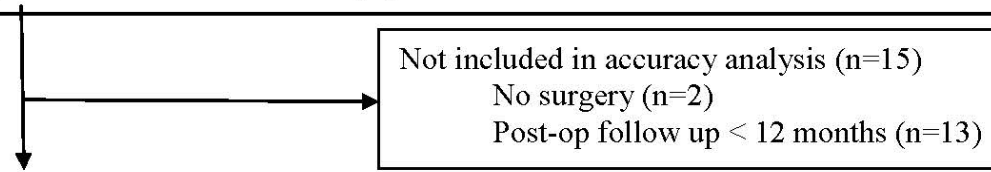

43 patients were eligible for accuracy analysis.

Figure 1. Patient selection. LTM: long-term video-EEG monitoring. MRI: magnetic resonance imaging of the brain. 


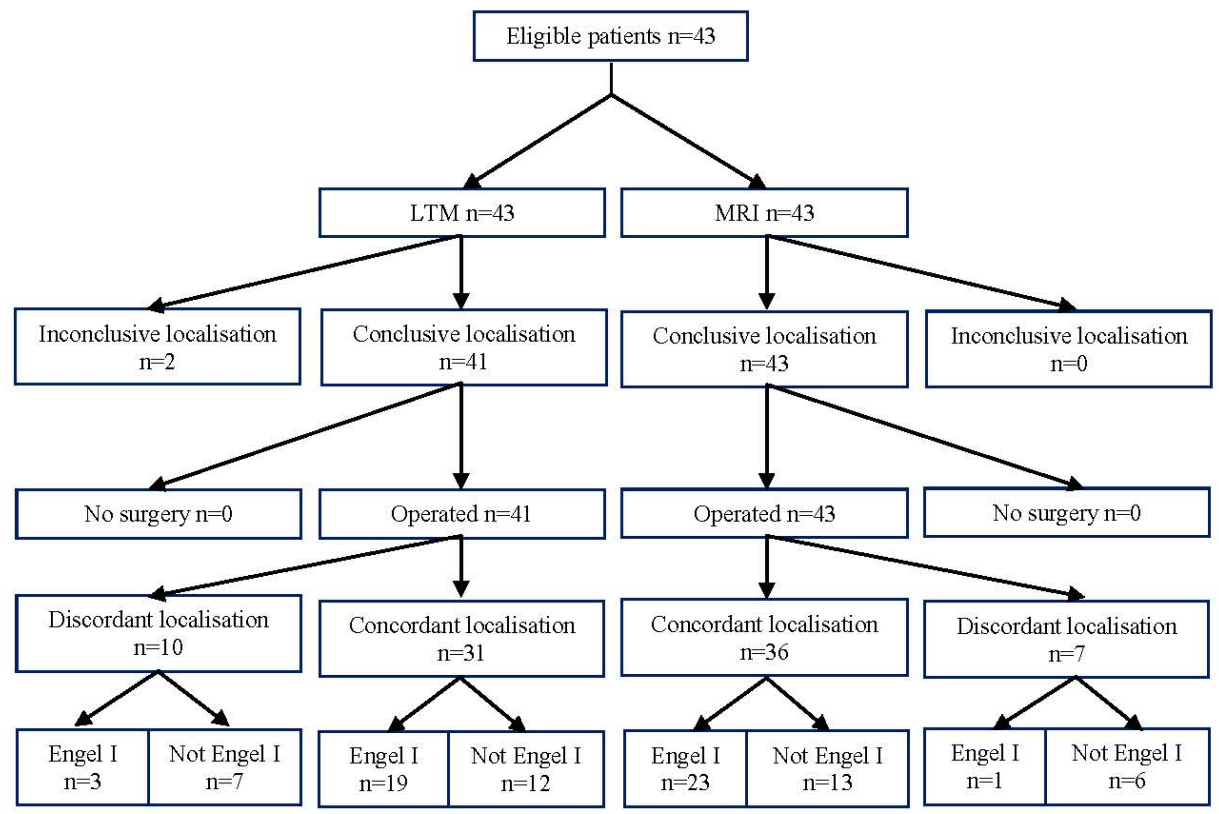

Figure 2. Flowchart of results. LTM: long-term video-EEG monitoring. MRI: magnetic resonance imaging of the brain. Reference standard: region of surgery (at lobar level) in seizure-free patients. Concordant localisation: match between the index test and the reference standard. Discordant localisation: no match between the index test and the reference standard.

\subsection{Participants}

The study analysed 43 patients, being $25(58.1 \%)$ females and $18(41.9 \%)$ males, with age at surgery ranging from 2 to 58 years (mean 18.7 years). Mean epilepsy duration was 13.44 years at surgery. The interval between LTM and surgery was less than 12 months in $76.8 \%$ of patients (mean 9.33 months). 
Upon inspection of MRI reports, FCD was found in the left hemisphere in $51.2 \%$ of the patients and on the right in $48.8 \%$ of the patients. The most frequent localisations of lesions were frontal (37.2\%), temporal (32.6\%), parieto-occipital (14\%), hemispheric (9.3\%) and central (7\%). Most patients had 1.5 T MRI (74.4\%).

Regarding LTM findings, seizure-onset zones were detected in the left hemisphere in $47.7 \%$ patients and in the right hemisphere in $47.7 \%$ of the patients. In the remaining $4.6 \%$ of the patients, the seizure-onset zone was non-lateralised. The most frequent localisations of seizure-onset zones were frontal (39.5\%), temporal (23.7\%), hemispheric (15.8\%). parieto-occipital (13.2\%) and central (7.9\%).

Invasive studies were performed in $80 \%$ of the patients, in the majority of the cases these procedures were only intraoperatively ( $48.8 \%$ of the patients). Few patients $(11.6 \%)$ required chronic invasive evaluations and cortical stimulation because due to proximity to eloquent regions. At last outpatient visit, $55.8 \%$ of the patients were seizure-free.

\begin{tabular}{lc}
\hline \multicolumn{2}{l}{ Table 1. Demographic and clinical characteristics of the 43 eligible patients. } \\
\hline \multicolumn{2}{l}{ Female; N (\%) } \\
\hline Mean age at LTM; years (range) & $25(58.1)$ \\
\hline Mean epilepsy duration at LTM; years (range) & $17.17(1-58)$ \\
\hline Mean age at surgery; years (range) & $11.79(0-49)$ \\
\hline Mean epilepsy duration at surgery; years (range) & $18.70(2-58)$ \\
\hline Mean follow-up post-surgery; years (range) & $13.44(1-49)$ \\
\hline 1.5T MRI; N (\%) & $6.4(1-16)$ \\
\hline Availability of routine EEG; N (\%) & $32(74.4)$ \\
\hline Invasive studies performed; N (\%) & $14(32.6)$ \\
Acute only & $21(48.8)$ \\
Chronic & $11(25.6)$ \\
\hline Cortical stimulation performed; N (\%) \\
Acute only \\
Chronic & $8(18.6)$ \\
\hline Seizure-free at last appointment; N (\%) & $5(11.6)$ \\
\hline
\end{tabular}




\subsection{Test results}

The target condition was seizure-free status at least 12 months after surgery. Both index tests provided localisation data that were compared with localisation of surgery. In case of convergence between localisation of the index test and localisation of surgery, concordance was assumed. If the results were different, discordance ensued. Regarding MRI data, of the 43 patients analysed thirty-six were concordant with site of surgery. In 23 of them, seizure-free status was obtained. In 7 patients, discordant results were found, and only one patient was found to be seizure-free.

The data from LTM originated from 41 patients because 2 patients were excluded due to inconclusive localisation (34). In 31 patients, there was concordance with the region of surgery, and 19 of these patients were seizure-free at last follow-up. In 10 patients, discordance was observed, and 3 patients were seizure-free.

Two cross-tables were constructed from the data gathered (Table 2), and analysis of diagnostic accuracy can proceed (Table 3). The difference in sensitivity was 9.5\% favouring MRI, and in specificity 5.3\% favouring LTM. However, both results did not meet statistical significance. The difference in the AUROCs was of 0.057 favouring MRI (Figure 3), and Youden's index was better in MRI by 0.042 points; however, neither was statistically significant.

The kappa value for the agreement between LTM and MRI was 0.654 (substantial). 
Table 2. Cross tabulation of the index tests results and the reference standard

a) LTM

\begin{tabular}{cccc}
\hline & Seizure-free & Not seizure-free & \\
\hline Concordant & $19(\mathrm{TP})$ & $12(\mathrm{FP})$ & 31 \\
\hline Discordant & $3(\mathrm{FN})$ & $7(\mathrm{TN})$ & 10 \\
\hline & 22 & 19 & 41 \\
\hline
\end{tabular}

b) MRI

\begin{tabular}{cccc}
\hline & Seizure-free & Not seizure-free & \\
\hline Concordant & $23(\mathrm{TP})$ & $13(\mathrm{FP})$ & 36 \\
\hline Discordant & $1(\mathrm{FN})$ & $6(\mathrm{TN})$ & 7 \\
\hline & 24 & 19 & 43 \\
\hline
\end{tabular}

$\mathrm{n}=$ patients; TP: true positives; FN: false negatives; FP: false positives; TN: true negatives

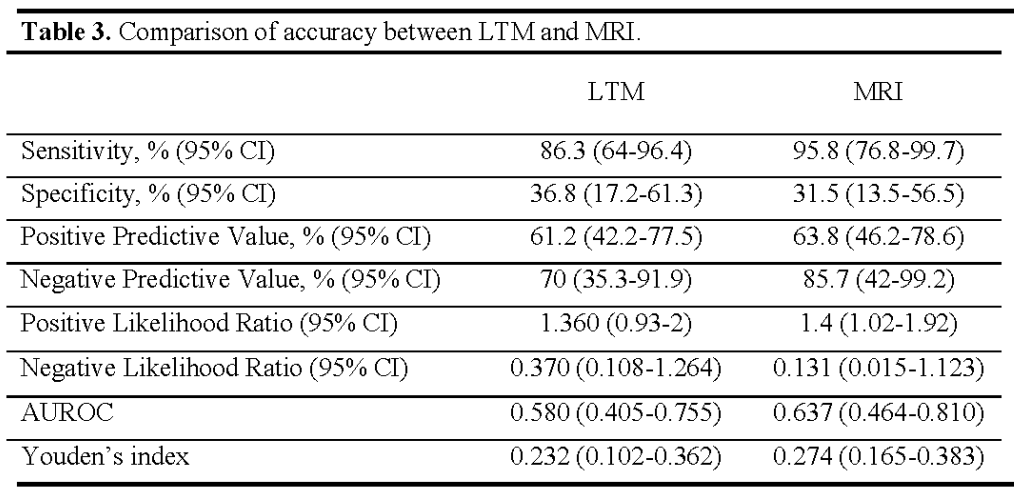

All differences non-significant $(P>0.05)$. 


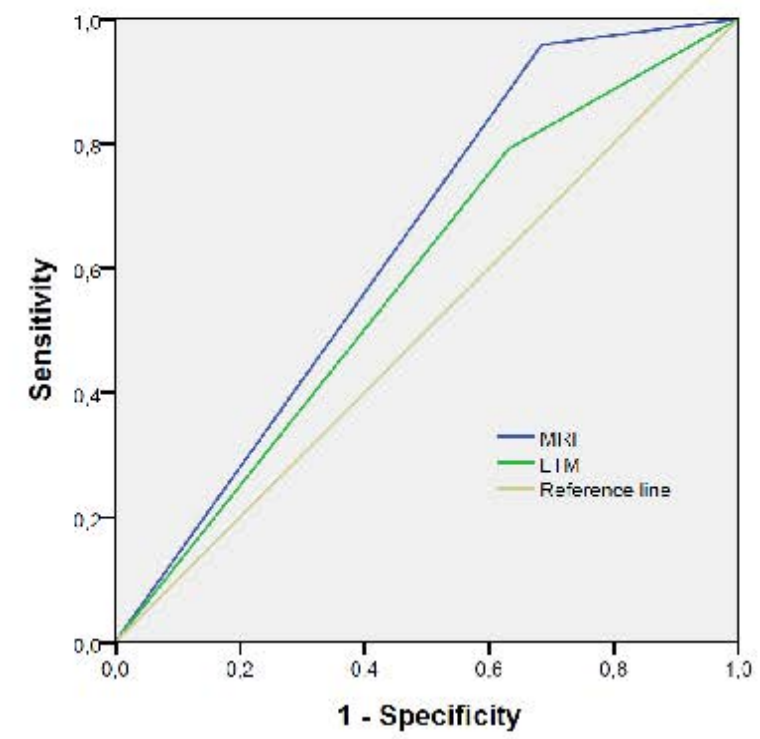

ligure 3. Receiver-operating characterislic (ROC) curves, L LM: lonth-1erm vdeo-T.E. monitoring. MRI: magnetic resonance imaging of the brain.

\section{Discussion}

In a group of 43 patients with focal cortical dysplasia and medically refractory epilepsy who were surgically treated, LTM and MRI reports were compared with the epileptogenic zone, and equivalence in diagnostic accuracy was found between them. The limitations of this study are the restricted criteria for inclusion and the small number of participants. However, the sample is homogeneous and the aetiology selected is common in epilepsy centres. Moreover, the study followed recommended STARD (Standards for Reporting of Diagnostic Accuracy Studies) criteria (34).

When a te st has both a higher sensitivity and higher specificity, it is clearly preferred. However, when one test has a higher sensitivity and the other a higher specificity, which test to prefer is not al ways clear. Two summary indexes can be 
employed in this situation: the AUROC and Youden's index (35). The use of Youden's index is a novelty in epilepsy surgery accuracy studies. This index represents the difference between the true-positive rate and the false-negative rate, and is an appropriate tool for comparing accuracies because of its clarity and simplicity (35). Its value is directly related to the AUROC, the conventional way of comparing diagnostic test, and it represents the ideal cut-off score in continuous data studies (36).

Many studies have previously evaluated diagnostic accuracy in epilepsy surgery. New methods of investigation have been validated, such as EEG source localisation (26), and investigations usually performed in the standard work-up of epilepsy surgery have been questioned, such as ictal single-photon emission computed tomography for hippocampal sclerosis temporal lobe epilepsy with bilateral discharges (37), and LTM for epilepsy surgery in children with unilateral lesions (38) or hippocampal sclerosis temporal lobe epilepsy (39). In these three studies, redundancy of the methods was observed, and reviewing of its use was advised. Discordant LTM and MRI findings were reported for gangliogliomas (40), and operating on the lesion had good results. Surgery has been successfully conducted in patients with generalised EEG ictal patterns and focal, early epileptogenic lesions (41).

Sparing the epileptogenic lesion and conducting surgery in a distant region, guided by seizure-onset zones, has been reported (42) with good results (50\% seizurefree). However, it represented less than $5 \%$ of the Centre's epilepsy surgeries. In the present study, only one patient was operated on distant from the epileptogenic lesion, and seizure-free status was not obtained.

The chances of a seizure-free outcome are poor in the absence of a wellcircumscribed lesion on MRI (43). This is crucial information to reduce the treatment gap currently faced by many low-income countries. Simplifying access to epilepsy 
surgery could reduce this gap (10) and promote the differential diagnosis of epilepsy

and other seizure disorders by potentially increasing availability of LTM beds.

\section{Conclusion}

The diagnostic accuracy of MRI and LTM is equivalent for the gross localization of the epileptogenic zone for epilepsy surgery work-up in a sample of extrahippocampal focal cortical dysplasia patients. This suggests that LTM could be shortened and used more objectively in the presurgical evaluation from the work-up in selected cases, streamlining epilepsy surgery work-up.

Acknowledgements: This study was accomplished with the invaluable assistance of Drs Tonicarlo R Velasco, Ana Paula P. Martins, Veriano Alexandre Jr., and Frederico N Nakano and Mrs Elidia Magnani, Adriana Nogueira and Silvana Lo Turco. 


\section{References}

1. Wiebe S, Blume WT, Girvin JP, Eliasziw M. Effectiveness and Efficiency of Surgery for Temporal Lobe Epilepsy Study Group. A randomized, controlled trial of surgery for temporal-lobe epilepsy. N Engl J Med. 2001 Aug 2;345(5):311-8.

2. Engel J Jr, Wiebe S, French J, Sperling M, Williamson P, Spencer D, Gumnit R, Zahn C, Westbrook E, Enos B. Practice parameter: temporal lobe and localized neocortical resections for epilepsy. Epilepsia. 2003 Jun;44(6):741-51.

3. Borges MA, Min LL, Guerreiro CA, Yacubian EM, Cordeiro JA, Tognola WA, Borges AP, Zanetta DM. Urban prevalence of epilepsy: populational study in São José do Rio Preto, a medium-sized city in Brazil. Arq Neuropsiquiatr. 2004 Jun;62(2A):199204.

4. Kwan P, Brodie MJ. Early identification of refractory epilepsy. N Engl J Med. 2000 Feb 3;342(5):314-9

5. Begley CE, Famulari M, Annegers JF, Lairson DR, Reynolds TF, Coan S, Dubinsky S, Newmark ME, Leibson C, So EL, Rocca WA. The cost of epilepsy in the United States: an estimate from population-based clinical and survey data. Epilepsia. 2000 Mar;41(3):342-51. 
6. Bien CG, Szinay M, Wagner J, Clusmann H, Becker AJ, Urbach H. Characteristics and surgical outcomes of patients with refractory magnetic resonance imaging-negative epilepsies. Arch Neurol. 2009 Dec;66(12):1491-9. doi:10.1001/archneurol.2009.283.

7. Terra VC, Scorza FA, Cavalheiro EA, Wichert-Ana L, Pinto KG, Machado HR,Sakamoto AC. Pediatric epilepsy surgery and sudden unexpected death epilepsy: the contribution of a Brazilian epilepsy surgery program. Childs Nerv Syst. 2010 Aug;26(8):1075-9. doi: 10.1007/s00381-010-1108-z.

8. Spencer SS, Berg AT, Vickrey BG, Sperling MR, Bazil CW, Haut S, Langfitt JT, Walczak TS, Devinsky O; Multicenter Study of Epilepsy Surgery. Health-related quality of life over time since resective epilepsy surgery. Ann Neurol. 2007 Oct; $62(4): 327-34$.

9. Mbuba CK, Ngugi AK, Newton CR, Carter JA. The epilepsy treatment gap in developing countries: a systematic review of the magnitude, causes, and intervention strategies. Epilepsia. 2008 Sep;49(9):1491-503. doi:10.1111/j.1528-1167.2008.01693.x.

10. Radhakrishnan K. Challenges in the management of epilepsy in resource-poor countries. Nat Rev Neurol. 2009 Jun;5(6):323-30. doi: 10.1038/nrneurol.2009.53.

11. Berg AT, Vickrey BG, Testa FM, Levy SR, Shinnar S, DiMario F, Smith S. How long does it take for epilepsy to become intractable? A prospective investigation. Ann Neurol. 2006 Jul;60(1):73-9. 
12. Rosenow F, Lüders H. Presurgical evaluation of epilepsy. Brain. 2001 Sep;124(Pt 9):1683-700.

13. Nordli DR Jr. Usefulness of video-EEG monitoring. Epilepsia. 2006;47 Suppl 1:2630.

14. Alving J, Beniczky S. Diagnostic usefulness and duration of the inpatient long-term video-EEG monitoring: findings in patients extensively investigated before the monitoring. Seizure. 2009 Sep;18(7):470-3. doi:10.1016/j.seizure.2009.04.005.

15. Arrington DK, Ng YT, Troester MM, Kerrigan JF, Chapman KE. Utility and safety of prolonged video-EEG monitoring in a tertiary pediatric epilepsy monitoring unit. Epilepsy Behav. 2013 May;27(2):346-50. doi:10.1016/j.yebeh.2013.02.027.

16. Noe KH, Drazkowski JF. Safety of long-term video-electroencephalographic monitoring for evaluation of epilepsy. Mayo Clin Proc. 2009 Jun;84(6):495-500. doi: 10.1016/S0025-6196(11)60580-6.

17. Rathore C, Rao MB, Radhakrishnan K. National epilepsy surgery program: realistic goals and pragmatic solutions. Neurol India. 2014 Mar-Apr;62(2):124-9. doi: $10.4103 / 0028-3886.132318$.

18. Chemmanam T, Radhakrishnan A, Sarma SP, Radhakrishnan K. A prospective study on the cost-effective utilization of long-term inpatient video-EEG monitoring in a developing country. J Clin Neurophysiol. 2009 Apr;26(2):123-8. doi: 
10.1097/WNP.0b013e31819d8030.

19. Ghougassian DF, d'Souza W, Cook MJ, O'Brien TJ. Evaluating the utility of inpatient video-EEG monitoring. Epilepsia. 2004 Aug;45(8):928-32.

20. Asadi-Pooya AA, Sperling MR. Strategies for surgical treatment of epilepsies in developing countries. Epilepsia. 2008 Mar;49(3):381-5.

21. Wu XT, Li L, Yan B, Stefan H, Lei D, Zhou D. How to effectively constrain the cost of presurgical evaluation for resective surgery in low-income population: clinically oriented opinions. Seizure. 2011 Jun;20(5):425-7. doi:10.1016/j.seizure.2011.01.013.

22. Urbach H. Imaging of the epilepsies. Eur Radiol. 2005 Mar;15(3):494-500.

23. Wellmer J, Quesada CM, Rothe L, Elger CE, Bien CG, Urbach H. Proposal for a magnetic resonance imaging protocol for the detection of epileptogenic lesions at early outpatient stages. Epilepsia. 2013 Nov;54(11):1977-87. doi:10.1111/epi.12375.

24. Téllez-Zenteno JF, Hernández Ronquillo L, Moien-Afshari F, Wiebe S. Surgical outcomes in lesional and non-lesional epilepsy: a systematic review and meta-analysis. Epilepsy Res. 2010 May;89(2-3):310-8. doi:10.1016/j.eplepsyres.2010.02.007.

25. Burch J, Marson A, Beyer F, Soares M, Hinde S, Wieshmann U, Woolacott N.

Dilemmas in the interpretation of diagnostic accuracy studies on presurgical workup for 
epilepsy surgery. Epilepsia. 2012 Aug;53(8):1294-302. doi:10.1111/j.1528-

1167.2012.03534.x.

26. Beniczky S, Lantz G, Rosenzweig I, Åkeson P, Pedersen B, Pinborg LH, Ziebell M, Jespersen B, Fuglsang-Frederiksen A. Source localization of rhythmic ictal EEG activity: a study of diagnostic accuracy following STARD criteria. Epilepsia. 2013 Oct;54(10):1743-52. doi: 10.1111/epi.12339.

27. Monnerat BZ, Velasco TR, Assirati JA Jr, Carlotti CG Jr, Sakamoto AC. On the prognostic value of ictal EEG patterns in temporal lobe epilepsy surgery: a cohort study. Seizure. 2013 May;22(4):287-91. doi: 10.1016/j.seizure.2013.01.019.

28. Wieser HG, Blume WT, Fish D, Goldensohn E, Hufnagel A, King D, Sperling MR, Lüders H, Pedley TA; Commission on Neurosurgery of the International League Against Epilepsy (ILAE). ILAE Commission Report. Proposal for a new classification of outcome with respect to epileptic seizures following epilepsy surgery. Epilepsia. $2001 \mathrm{Feb} ; 42(2): 282-6$

29. Jaeschke R, Guyatt GH, Sackett DL. Users' guides to the medical literature. III. How to use an article about a diagnostic test. B. What are the results and will they help me in caring for my patients? The Evidence-Based Medicine Working Group. JAMA. 1994 Mar 2;271(9):703-7

30. Youden WJ. Index for rating diagnostic tests. Cancer. $1950 \mathrm{Jan} ; 3(1): 32-5$. 
31. Zweig MH, Campbell G. Receiver-operating characteristic (ROC) plots: a fundamental evaluation tool in clinical medicine. Clin Chem. 1993 Apr;39(4):561-77

32. Hawass NE. Comparing the sensitivities and specificities of two diagnostic procedures performed on the same group of patients. Br J Radiol. 1997 Apr;70(832):360-6.

33. Landis JR, Koch GG. The measurement of observer agreement for categorical data. Biometrics. 1977 Mar;33(1):159-74.

34. Bossuyt PM, Reitsma JB, Bruns DE, Gatsonis CA, Glasziou PP, Irwig L, Lijmer JG, Moher D, Rennie D, de Vet HC, Kressel HY, Rifai N, Golub RM, Altman DG, Hooft L, Korevaar DA, Cohen JF; STARD Group. STARD 2015: An Updated List of Essential Items for Reporting Diagnostic Accuracy Studies. Clin Chem. 2015 Dec;61(12):144652. doi: $10.1373 /$ clinchem. 2015.246280 .

35. Biggerstaff BJ. Comparing diagnostic tests: a simple graphic using likelihood ratios. Stat Med. 2000 Mar 15;19(5):649-63.

36. Tong X, An D, McGonigal A, Park SP, Zhou D. Validation of the Generalized Anxiety Disorder-7 (GAD-7) among Chinese people with epilepsy. Epilepsy Res. 2015 Nov 28;120:31-36. doi: 10.1016/j.eplepsyres. 2015.11.019. 
37. Velasco TR, Wichert-Ana L, Leite JP, Araújo D, Terra-Bustamante VC, Alexandre V Jr, Kato M, Assirati JA Jr, Machado HR, Carlotti CG Jr, Sakamoto AC. Accuracy of ictal SPECT in mesial temporal lobe epilepsy with bilateral interictal spikes. Neurology. 2002 Jul 23;59(2):266-71.

38. Patil SG, Cross JH, Kling Chong W, Boyd SG, Harkness WJ, Neville BG, Scott RC. Is streamlined evaluation of children for epilepsy surgery possible? Epilepsia. 2008 Aug;49(8):1340-7. doi: 10.1111/j.1528-1167.2008.01538.x.

39. Cendes F, Li LM, Watson C, Andermann F, Dubeau F, Arnold DL. Is ictal recording mandatory in temporal lobe epilepsy? Not when the interictal electroencepalogram and hippocampal atrophy coincide. Arch Neurol. 2000 Apr;57(4):497-500.

40. Morris HH, Matkovic Z, Estes ML, Prayson RA, Comair YG, Turnbull J, Najm I,Kotagal P, Wyllie E. Ganglioglioma and intractable epilepsy: clinical and neurophysiologic features and predictors of outcome after surgery. Epilepsia. 1998 Mar;39(3):307-13.

41. Wyllie E, Lachhwani DK, Gupta A, Chirla A, Cosmo G, Worley S, Kotagal P, Ruggieri P, Bingaman WE. Successful surgery for epilepsy due to early brain lesions despite generalized EEG findings. Neurology. 2007 Jul 24;69(4):389-97. 
42. Holmes MD, Wilensky AJ, Ojemann GA, Ojemann LM. Hippocampal or neocortical lesions on magnetic resonance imaging do not necessarily indicate site of ictal onsets in partial epilepsy. Ann Neurol. 1999 Apr;45(4):461-5.

43. Dash GK, Radhakrishnan A, Kesavadas C, Abraham M, Sarma PS, Radhakrishnan K. An audit of the presurgical evaluation and patient selection for extratemporal resective epilepsy surgery in a resource-poor country. Seizure. 2012 Jun;21(5):361-6. doi: 10.1016/j.seizure.2012.03.005. 
Table 01

\begin{tabular}{|c|c|}
\hline Female; N (\%) & $25(58.1)$ \\
\hline Mean age at LTM; years (range) & $17.17(1-58)$ \\
\hline Mean epilepsy duration at LTM; years (range) & $11.79(0-49)$ \\
\hline Mean age at surgery; years (range) & $18.70(2-58)$ \\
\hline Mean epilepsy duration at surgery; years (range) & $13.44(1-49)$ \\
\hline Mean follow-up post-surgery; years (range) & $6.4(1-16)$ \\
\hline $1.5 \mathrm{~T}$ MRI; $\mathrm{N}(\%)$ & $32(74.4)$ \\
\hline Availability of routine EEG; N (\%) & $14(32.6)$ \\
\hline \multicolumn{2}{|l|}{ Invasive studies performed; N (\%) } \\
\hline Acute only & $21(48.8)$ \\
\hline Chronic & $11(25.6)$ \\
\hline \multicolumn{2}{|l|}{ Cortical stimulation performed; $\mathrm{N}(\%)$} \\
\hline Acute only & $8(18.6)$ \\
\hline Chronic & $5(11.6)$ \\
\hline Seizure-free at last appointment; N (\%) & $24(55.8)$ \\
\hline
\end{tabular}

Table 02

\begin{tabular}{|c|c|c|c|c|}
\hline \multicolumn{5}{|l|}{ a) LTM } \\
\hline & & Seizure-free & Not seizure-free & \\
\hline & Concordant & 19 (TP) & $12(\mathrm{FP})$ & 31 \\
\hline & Discordant & $3(\mathrm{FN})$ & 7 (TN) & 10 \\
\hline & & 22 & 19 & 41 \\
\hline \multicolumn{5}{|l|}{ b) MRI } \\
\hline & & Seizure-free & Not seizure-free & \\
\hline & $\begin{array}{l}\text { Concordant } \\
\end{array}$ & 23 (TP) & $13(\mathrm{FP})$ & 36 \\
\hline & Discordant & $1(\mathrm{FN})$ & 6 (TN) & 7 \\
\hline & & 24 & 19 & 43 \\
\hline
\end{tabular}


Table 03

\begin{tabular}{|c|c|c|}
\hline & LTM & MRI \\
\hline Sensitivity, $\%(95 \% \mathrm{CI})$ & $86.3(64-96.4)$ & $95.8(76.8-99.7)$ \\
\hline Specificity, \% (95\% Cl) & $36.8(17.2-61.3)$ & $31.5(13.5-56.5)$ \\
\hline Positive Predictive Value, \% (95\% CI) & $61.2(42.2-77.5)$ & $63.8(46.2-78.6)$ \\
\hline Negative Predictive Value, $\%(95 \% \mathrm{Cl})$ & $70(35.3-91.9)$ & $85.7(42-99.2)$ \\
\hline Positive Likelihood Ratio (95\% CI) & $1.360(0.93-2)$ & $1.4(1.02-1.92)$ \\
\hline Negative Likelihood Ratio $(95 \% \mathrm{Cl})$ & $0.370(0.108-1.264)$ & $0.131(0.015-1.123)$ \\
\hline $\mathrm{AUC}_{\mathrm{ROC}}$ & $0.580(0.405-0.755)$ & $0.637(0.464-0.810)$ \\
\hline Youden's index & $0.232(0.102-0.362)$ & $0.274(0.165-0.383)$ \\
\hline
\end{tabular}

All differences non-significant $(P>0.05)$ 
Figure 01

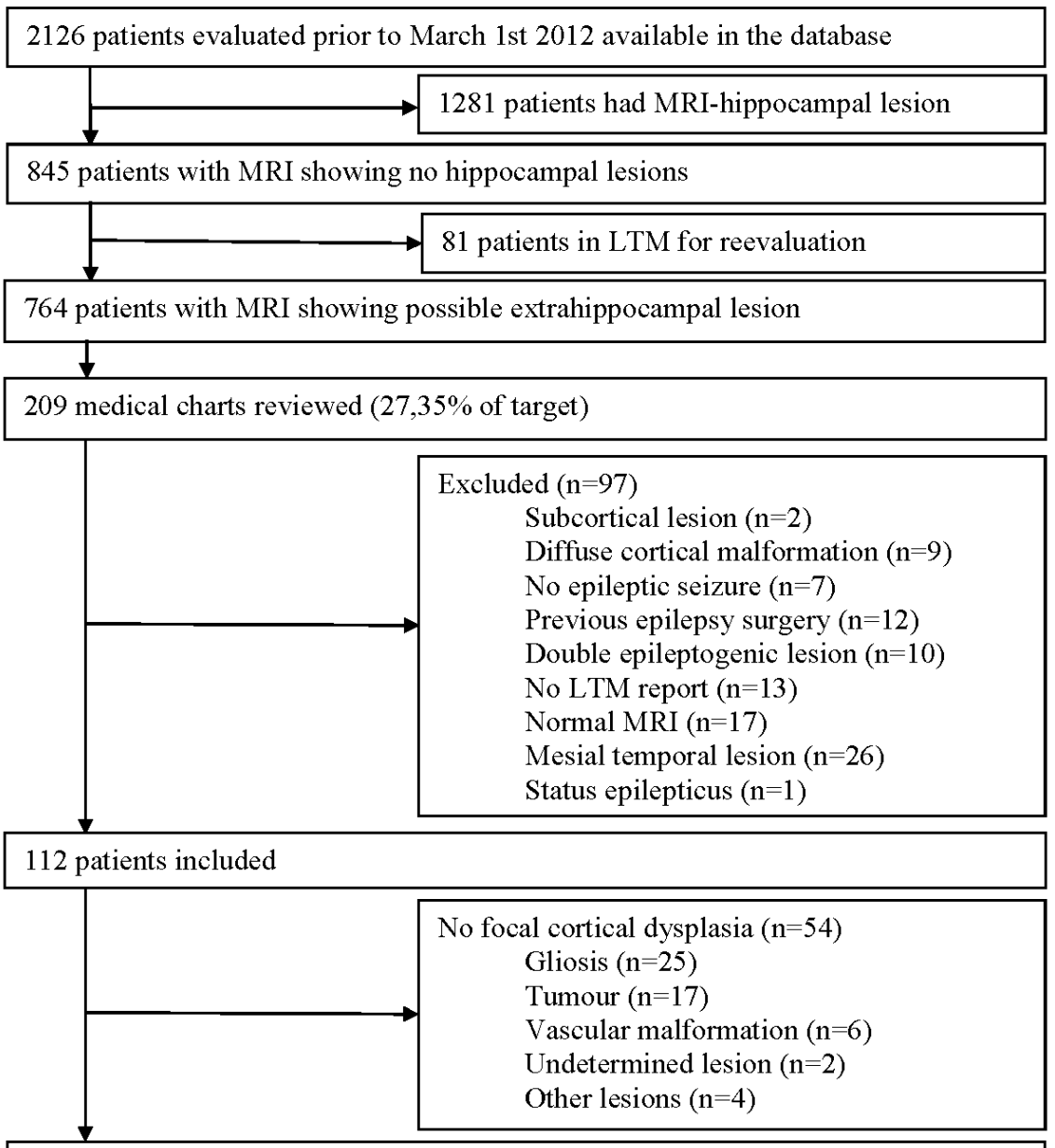

58 patients with focal cortical dysplasia selected

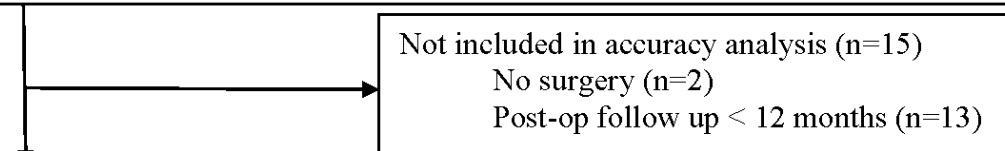

43 patients were eligible for accuracy analysis.

Figure 1. Patient selection. LTM: long-term video-EEG monitoring. MRI: magnetic resonance imaging of the brain. 


\section{Figure 02}

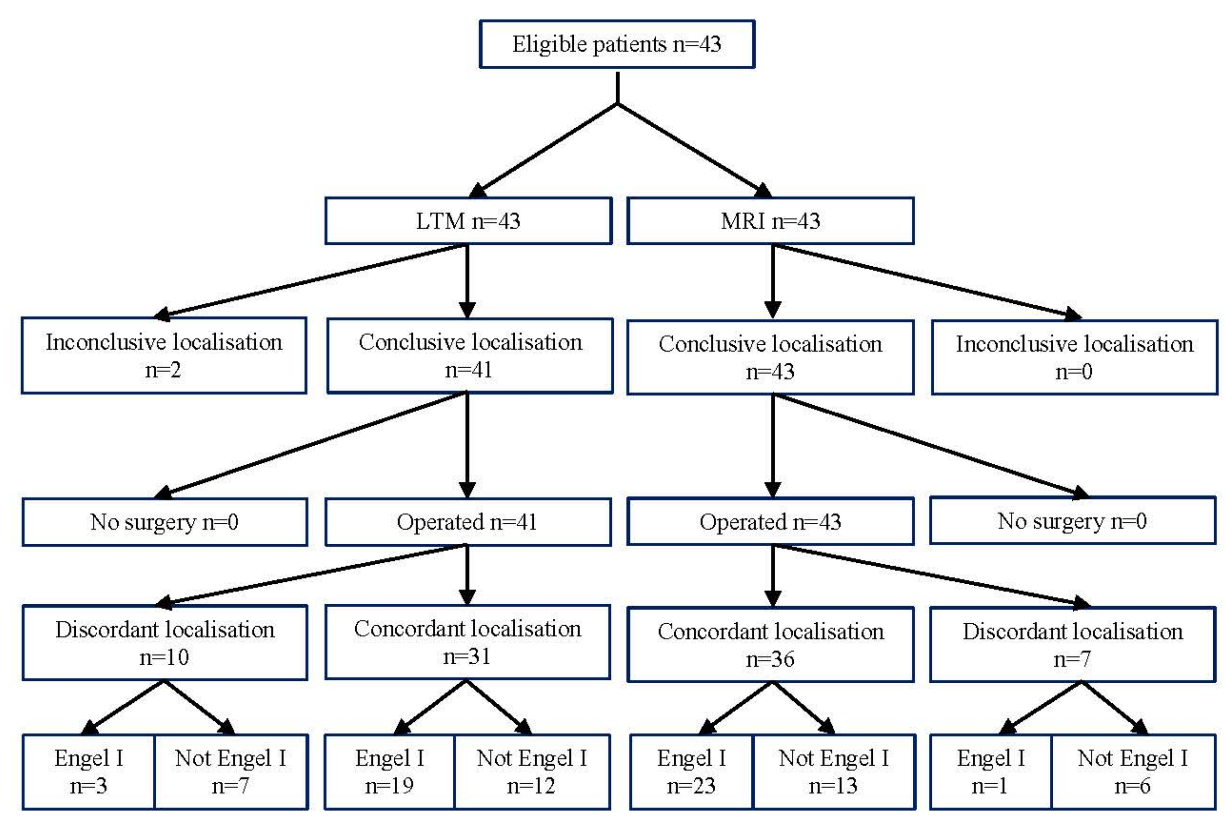

Figure 2. Flowchart of results. LTM: long-term video-EEG monitoring. MRI: magnetic resonance imaging of the brain. Gold-standard: region of surgery (at lobar level) in seizure-free patients.

Concordant localisation: match between the test and the gold-standard. Discordant localisation: no match between the test and the gold-standard. 
Figure 03

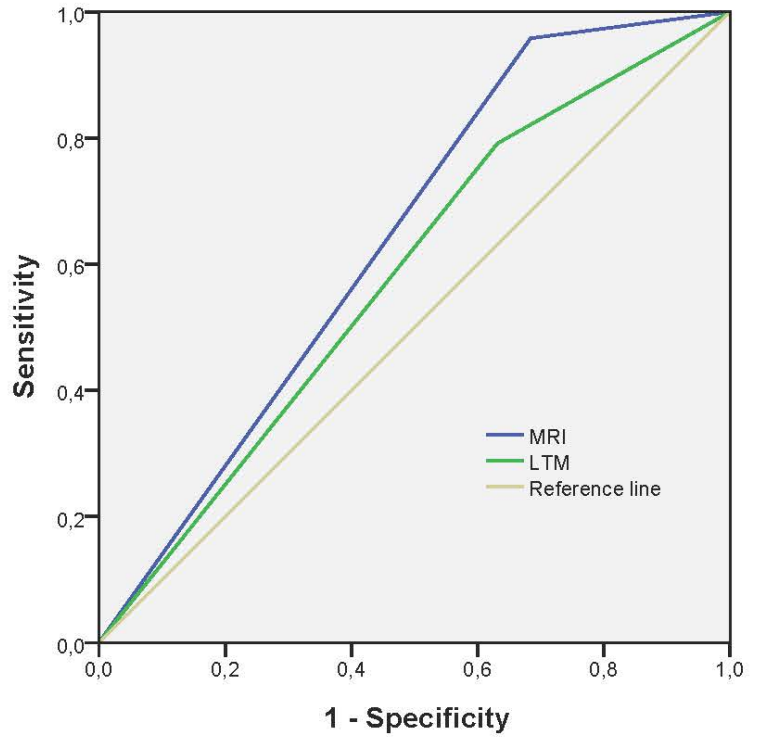

Figure 3. Receiver-operating characteristic (ROC) curves. LTM: Iong-term video-EEG monitoring. MRI: magnetic resonance imaging of the brain. 

Anexos 

ANEXO A - Aprovação do Comitê de Ética em Pesquisa (CEP). 


\section{HOSPITAL DAS CLÍNICAS DA
FACULDADE DE MEDICINA DE Platoformo
RIBEIRÃO PRETO DA USP -}

\section{PARECER CONSUBSTANCIADO DO CEP}

\section{DADOS DO PROJETO DE PESQUISA}

Título da Pesquisa: Convergência da videoeletroencefalografia prolongada e da ressonância magnética de encéfalo na determinação de zonas epileptogênicas extrahipocampais presumidas.

Pesquisador: Bruno Zanotelli Monnerat

Área Temática:

Versão: 2

CAAE: 45858415.0 .0000 .5440

Instituição Proponente: Hospital das Clínicas da Faculdade de Medicina de Ribeirão Preto da USP -

Patrocinador Principal: Financiamento Próprio

\section{DADOS DO PARECER}

Número do Parecer: 1.335 .345

\section{Apresentação do Projeto:}

A epilepsia é uma doença comum, que implica em alta morbidade. É intratável com medicação em cerca de $35 \%$ dos acometidos, e nestes casos é necessário que se estude a possivel cura por meio da ressecção de regiões do cérebro envolvidas na doença. Nos casos em que existe lesão cerebral epileptogênica identificada à ressonância magnética de encéfalo IRM, uma chance superior de vida livre de crises ocorre naqueles pacientes cuja lesão é ressecada. Atualmente, é habitual que sejam registradas crises epilépticas por intermédio de videoeletroencefalografia prolongada VEEG para que seja documentada a convergência do quadro clínico com a lesão cerebral, de forma que a decisão de prosseguir com cirurgia possa ser tomada. Este estudo questiona se é necessário tal método diagnóstico para a tomada de decisão cirúrgica, tendo em perspectiva o tipo de lesão epileptogênica.

\section{Objetivo da Pesquisa:}

Objetivo Primário: Determinar se o VEEG pode ser dispensado na tomada de decisão e planejamento cirúrgico no tratamento de epilepsias focais extrahipocampais lesionais

Objetivo Secundário: Determinar quais variáveis podem contribuir para a convergência de resultados da videoeletroencefalografia e da ressonância magnética nos pacientes candidatos à cirurgia de epilepsia.

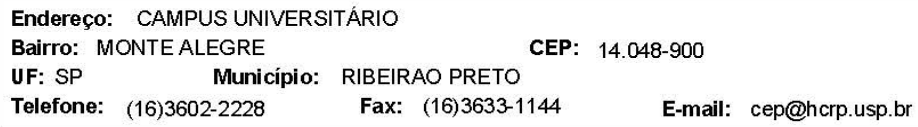




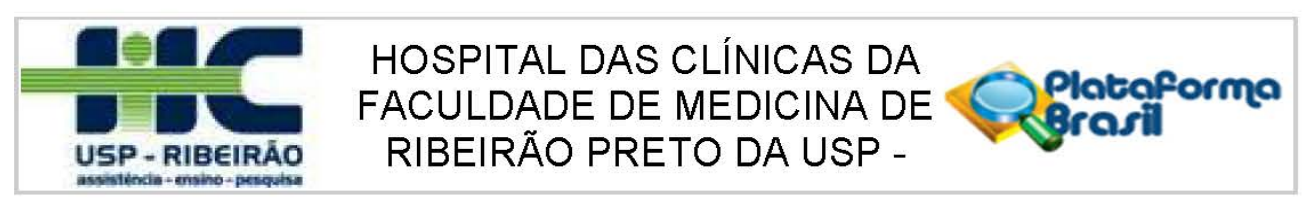

Cantinuaçä́ do Parecer: 1.335 .345

\section{Avaliação dos Riscos e Benefícios:}

Riscos: O presente trabalho implica em risco para os sujeitos da pesquisa, uma vez que, embora se trate de estudo retrospectivo, os dados referentes aos sujeitos da pesquisa podem ser acessados por indivíduos estranhos ao projeto. Para minimizar este risco, apenas o pesquisador e seu orientador terão acesso aos dados durante a execução do estudo. Isto visa à preservação do sigilo e confidencialidade das informações. Benefícios: Determinação se um exame dispendioso pode ser subtraído da avaliação cirúrgica de epilepsia, sem prejuízo para a tomada de decisão sobre o local da operação.

Comentários e Considerações sobre a Pesquisa:

Projeto em concordância com os princípios éticos preconizados.

\section{Considerações sobre os Termos de apresentação obrigatória:}

o pesquisador encaminhou a versão 2 do projeto, corrigido conforme solicitação do parecer emitido em 14/10/2015.

\section{Recomendações:}

Conclusões ou Pendências e Lista de Inadequações:

Diante do exposto e à luz da Resolução CNS 466/2012, o projeto de pesquisa versão 2 de 07/11/2015, assim como a dispensa do Termo de Consentimento Livre e Esclarecido, podem ser enquadrados na categoria APROVADO.

\section{Considerações Finais a critério do CEP:}

Projeto Aprovado: Tendo em vista a legislação vigente, devem ser encaminhados ao CEP, relatórios parciais anuais referentes ao andamento da pesquisa e relatório final ao término do trabalho. Qualquer modificação do projeto original deve ser apresentada a este CEP em nova versão, de forma objetiva e com justificativas, para nova apreciação.

Este parecer foi elaborado baseado nos documentos abaixo relacionados:

\begin{tabular}{|l|l|c|l|c|}
\hline \multicolumn{1}{|c|}{ Tipo Documento } & \multicolumn{1}{|c|}{ Arquivo } & Postagem & \multicolumn{1}{c|}{ Autor } & Situação \\
\hline $\begin{array}{l}\text { Informações Básicas } \\
\text { do Projeto }\end{array}$ & $\begin{array}{l}\text { PB_INFORMAÇŌES_BÁSICAS_DO_P } \\
\text { ROJETO 524952.pdf }\end{array}$ & $\begin{array}{c}07 / 11 / 2015 \\
16: 19: 29\end{array}$ & & Aceito \\
\hline $\begin{array}{l}\text { Declaração de } \\
\text { Pesquisadores }\end{array}$ & Carta_CEP.pdf & $\begin{array}{c}07 / 11 / 2015 \\
16: 18: 09\end{array}$ & $\begin{array}{l}\text { Bruno Zanotelli } \\
\text { Monnerat }\end{array}$ & Aceito \\
\hline $\begin{array}{l}\text { Projeto Detalhado/ } \\
\text { Brochura } \\
\text { Investigador }\end{array}$ & Projeto_Convergencia_v2.pdf & $\begin{array}{l}07 / 11 / 2015 \\
16: 17: 03\end{array}$ & $\begin{array}{l}\text { Bruno Zanotelli } \\
\text { Monnerat }\end{array}$ & Aceito \\
\hline
\end{tabular}

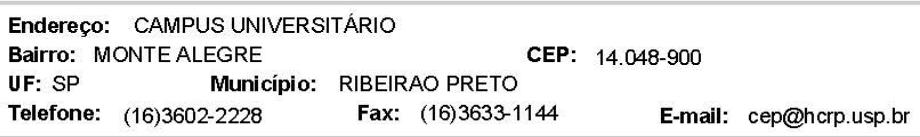




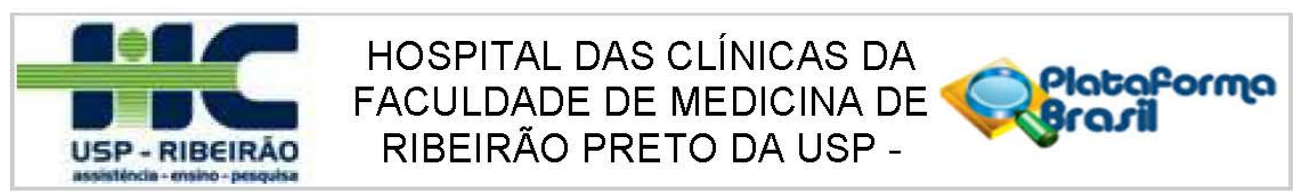

Continuaçẩo do Parecer: 1.335 .345

\begin{tabular}{|c|c|c|c|c|}
\hline Outros & DispensaTCLE.pdf & $\begin{array}{c}14 / 10 / 2015 \\
08: 18: 44\end{array}$ & $\begin{array}{l}\text { Bruno Zanotelli } \\
\text { Monnerat }\end{array}$ & Aceito \\
\hline Orçamento & OrcamentoUPCassinado.pdf & $\begin{array}{c}14 / 10 / 2015 \\
08: 17: 45\end{array}$ & $\begin{array}{l}\text { Bruno Zanotelli } \\
\text { Monnerat }\end{array}$ & Aceito \\
\hline Folha de Rosto & folhaDeRosto.pdf & $\begin{array}{c}14 / 10 / 2015 \\
08: 15: 55\end{array}$ & $\begin{array}{l}\text { Bruno Zanotelli } \\
\text { Monnerat }\end{array}$ & Aceito \\
\hline
\end{tabular}

Situação do Parecer:

Aprovado

Necessita Apreciação da CONEP:

Não

RIBEIRAO PRETO, 24 de Novembro de 2015

Assinado por:

MARCIA GUIMARÃES VILLANOVA

(Coordenador)

Endereço: CAMPUS UNIVERSITÁRIO

Bairro: MONTE ALEGRE

UF: SP Município: RIBEIRAO PRETO

Telefone: (16)3602-2228 Fax: (16)3633-1144 E-mail: cep@hcrp.usp.br 